\title{
Visible Light Stimulated Bistable Photo-Switching in Defect Engineered Metal-Organic Frameworks
}

Nicholas D. Shepherd, ${ }^{\dagger}$ Tiesheng Wang, ${ }^{\dagger \dagger}$ Bowen Ding, ${ }^{\dagger}$ Jonathon E. Beves, ${ }^{\S}$ and Deanna M. $\mathrm{D}^{\prime}$ Alessandro ${ }^{\dagger *}$

† School of Chemistry, The University of Sydney, Sydney, NSW 2006, Australia.

School of Mechanical Engineering, Shanghai Jiao Tong University, Shanghai 200240, China.

$\S$ School of Chemistry, The University of New South Wales, Sydney, NSW 2052, Australia.

*Corresponding author: deanna.dalessandro@sydney.edu.au

\section{Contents}




\section{S1. Experimental}

\section{General Characterisation}

PXRD patterns were obtained in flat-plate mode on a PANalytical Expert Pro MPD Powder XRay Diffractometer employing $\mathrm{Cu} \mathrm{K} \alpha$ radiation $(\lambda=1.5406 \AA)$. The diffractometer was fitted with a PIXcel detector $\left(45 \mathrm{kV}, 40 \mathrm{~mA}, 1^{\circ}\right.$ divergence/anti-scatter slits and $0.3 \mathrm{~mm}$ detector/receiver slits). Pawley extractions were conducted using GSAS2. ${ }^{1}$

Solution-state ${ }^{1} \mathrm{H}$ NMR spectra were recorded on a Bruker AVANCEIII $300 \mathrm{MHz}$ spectrometer

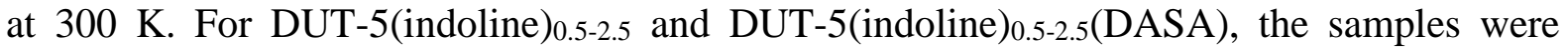
digested in DMSO- $d_{6}$ and TFA. Chemical shifts $(\delta)$ were calibrated against solvent residual signals. ${ }^{2}$ Positive and negative ion ESI-MS spectra were obtained using a Bruker amaZon SL mass spectrometer. HPLC grade $\mathrm{MeOH}$ was used for serial 10× dilutions. ATR-FTIR spectra were recorded on PerkinElmer Spectrum Two IR spectrometer with UATR attachment. Spectra were recorded with 32 scans at $2 \mathrm{~cm}^{-1}$ resolution. TGA data was obtained using a Discovery TGA Thermogravimetric Analyser. Measurements involved ramping the temperature at $2{ }^{\circ} \mathrm{C}$ $\min ^{-1}$ to $700{ }^{\circ} \mathrm{C}$ under compressed air at a $20 \mathrm{~mL} / \mathrm{min}$ flow rate. Elemental analyses were conducted using a Vario MICRO cube elemental analyser (Elementar Analysensysteme $\mathrm{GmbH}$, Germany). Samples were dried at $110^{\circ} \mathrm{C}$ prior to analysis.

Reaction kinetic studies for DASAIND syntheses were performed on a CARY5000 UV-vis spectrophotometer. $\mathrm{Me}_{2} \mathrm{DP}(\approx 2.0 \mathrm{mg}, 8.5 \mathrm{mmol})$ was dissolved in THF $(10.0 \mathrm{~mL})$ and diluted by a factor of five. Indoline ( $\approx 2.0 \mathrm{mg}, 9.8 \mathrm{mmol})$ was added to the solution in the cuvette before three drops of $\mathrm{Et}_{3} \mathrm{~N}$ and scans were commenced. THF was recorded for a baseline spectrum. Spectra were recorded over a 10000-40000 $\mathrm{cm}^{-1}$ range at $6000 \mathrm{~cm}^{-1} \mathrm{~min}^{-1}$. Measurements were taken at 30 minute intervals over a 20 hour period from addition of the activated furan solution.

Solid-state diffuse reflectance UV-vis measurements were carried out using a Cary5000 spectrophotometer. Scans were collected at a rate of $6000 \mathrm{~cm}^{-1} \mathrm{~min}^{-1}$ over a range of 10000$40000 \mathrm{~cm}^{-1}$ for initial solid-state spectra. Additional spectra were collected following irradiation with white LED lights arranged on a breadboard circuit at 20 minute intervals within the range of $10000-30000 \mathrm{~cm}^{-1}$. Irradiated samples were immediately subject to VT UV-vis where the same instrument and run parameters were used. The temperature was controlled 
using a Harrick VT cell, with spectra being recorded at $10{ }^{\circ} \mathrm{C}$ intervals. For long-term stability measurements, an Omni-Diff probe was used, with a scan range of $10000-30000 \mathrm{~cm}^{-1}$. For stability testing, spectra were checked over three cycles with an Omni-Diff probe using the same parameters as previously described prior to PXRD analysis conducted as previously described. Diffuse reflectance data were converted to Kubelka Munk units $F(R)=\left(1-R^{2}\right) / 2 R$ for representation.

\section{Gas Sorption}

$\mathrm{N}_{2}(77 \mathrm{~K})$ and $\mathrm{CO}_{2}(298 \mathrm{~K})$ isotherms were recorded on a 3Flex Surface Characterisation Analyser (Micromeritics Instruments Inc.). Prior to this, samples were outgassed under dynamic vacuum $\left(\sim 10^{-6}\right.$ bar) at either $130{ }^{\circ} \mathrm{C}$ (DUT-5 and DUT-5(indoline) $\left.0.5-2.5\right)$ or $60{ }^{\circ} \mathrm{C}$ (DUT-5(indoline) 0.5-2.5(DASA)) for 20 hours. The $\mathrm{S}_{\mathrm{BET}}$ for all frameworks were calculated with BET theory. ${ }^{3}$ Pore size distribution was calculated with DFT (NLDFT, based on $\mathrm{N}_{2}$ Cylindrical Pores-Oxide Surface model). These calculations were conducted in the 3Flex software (version 4.01, Micromeritics Instrument Corporation). For DUT-5(indoline) $)_{0.5-2.5}(\mathrm{DASA})$, initial $\mathrm{CO}_{2}$ isotherms were recorded before and after irradiating with white light for three hours. Stability following gas sorption analysis was confirmed through PXRD analysis conducted as previously described.

\section{Materials \& Syntheses}

All chemical reagents were used as provided by commercial suppliers without additional purification.

\section{2,3,3-Trimethyl-3H-indole-5-carboxylic acid (indoline)}

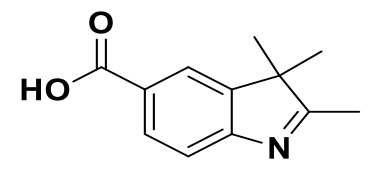

4-Hydrazinobenzoic acid (2.1 g, $14 \mathrm{mmol})$, glacial acetic acid (15 mL) and 3-methyl-2butanone $(2.6 \mathrm{~mL}, 24 \mathrm{mmol})$ were added to a $30 \mathrm{~mL}$ reaction vial. The solution was heated in an Anton Paar Monowave 300 microwave at $160{ }^{\circ} \mathrm{C}$ for an hour with magnetic stirring (600 $\mathrm{rpm})$. After cooling to room temperature, the solution was brought to approximately $\mathrm{pH} 13.0$ 
with an aqueous $\mathrm{KOH}$ solution. The $\mathrm{pH}$ was adjusted to 4.0 with $\mathrm{HCl}(32 \%)$ and allowed to stand. The solution was filtered and the brown precipitate (indoline) was washed with $\mathrm{H}_{2} \mathrm{O}$ and dried in vacuo. Yield: $1.8 \mathrm{~g}(65 \%)$.

${ }^{1} \mathrm{H}$ NMR $\left(300 \mathrm{MHz}, \mathrm{CDCl}_{3}\right) \delta: 8.14(\mathrm{~d}, J=0.01,1 \mathrm{H}, \mathrm{Ar}-\mathrm{H}), 8.06(\mathrm{~s}, 1 \mathrm{H}, \mathrm{Ar}-\mathrm{H}), 7.66(\mathrm{~d}, J=$ 0.02, 1H, Ar-H), 2.37 (s, 3H, C=C-C-H) and $1.37\left(\mathrm{~s}, 6 \mathrm{H}, \mathrm{C}-\left(\mathrm{CH}_{3}\right)_{2}\right)$. (+)ESI-MS $(\mathrm{MeOH})$ found $m / z$ 204.1 (calc. $[\mathrm{M}+1]^{+} \mathrm{m} / \mathrm{z}$ 204.1). (-)ESI-MS (MeOH) found $m / z 202.2$ (calc. $[\mathrm{M}-1]^{-} \mathrm{m} / \mathrm{z}$ 202.1). ATR-FTIR (cm-1): 1110 (m), 1212 (s), 1230 (vs), 1296 (s), 1385 (w), 1421 (m), 1464 (w), $1570(\mathrm{~m}), 1616(\mathrm{w})$ and 1680 (s). Elemental analysis $\left(\mathrm{C}_{12} \mathrm{H}_{13} \mathrm{NO}_{2}\right)$ : C (calc. 70.92, found 68.58), $\mathrm{H}$ (calc. 6.45, found 6.37) and $\mathrm{N}$ (calc. 6.89, found 6.60).

\section{5-(Furan-2-ylmethylene)-1,3-dimethylpyrimidine-2,4,6(1H,3H,5H)-trione $\left(\mathrm{Me}_{2} \mathrm{DP}\right)$}<smiles>CN1C(=O)C(=Cc2ccco2)C(=O)N(C)C1=O</smiles>

$\mathrm{Me}_{2} \mathrm{DP}$ was prepared using a literature procedure. ${ }^{4,5}$ 1,3-Dimethylbarbituric acid (2.97 g, 19.1 mmol) was dissolved in $\mathrm{H}_{2} \mathrm{O}(50 \mathrm{~mL})$. Furfural $(2.0 \mathrm{~mL}, 24 \mathrm{mmol})$ was added dropwise, prompting formation of a yellow precipitate. The solution was stirred for two hours and filtered. The yellow solid ( $\left.\mathrm{Me}_{2} \mathrm{DP}\right)$ was dried in vacuo. Yield: $4.145 \mathrm{~g}(93 \%)$.

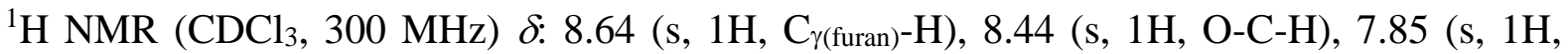
$\left.\mathrm{C}_{\beta(\text { furan) }}-\mathrm{H}\right), 6.74(\mathrm{~s}, 1 \mathrm{H}, \mathrm{C}=\mathrm{C}-\mathrm{H})$ and $3.41\left(\mathrm{~s}, 6 \mathrm{H},\left(\mathrm{N}-\mathrm{CH}_{3}\right)_{2}\right) .(+) \mathrm{ESI}-\mathrm{MS}(\mathrm{MeOH})$ found $\mathrm{m} / \mathrm{z}$ 257.1 (calc. $\left.\left[\mathrm{M}+\mathrm{Na}^{+}\right]^{+} \mathrm{m} / z, 257.1\right)$. ATR-FTIR $\left(\mathrm{cm}^{-1}\right): 1022$ (m), 1086 (m), 1159 (m), 1199 (m), $1255(\mathrm{~m}), 1280(\mathrm{~m}), 1361$ (s), $1411(\mathrm{~m}), 1460(\mathrm{~m}), 1575(\mathrm{~m})$ and $1652(\mathrm{~s})$.

1-((1E,3Z)-5-(1,3-dimethyl-2,4,6-trioxotetrahydropyrimidin-5(2H)-ylidene)-4-

hydroxypenta-1,3-dien-1-yl)-3,3-dimethyl-2-methyleneindoline-5-carboxylic

acid (DASAIND)<smiles>C=C1N(/C=C/C=C(\O)C=C2C(=O)N(C)C(=O)N(C)C2=O)c2ccc(C(=O)O)cc2C1(C)C</smiles> 
$\mathrm{Me}_{2} \mathrm{DP}$ (198.8 mg, $\left.0.8488 \mathrm{mmol}\right)$ was dissolved in THF (10 mL) where indoline (176.6 mg, $0.8689 \mathrm{mmol}$ ) was added to the solution. Et $3 \mathrm{~N}$ (5 drops) was added and the solution was stirred at room temperature for two days. Product was cooled in an $\mathrm{NaCl} /$ ice bath for 20 minutes. The product was crashed out with cold $\mathrm{Et}_{2} \mathrm{O}$ and filtered for a purple solid (DASA IND) that went green upon drying. Yield: $26.4 \mathrm{~g}(13 \%)$.

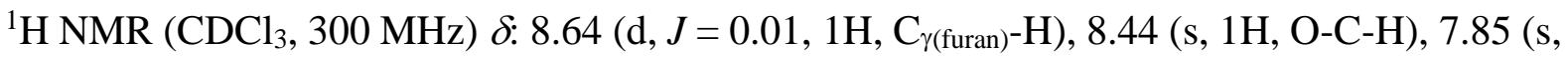
$\left.1 \mathrm{H}, \mathrm{C}_{\beta \text { (furan) }}-\mathrm{H}\right), 6.74(\mathrm{~d}, J=0.01,1 \mathrm{H}, \mathrm{C}=\mathrm{C}-\mathrm{H})$ and $3.40\left(\mathrm{~s}, 6 \mathrm{H},\left(\mathrm{N}-\mathrm{CH}_{3}\right)_{2}\right) .(+) \mathrm{ESI}-\mathrm{MS}(\mathrm{MeOH})$ found $m / z 239.2$ (calc. $[\mathrm{M}+1]^{+} \mathrm{m} / \mathrm{z}$ 438.2). ATR-FTIR $\left(\mathrm{cm}^{-1}\right)$ : $1035(\mathrm{~m}), 1074(\mathrm{~m}), 1126(\mathrm{~m})$, 1236 (m), 1261 (m), 1289 (m), 1374 (s), 1433 (s), 1577 (s), 1671 (s), 2326 (vw), 2358 (vw) and 2957 (vw).

\section{$[\mathrm{Al}(\mathrm{OH})(\mathrm{bpdc})](\mathrm{DMF})_{x}\left(\mathrm{H}_{2} \mathrm{O}\right)_{y}{ }^{z+}(\mathrm{DUT}-5)$}

$\mathrm{Al}\left(\mathrm{NO}_{3}\right)_{3} .9 \mathrm{H}_{2} \mathrm{O}(520 \mathrm{mg}, 1.39 \mathrm{mmol})$ and $\mathrm{H}_{2} \mathrm{bpdc}(261 \mathrm{mg}, 1.08 \mathrm{mmol})$ were added to a Parr pressure vessel and dissolved in DMF (30 mL). The solution was sealed and heated to $120{ }^{\circ} \mathrm{C}$ at $1{ }^{\circ} \mathrm{C} \mathrm{min}{ }^{-1}$. The temperature was maintained for one day before being allowed to cool to room temperature. The white powder (DUT-5) was separated by centrifugation, washed with water and dried in vacuo. Yield: $635 \mathrm{mg}(94 \%)$.

${ }^{1} \mathrm{H}$ NMR (300 MHz, digested in DMSO- $\left.d_{6} / \mathrm{TFA}\right) \delta: 8.04(\mathrm{~d}, J=0.03,4 \mathrm{H}$, Ar-H) and $7.85(\mathrm{~d}$, $J=0.03,4 \mathrm{H}, \mathrm{Ar}-\mathrm{H})$. ATR-FTIR $\left(\mathrm{cm}^{-1}\right): 539$ (s), 659 (w), 687 (m), 777 (s), 859 (w), 987 (w), $1093(\mathrm{w}), 1432(\mathrm{~s}), 1500(\mathrm{w}), 1551(\mathrm{w}), 1607$ (s) and $1669(\mathrm{~m})$.

\section{$\left[\mathrm{Al}(\mathrm{OH})(\mathrm{bpdc})_{\mathrm{x}}\left(\mathrm{C}_{12} \mathrm{H}_{12} \mathrm{NO}_{2}\right)_{\mathrm{x}}\right](\mathrm{DMF})_{x}\left(\mathrm{H}_{2} \mathrm{O}\right)_{y}{ }^{z+}\left(\mathrm{DUT}-5(\text { indoline })_{0.5-2.5}\right)$}

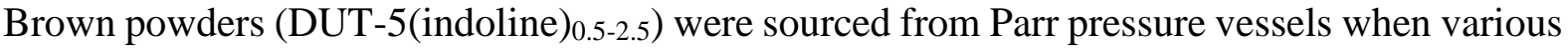
indoline quantities were added to the solvothermal synthesis described for DUT-5.

DUT-5(indoline) 0.5 was prepared with indoline $(101 \mathrm{mg}, 0.496 \mathrm{mmol}) .{ }^{1} \mathrm{H} \mathrm{NMR}(300 \mathrm{MHz}$, digested in DMSO- $\left.d_{6} / \mathrm{TFA}\right) \delta: 8.111(\mathrm{~s}, 4 \mathrm{H}, \mathrm{Ar}-\mathrm{H}), 8.01(\mathrm{~s}, 1 \mathrm{H}, \mathrm{Ar}-\mathrm{H}), 7.93(\mathrm{~s}, 4 \mathrm{H}, \mathrm{Ar}-\mathrm{H})$, $3.00\left(\mathrm{~s}, 3 \mathrm{H}, \mathrm{CH}_{3}\right)$ and $2.79\left(\mathrm{~s}, 6 \mathrm{H},\left(\mathrm{CH}_{3}\right)_{2}\right)$. ATR-FTIR ( $\left.\mathrm{cm}^{-1}\right): 539(\mathrm{~m}), 659(\mathrm{w}), 688(\mathrm{~m}), 777$ (s), $860(\mathrm{w}), 987(\mathrm{w}), 1093(\mathrm{w}), 1432(\mathrm{~s}), 1500(\mathrm{w}), 1551(\mathrm{w}), 1607$ (s) and $1669(\mathrm{~m})$.

DUT-5(indoline)1.0 was prepared with indoline (202 mg, $0.992 \mathrm{mmol}) .{ }^{1} \mathrm{H}$ NMR $(300 \mathrm{MHz}$, digested in DMSO- $d_{6} /$ TFA) $\delta: 8.05(\mathrm{~d}, J=0.02,4 \mathrm{H}, \mathrm{Ar}-\mathrm{H}), 7.94(\mathrm{~s}, 1 \mathrm{H}, \mathrm{Ar}-\mathrm{H}), 7.86(\mathrm{~d}, J=$ 0.03, 4H, Ar-H), $2.88\left(\mathrm{~s}, 3 \mathrm{H}, \mathrm{CH}_{3}\right)$ and $2.73\left(\mathrm{~s}, 6 \mathrm{H},\left(\mathrm{CH}_{3}\right)_{2}\right)$. ATR-FTIR $\left(\mathrm{cm}^{-1}\right): 541(\mathrm{~s}), 619$ 
(w), $659(\mathrm{w}), 688(\mathrm{~m}), 777(\mathrm{~s}), 860(\mathrm{w}), 987(\mathrm{w}), 1096(\mathrm{w}), 1433(\mathrm{~s}), 1501(\mathrm{w}), 1552(\mathrm{w}), 1608$ (s) and $1669(\mathrm{~m})$.

DUT-5(indoline)1.5 was prepared with indoline (306 mg, $1.50 \mathrm{mmol}) .{ }^{1} \mathrm{H}$ NMR $(300 \mathrm{MHz}$, digested in DMSO- $d_{6} /$ TFA) $\delta: 8.05(\mathrm{~d}, J=0.02,4 \mathrm{H}, \mathrm{Ar}-\mathrm{H}), 7.94(\mathrm{~s}, 1 \mathrm{H}, \mathrm{Ar}-\mathrm{H}), 7.85$ (d, $J=$ 0.02, 4H, Ar-H), $2.88\left(\mathrm{~s}, 3 \mathrm{H}, \mathrm{CH}_{3}\right)$ and $2.72\left(\mathrm{~s}, 6 \mathrm{H},\left(\mathrm{CH}_{3}\right)_{2}\right)$. ATR-FTIR $\left(\mathrm{cm}^{-1}\right): 540$ (s), 620 (w), $658(\mathrm{w}), 688(\mathrm{~m}), 776(\mathrm{~s}), 858(\mathrm{w}), 988(\mathrm{w}), 1097(\mathrm{w}), 1432(\mathrm{~s}), 1502(\mathrm{w}), 1552(\mathrm{w}), 1607$ (s) and $1669(\mathrm{~m})$.

DUT-5(indoline)2.0 was prepared with indoline (404 mg, $1.99 \mathrm{mmol}) .{ }^{1} \mathrm{H}$ NMR (300 MHz, digested in DMSO- $\left.d_{6} / \mathrm{TFA}\right) \delta: 8.04(\mathrm{~d}, J=0.03,4 \mathrm{H}, \mathrm{Ar}-\mathrm{H}), 7.93(\mathrm{~s}, 1 \mathrm{H}, \mathrm{Ar}-\mathrm{H}), 7.82(\mathrm{~d}, J=$ 0.03, 4H, Ar-H), $2.86\left(\mathrm{~s}, 3 \mathrm{H}, \mathrm{CH}_{3}\right)$ and $2.71\left(\mathrm{~s}, 6 \mathrm{H},\left(\mathrm{CH}_{3}\right)_{2}\right)$. ATR-FTIR $\left(\mathrm{cm}^{-1}\right): 537(\mathrm{~m}), 619$ (w), $659(\mathrm{w}), 687(\mathrm{~m}), 776(\mathrm{~s}), 858(\mathrm{w}), 987(\mathrm{w}), 1095(\mathrm{w}), 1429(\mathrm{~s}), 1501(\mathrm{w}), 1550(\mathrm{w}), 1605$ (s) and $1668(\mathrm{~m})$.

DUT-5(indoline) 2.5 was prepared with indoline (502 mg, $2.47 \mathrm{mmol}) .{ }^{1} \mathrm{H}$ NMR (300 MHz, digested in DMSO- $d_{6} /$ TFA) $\delta: 8.04(\mathrm{~d}, J=0.02,4 \mathrm{H}, \mathrm{Ar}-\mathrm{H}), 7.92(\mathrm{~s}, 1 \mathrm{H}, \mathrm{Ar}-\mathrm{H}), 7.80(\mathrm{~d}, J=$ 0.02, 4H, Ar-H), $2.85\left(\mathrm{~s}, 3 \mathrm{H}, \mathrm{CH}_{3}\right)$ and $2.71\left(\mathrm{~s}, 6 \mathrm{H},\left(\mathrm{CH}_{3}\right)_{2}\right)$. ATR-FTIR $\left(\mathrm{cm}^{-1}\right): 540(\mathrm{~m}), 620$ (w), $660(\mathrm{w}), 688(\mathrm{~m}), 776(\mathrm{~s}), 860(\mathrm{w}), 989(\mathrm{w}), 1096(\mathrm{w}), 1432(\mathrm{~s}), 1501(\mathrm{w}), 1552(\mathrm{w}), 1607$ (s) and $1670(\mathrm{~m})$.

$\left[\mathrm{Al}(\mathrm{OH})(\mathrm{bpdc})_{\mathrm{x}}\left(\mathrm{C}_{12} \mathrm{H}_{12} \mathrm{NO}_{2}\right)_{\mathrm{x}}(\mathrm{DASA})_{\mathrm{x}}\right](\mathrm{THF})_{x}{ }^{z+} \quad\left(\mathrm{DUT}-5(\text { indolinee })_{\text {0.5-2.5(DASA })) .}\right.$ DUT-

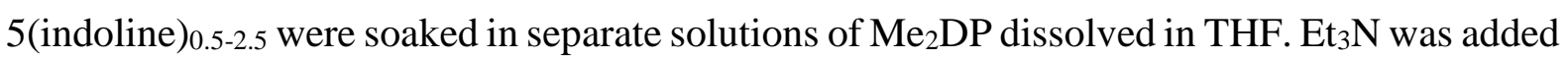
and the solutions were allowed to stand for six days. The purple solids (DUT-5(indoline)0.52.5(DASA)) were separated via centrifugation and washed with THF before air drying. Reagent/solvent quantities are summarised in Table S1.

DUT-5(indoline) 0.5 (DASA) ${ }^{1} \mathrm{H}$ NMR $\left(300 \mathrm{MHz}\right.$, digested in DMSO- $\left.d_{6} / \mathrm{TFA}\right) \delta: 8.05$ (d, $J=$ 0.03, 4H, Ar-H), 7.95 (s, 1H, Ar-H), 7.87 (d, $J=0.02,4 \mathrm{H}, \mathrm{Ar}-\mathrm{H}), 3.60$ (s, 1H, R $3 \mathrm{~N}^{-} \mathrm{H}^{+}$), 3.10 (t, $\left.J=0.02,1 \mathrm{H}, \mathrm{R}_{2} \mathrm{NH}^{+}-\mathrm{C}-\mathrm{H}\right), 2.89\left(\mathrm{~s}, 3 \mathrm{H}, \mathrm{N}-\mathrm{CH}_{3}\right), 2.73$ (s, 3H, N-CH$)_{3}$ and $1.76(\mathrm{~s}, 6 \mathrm{H}$, $\left.\left(\mathrm{CH}_{3}\right)_{2}\right)$. ATR-FTIR $\left(\mathrm{cm}^{-1}\right)$ : $542(\mathrm{~s}), 616(\mathrm{w}, \mathrm{sh}), 686(\mathrm{~m}), 775(\mathrm{~s}), 857(\mathrm{w}), 911(\mathrm{w}), 987(\mathrm{w})$, $1067(w), 1117(w), 1185(w), 1432$ (s), 1500 (w), 1552 (w), 1606 (s), 2865 (w) and $2974(w)$.

DUT-5(indoline)1.0(DASA) ${ }^{1} \mathrm{H}$ NMR $\left(300 \mathrm{MHz}\right.$, digested in DMSO- $\left.d_{6} / \mathrm{TFA}\right) \delta: 8.05$ (d, $J=$ 0.03, 4H, Ar-H), 7.95 (s, 1H, Ar-H), 7.87 (d, $J=0.02,4 \mathrm{H}, \mathrm{Ar}-\mathrm{H}), 3.10$ (t, $J=0.02,1 \mathrm{H}, \mathrm{R}_{2} \mathrm{NH}^{+}-$ $\mathrm{C}-\mathrm{H}), 2.89$ (s, 3H, N-CH 3$)$ and $2.73\left(\mathrm{~s}, 3 \mathrm{H}, \mathrm{N}-\mathrm{CH}_{3}\right)$. ATR-FTIR $\left(\mathrm{cm}^{-1}\right): 539$ (s), 616 (w, sh), 
$686(\mathrm{~m}), 775(\mathrm{~s}), 857(\mathrm{w}), 912(\mathrm{w}), 987(\mathrm{w}), 1066(\mathrm{w}), 1121(\mathrm{w}), 1185(\mathrm{w}), 1432(\mathrm{~s}), 1499(\mathrm{w})$, $1552(\mathrm{w}), 1606(\mathrm{~s}), 2872(\mathrm{w})$ and $2977(\mathrm{w})$.

DUT-5(indoline) 1.5(DASA) ${ }^{1} \mathrm{H}$ NMR $\left(300 \mathrm{MHz}\right.$, digested in DMSO- $\left.d_{6} / \mathrm{TFA}\right) \delta: 8.52(\mathrm{~s}, 1 \mathrm{H}$, Ar-H), 8.29 (s, 1H, Ar-H), 8.14 (s, 1H, Ar-H), 8.05 (d, J = 0.03, 4H, Ar-H), 7.95 (s, 1H, Ar$\mathrm{H}), 7.87(\mathrm{~d}, J=0.03,4 \mathrm{H}, \mathrm{Ar}-\mathrm{H}), 6.94(\mathrm{~s}, 1 \mathrm{H}, \mathrm{C}=\mathrm{C}-\mathrm{H}), 3.60\left(\mathrm{~s}, 1 \mathrm{H}, \mathrm{R}_{3} \mathrm{~N}-\mathrm{H}^{+}\right), 3.10(\mathrm{t}, J=0.02$, $\left.1 \mathrm{H}, \mathrm{R}_{2} \mathrm{NH}^{+}-\mathrm{C}-\mathrm{H}\right), 2.89\left(\mathrm{~s}, 3 \mathrm{H}, \mathrm{N}-\mathrm{CH}_{3}\right), 2.73\left(\mathrm{~s}, 3 \mathrm{H}, \mathrm{N}-\mathrm{CH}_{3}\right)$ and $1.75\left(\mathrm{~s}, 6 \mathrm{H},\left(\mathrm{CH}_{3}\right)_{2}\right)$. ATRFTIR ( $\left.\mathrm{cm}^{-1}\right)$ : 540 (s), 616 (w, sh), 686 (m), 776 (s), 857 (w), 912 (w), $988(w), 1066(w), 1121$ (w), $1185(w), 1434$ (s), 1506 (w), 1552 (w), 1607 (s), 2872 (w) and $2974(w)$.

DUT-5(indoline) 2.0(DASA) ${ }^{1} \mathrm{H}$ NMR $\left(300 \mathrm{MHz}\right.$, digested in DMSO- $\left.d_{6} / \mathrm{TFA}\right) \delta: 8.52(\mathrm{~s}, 1 \mathrm{H}$, Ar-H), 8.29 (s, 1H, Ar-H), 8.13 (s, 1H, Ar-H), 8.05 (d, $J=0.03,4 \mathrm{H}, \mathrm{Ar}-\mathrm{H}), 7.87$ (d, $J=0.03$, $4 \mathrm{H}, \mathrm{Ar}-\mathrm{H}), 6.94(\mathrm{~s}, 1 \mathrm{H}, \mathrm{C}=\mathrm{C}-\mathrm{H}), 3.10\left(\mathrm{t}, J=0.02,1 \mathrm{H}, \mathrm{R}_{2} \mathrm{NH}^{+}-\mathrm{C}-\mathrm{H}\right), 2.89\left(\mathrm{~s}, 3 \mathrm{H}, \mathrm{N}-\mathrm{CH}_{3}\right)$ and 2.73 (s, 3H, N-CH 3 ). ATR-FTIR ( $\left.\mathrm{cm}^{-1}\right): 540$ (s), 616 (w, sh), 686 (m), 775 (s), 856 (w), 909 (w), $986(\mathrm{w}), 1066(\mathrm{w}), 1120(\mathrm{w}), 1186(\mathrm{w}), 1432(\mathrm{~s}), 1499(\mathrm{w}), 1551(\mathrm{w}), 1606(\mathrm{~s}), 2871(\mathrm{w})$ and $2974(w)$.

DUT-5(indoline)2.5(DASA) ${ }^{1} \mathrm{H}$ NMR $\left(300 \mathrm{MHz}\right.$, digested in DMSO- $\left.d_{6} / \mathrm{TFA}\right) \delta: 8.51(\mathrm{~d}, J=$ $0.01,1 \mathrm{H}, \mathrm{Ar}-\mathrm{H}), 8.23$ (s, 1H, Ar-H), 8.13 (d, $J=0.03,1 \mathrm{H}, \mathrm{Ar}-\mathrm{H}), 8.04$ (d, $J=0.03,4 \mathrm{H}, \mathrm{Ar}-$ $\mathrm{H}), 7.83(\mathrm{~d}, J=0.03,4 \mathrm{H}, \mathrm{Ar}-\mathrm{H}), 6.89(\mathrm{~d}, J=0.02,1 \mathrm{H}, \mathrm{C}=\mathrm{C}-\mathrm{H})$ and $3.09(\mathrm{t}, J=0.01,1 \mathrm{H}$, R2 $\mathrm{NH}^{+}-\mathrm{C}-\mathrm{H}$ ). ATR-FTIR ( $\mathrm{cm}^{-1}$ ): 542 (s), 616 (w, sh), 687 (m), 776 (s), 857 (w), 910 (w), 988 (w), 1066 (w), 1185 (w), 1435 (s), 1508 (w), 1552 (w), 1608 (s), 2869 (w) and 2974 (w).

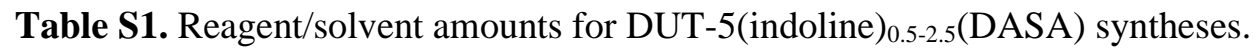

\begin{tabular}{lcccc}
\hline & DUT-5(indoline) $_{\mathrm{x}}$ & $\mathrm{THF}$ & $\mathrm{Me}_{2} \mathrm{DP}$ & $\mathrm{Et}_{3} \mathrm{~N}$ \\
\hline DUT-5(indoline) $)_{0.5}$ (DASA) & $100 \mathrm{mg}$ & $10 \mathrm{~mL}$ & $50 \mathrm{mg}$ & 10 drops \\
DUT-5(indoline) $)_{1.0}($ DASA) & $100 \mathrm{mg}$ & $10 \mathrm{~mL}$ & $100 \mathrm{mg}$ & 20 drops \\
DUT-5(indoline) $)_{1.5}$ (DASA) & $100 \mathrm{mg}$ & $10 \mathrm{~mL}$ & $150 \mathrm{mg}$ & 30 drops \\
DUT-5(indoline) $)_{2.0}$ (DASA) & $100 \mathrm{mg}$ & $10 \mathrm{~mL}$ & $200 \mathrm{mg}$ & 40 drops \\
DUT-5(indoline) $)_{2.5}$ (DASA) & $100 \mathrm{mg}$ & $10 \mathrm{~mL}$ & $250 \mathrm{mg}$ & 50 drops \\
\hline
\end{tabular}




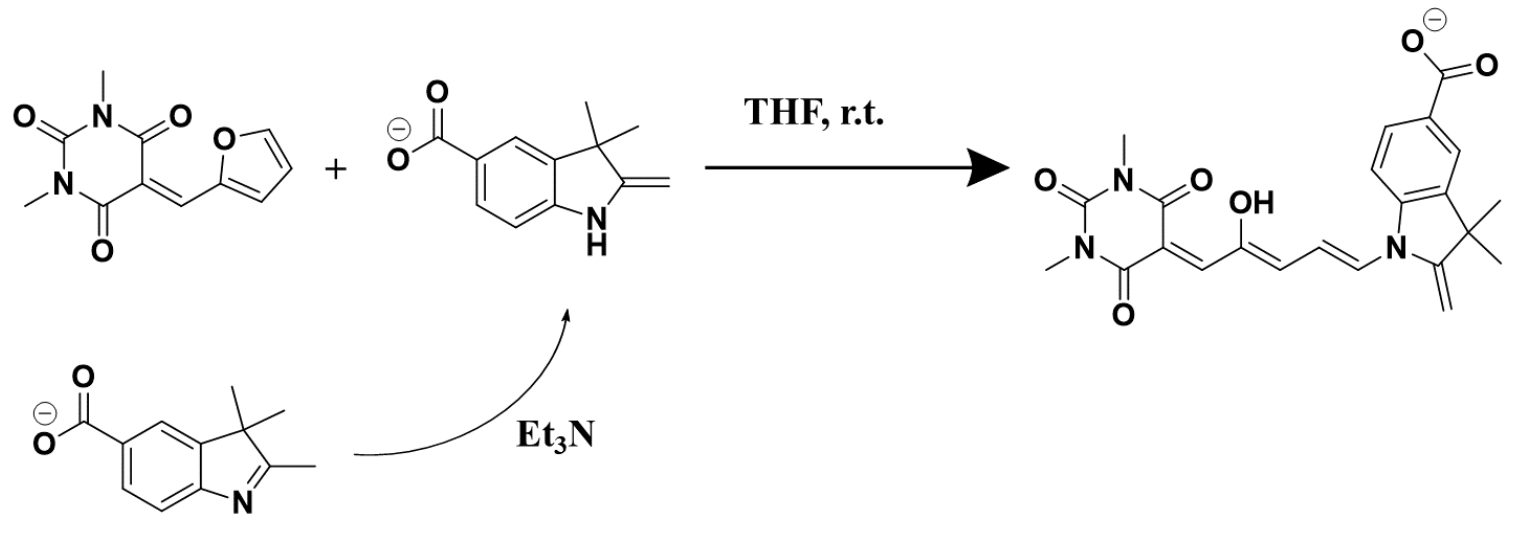

Scheme S1. Proposed reaction scheme for in situ DASA synthesis where $\mathrm{Et}_{3} \mathrm{~N}$ allows $\pi$-bond rearrangement, generating the secondary amine required for photo-switch formation. 
S2. Powder X-Ray Diffraction (PXRD)

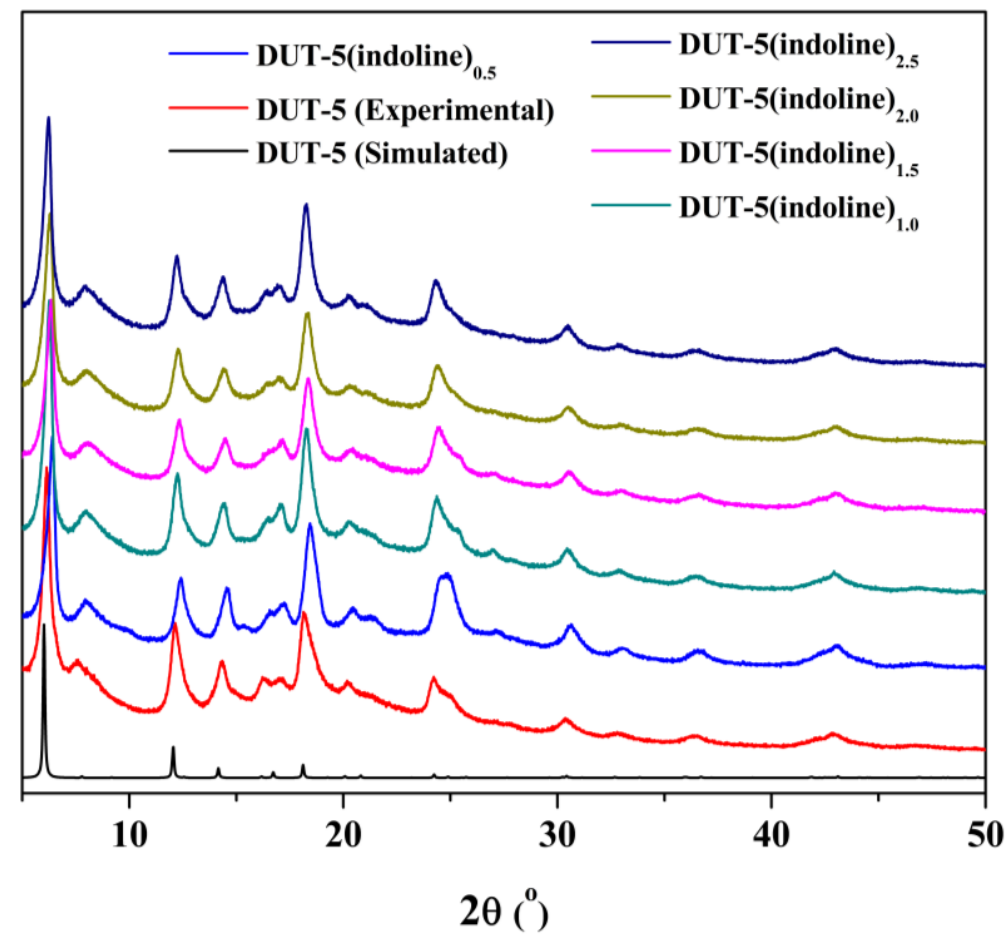

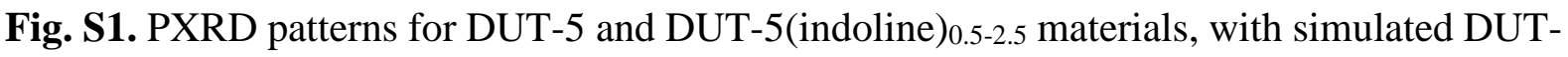
5 pattern provided.

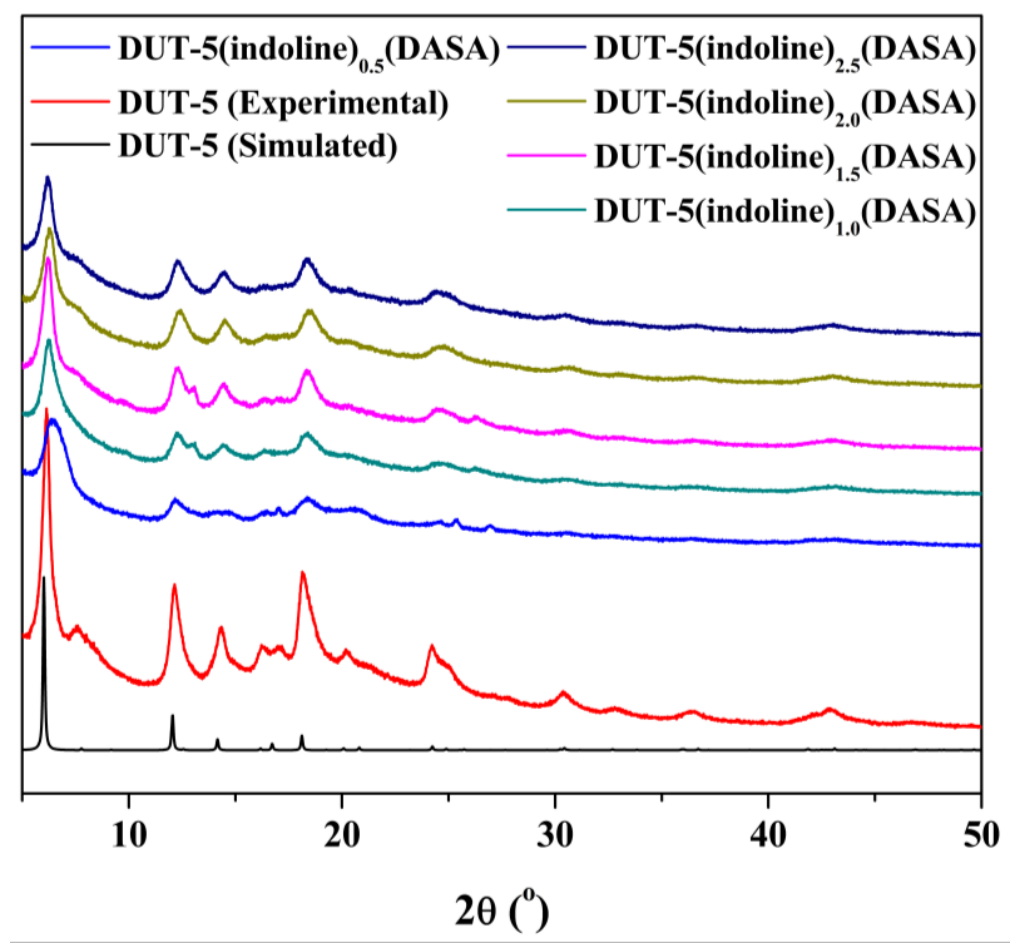

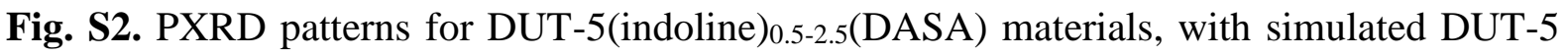
pattern provided. 
Table S2. Cell parameters and refinement details from Le Bail extractions for DUT-5 and DUT-5(indoline $)_{0.5-2.5}$.

\begin{tabular}{|c|c|c|c|c|c|c|}
\hline Parameter & DUT-5 & DUT-5(indoline) $)_{0.5}$ & DUT-5(indoline) $)_{1.0}$ & DUT-5(indoline) $)_{1.5}$ & DUT-5(indoline) $)_{2.0}$ & DUT-5(indoline) $)_{2.5}$ \\
\hline Cell & Orthorhombic & Orthorhombic & Orthorhombic & Orthorhombic & Orthorhombic & Orthorhombic \\
\hline Space Group & Imma & Imma & Imma & Imma & Imma & Imma \\
\hline a $(\AA)$ & $22.71769(11)$ & $22.60962(11)$ & $22.63942(12)$ & $22.60304(18)$ & $22.92373(4)$ & $22.86218(12)$ \\
\hline $\mathrm{b}(\AA)$ & $6.58137(11)$ & $6.58797(11)$ & $6.59121(12)$ & $6.56805(23)$ & $6.57950(10)$ & $6.56144(16)$ \\
\hline$c(\AA)$ & $19.12903(7)$ & $19.28502(7)$ & $19.32977(8)$ & $19.24417(7)$ & $19.0794(23)$ & $18.82737(7)$ \\
\hline$\alpha\left(^{\circ}\right)$ & 90 & 90 & 90 & 90 & 90 & 90 \\
\hline$\beta\left(^{\circ}\right)$ & 90 & 90 & 90 & 90 & 90 & 90 \\
\hline$\gamma\left({ }^{\circ}\right)$ & 90 & 90 & 90 & 90 & 90 & 90 \\
\hline Volume $\left(\AA^{3}\right)$ & $2860.048(7)$ & $2872.532(7)$ & $2884.411(8)$ & $2856.948(9)$ & $2869.923(7)$ & $2824.274(9)$ \\
\hline $\mathrm{R}_{\mathrm{wp}}(\%)$ & 17.388 & 17.928 & 14.899 & 15.730 & 15.044 & 14.868 \\
\hline
\end{tabular}




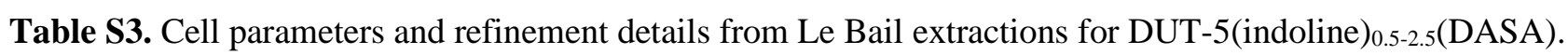

\begin{tabular}{|c|c|c|c|c|c|}
\hline Parameter & DUT-5(indoline) $)_{0.5}($ DASA) & DUT-5(indoline) $)_{1.0}($ DASA) & DUT-5(indoline) $)_{1.5}($ DASA $)$ & DUT-5(indoline) $)_{2.0}($ DASA $)$ & DUT-5(indoline) $)_{2.5}$ (DASA) \\
\hline Cell & Orthorhombic & Orthorhombic & Orthorhombic & Orthorhombic & Orthorhombic \\
\hline Space Group & Imma & Imma & Imma & Imma & Imma \\
\hline $\mathrm{a}(\AA)$ & $22.04267(20)$ & $23.35090(23)$ & $22.91511(4)$ & $23.54490(6)$ & $22.85173(8)$ \\
\hline$b(\AA)$ & $6.80487(22)$ & $6.59764(20)$ & $7.80352(19)$ & $6.58899(18)$ & $8.32485(28)$ \\
\hline c $(\AA)$ & $20.29232(16)$ & $18.44548(12)$ & $17.96707(21)$ & $18.60027(5)$ & $18.50490(5)$ \\
\hline$\alpha\left(^{\circ}\right)$ & 90 & 90 & 90 & 90 & 90 \\
\hline$\beta\left({ }^{\circ}\right)$ & 90 & 90 & 90 & 90 & 90 \\
\hline$\gamma\left({ }^{\circ}\right)$ & 90 & 90 & 90 & 90 & 90 \\
\hline Volume $\left(\AA^{3}\right)$ & $3043.797(12)$ & $2841.726(15)$ & $3123.269(7)$ & $2885.592(9)$ & $3520.322(13)$ \\
\hline $\mathrm{R}_{\mathrm{wp}}(\%)$ & 8.941 & 8.949 & 6.447 & 10.773 & 9.750 \\
\hline
\end{tabular}


a)
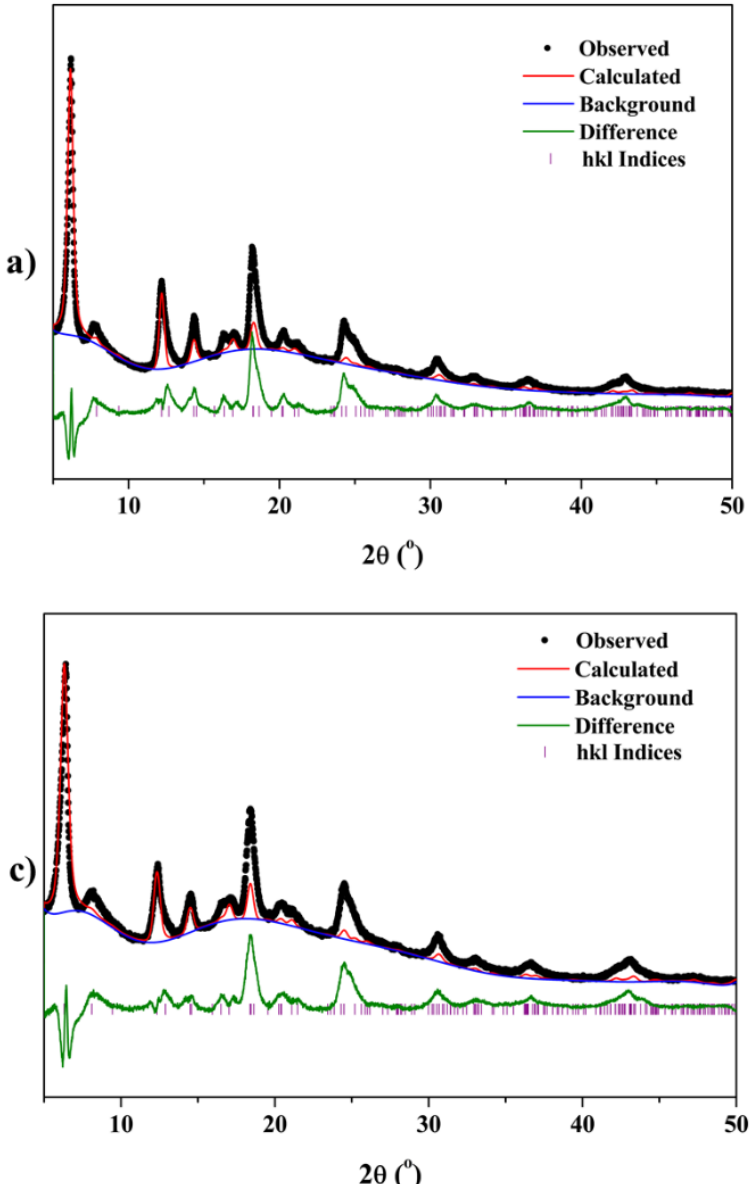

e)

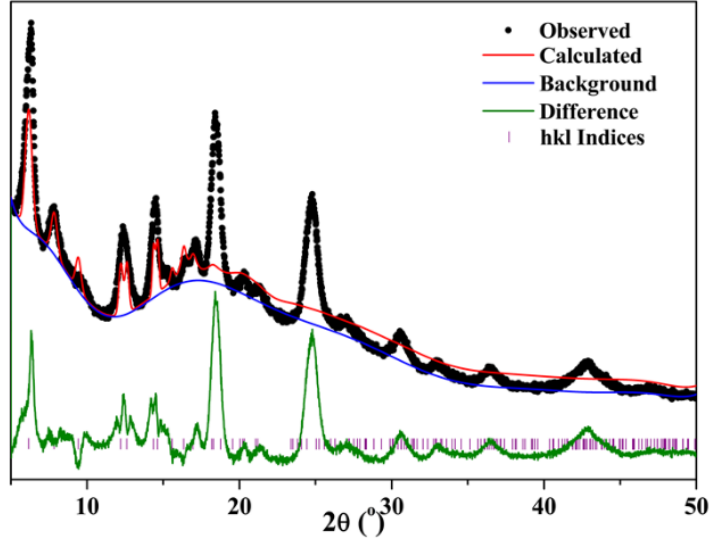

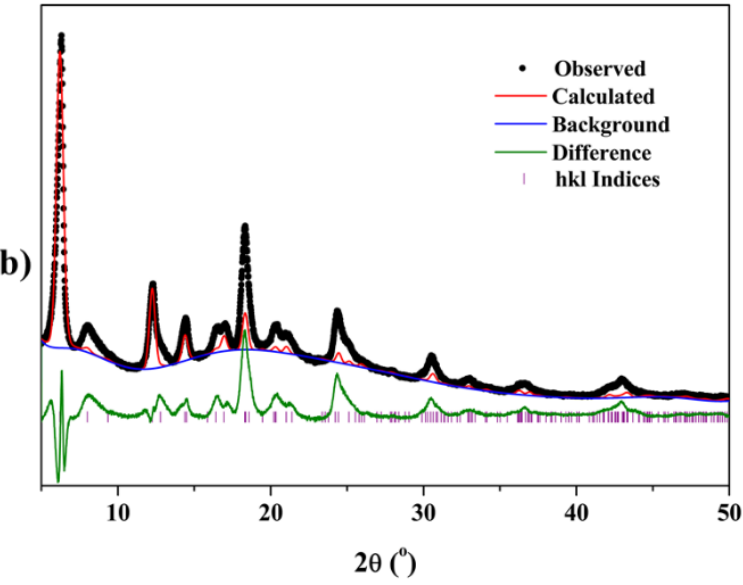
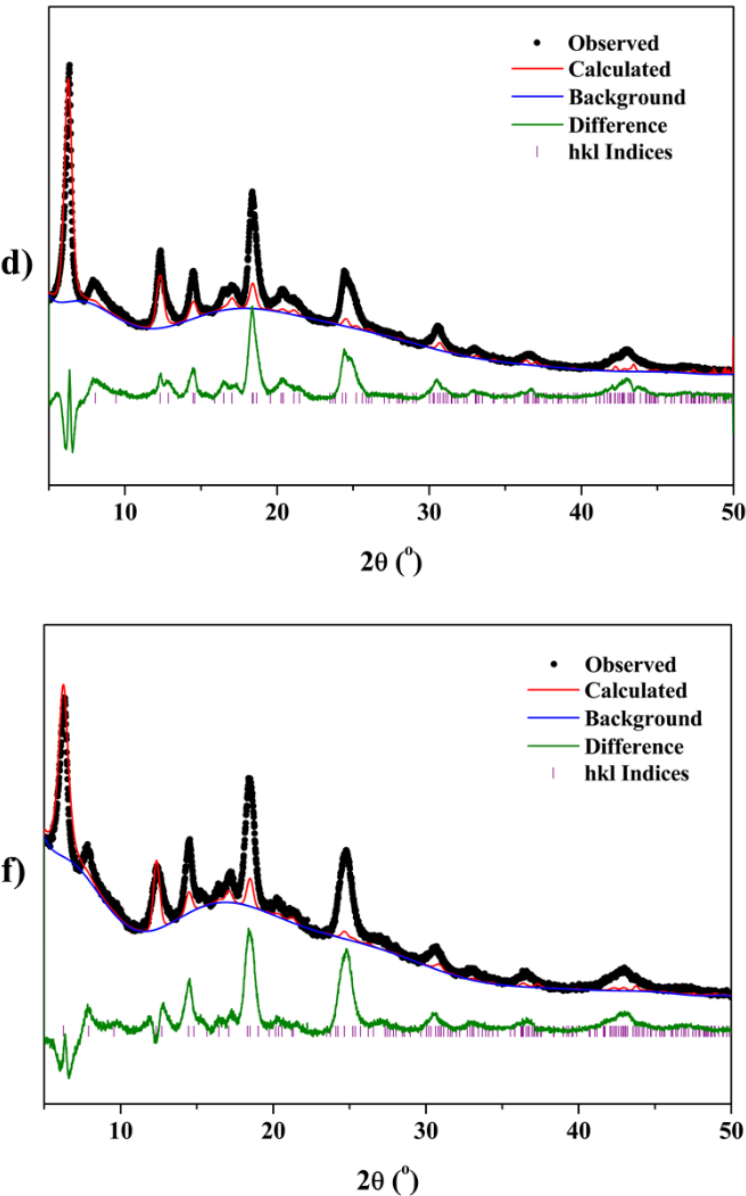

Fig. S3. Le Bail extractions for DUT-5 (a) and DUT-5(indoline) $0.5-2.5$ (b-f). 
a)
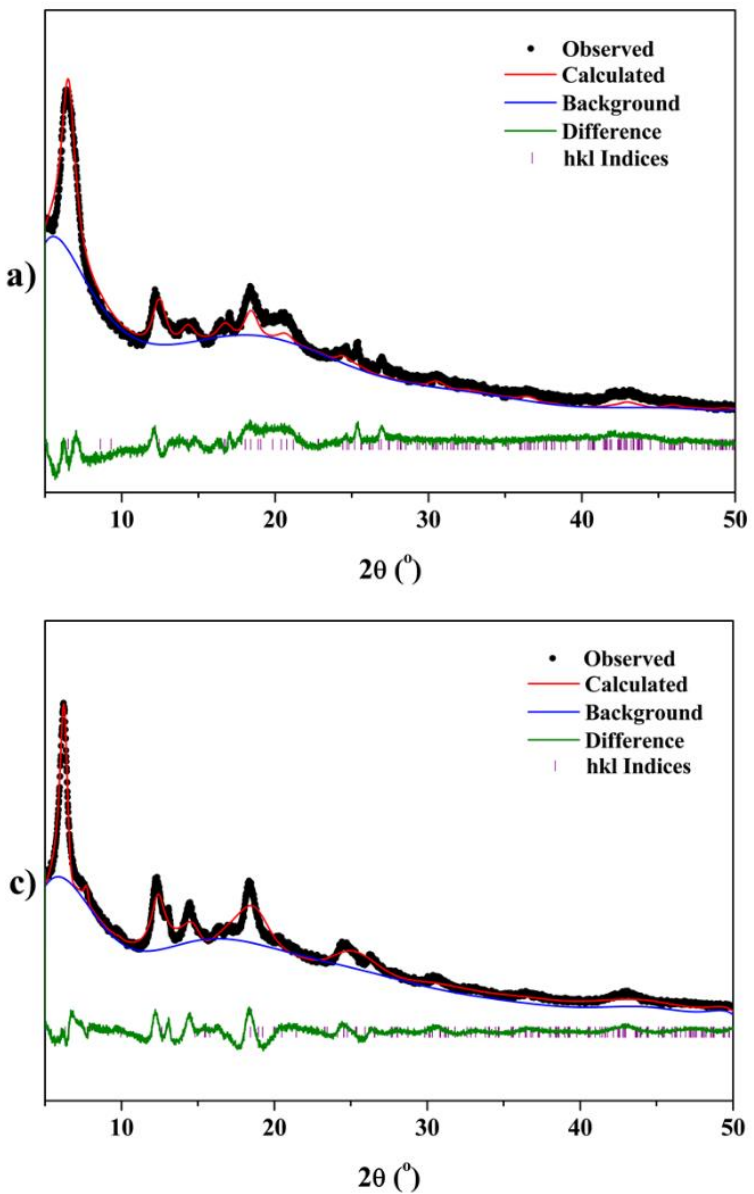
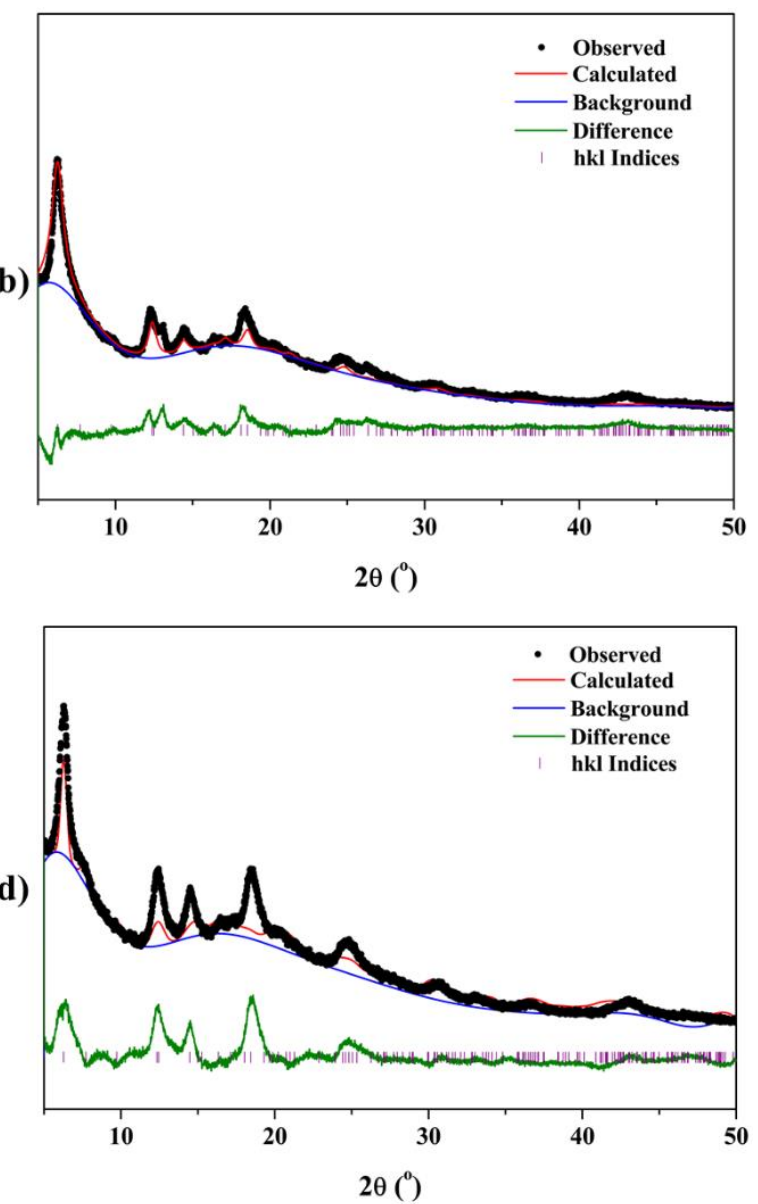

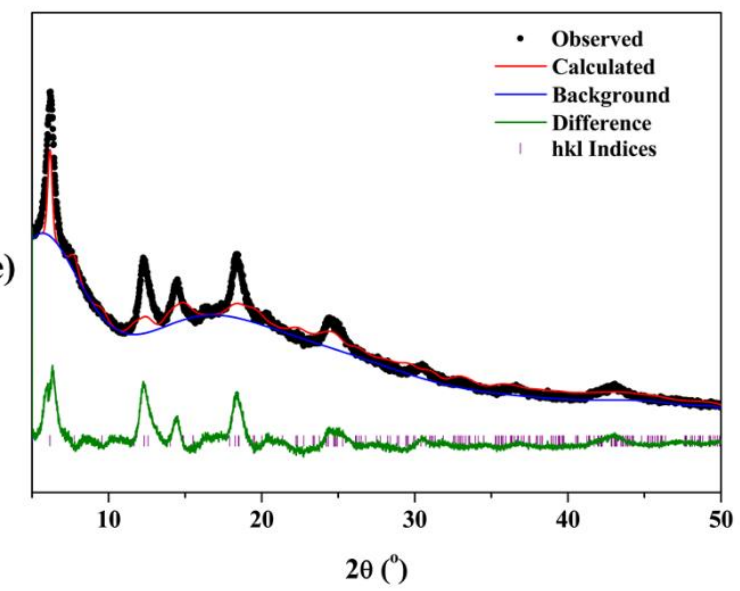

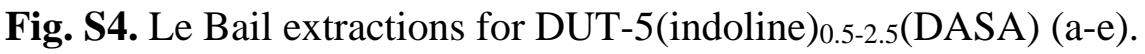




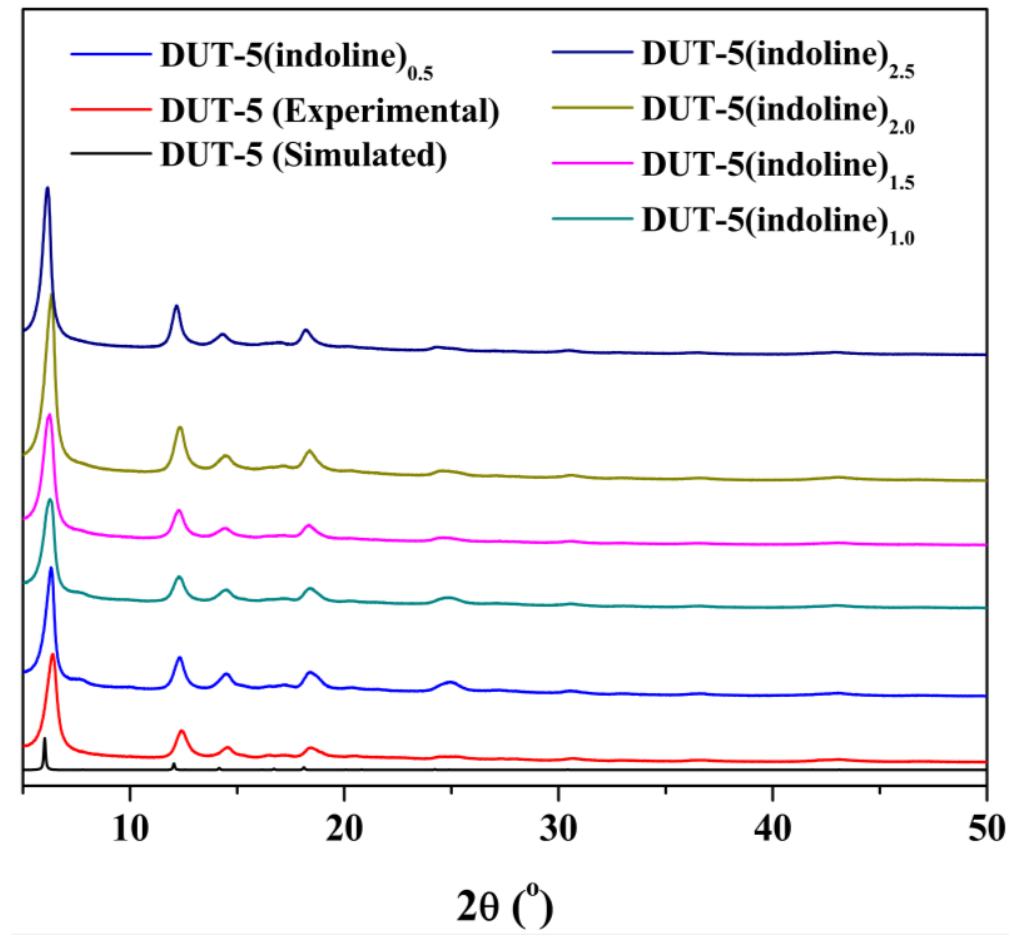

Fig. S5. PXRD patterns for DUT-5 and DUT-5(indoline) $0.5-2.5$ materials following gas sorption analyses, with simulated DUT-5 pattern provided.

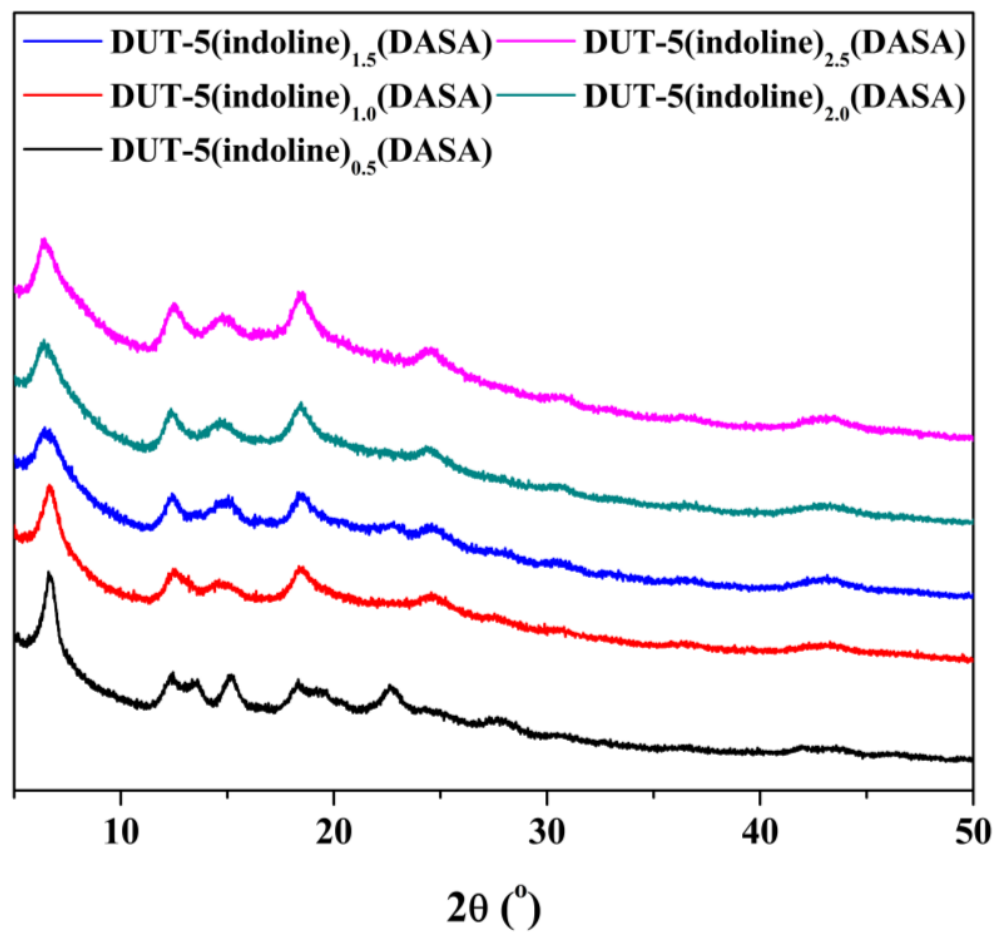

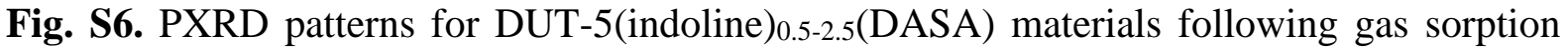
analyses. 


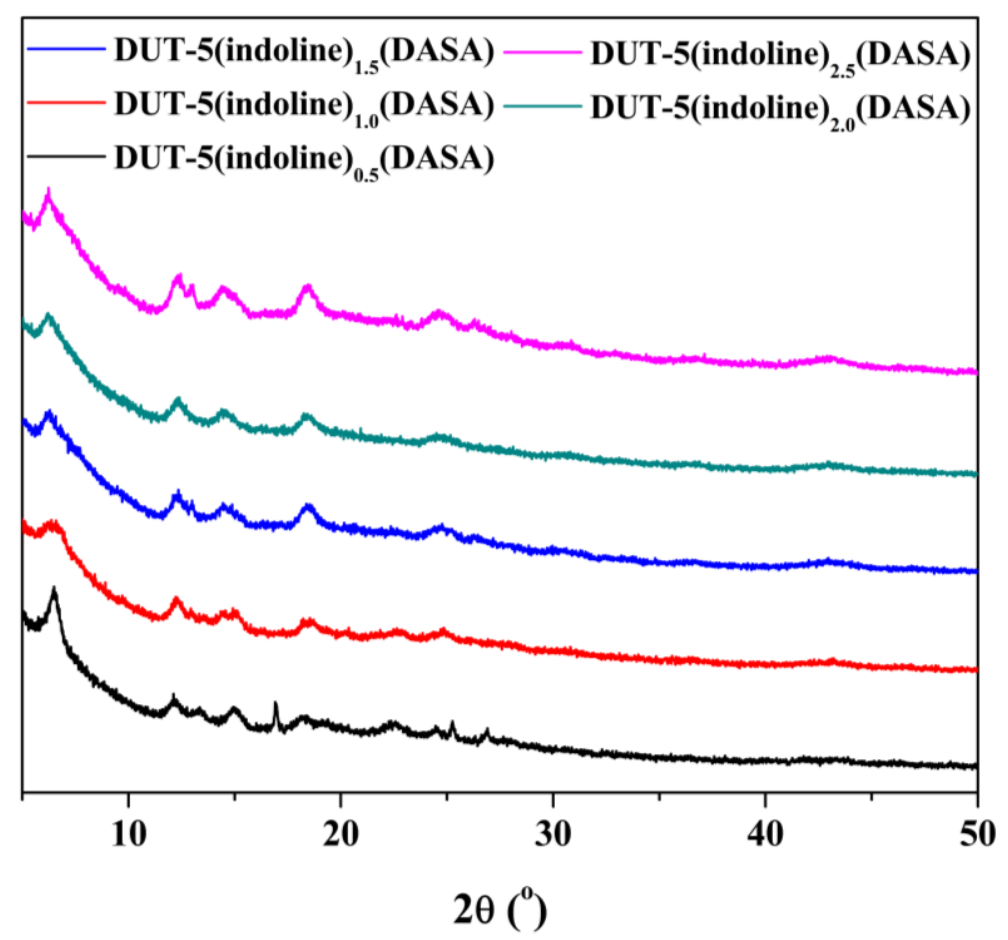

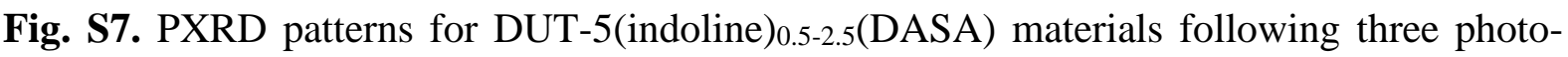
switching cycles.

PXRD patterns for DUT-5 and the derivative series were consistent with that detailed in the literature (Fig. S1 and S2). ${ }^{6}$ This assessment is made despite the reduction in crystallinity in the DUT-5(indoline) $)_{0.5-2.5}$ (DASA) series based on other literature examples indicating less than optimum crystallinity. ${ }^{7}$ In addition to the lack of ideal crystallinity being intrinsic to the DUT5 architecture, the displacement of bpdc $^{2-}$ linker was expected to be responsible for partial deterioration as observed for other Al(III) series. ${ }^{8}$ Furthermore, cell parameters (Tables S2 and S3) calculated from Pawley extractions were still consistent with original description provided by Senkovska et al. ${ }^{6}$ As can be observed in Fig. S3 and S4, Pawley fits from which cell parameters were derived are comparatively poor. This is attributable to the difficulties in obtaining an ideal crystalline product for the DUT-5 framework which is further compounded by the disorder initially imparted as part of defect generation.

PXRD was used for confirming architectural stability of the MOFs across a range of analyses. This includes gas sorption analyses where it was determined that the architectures were maintained throughout surface area measurements (Fig. S5 and S6). Finally, stability of the defective DUT-5(indoline) $0.5-2.5$ (DASA) required confirmation following three switching cycles (Fig. S7). 
S3. ${ }^{1}$ H Nuclear Magnetic Resonance (NMR) Spectroscopy
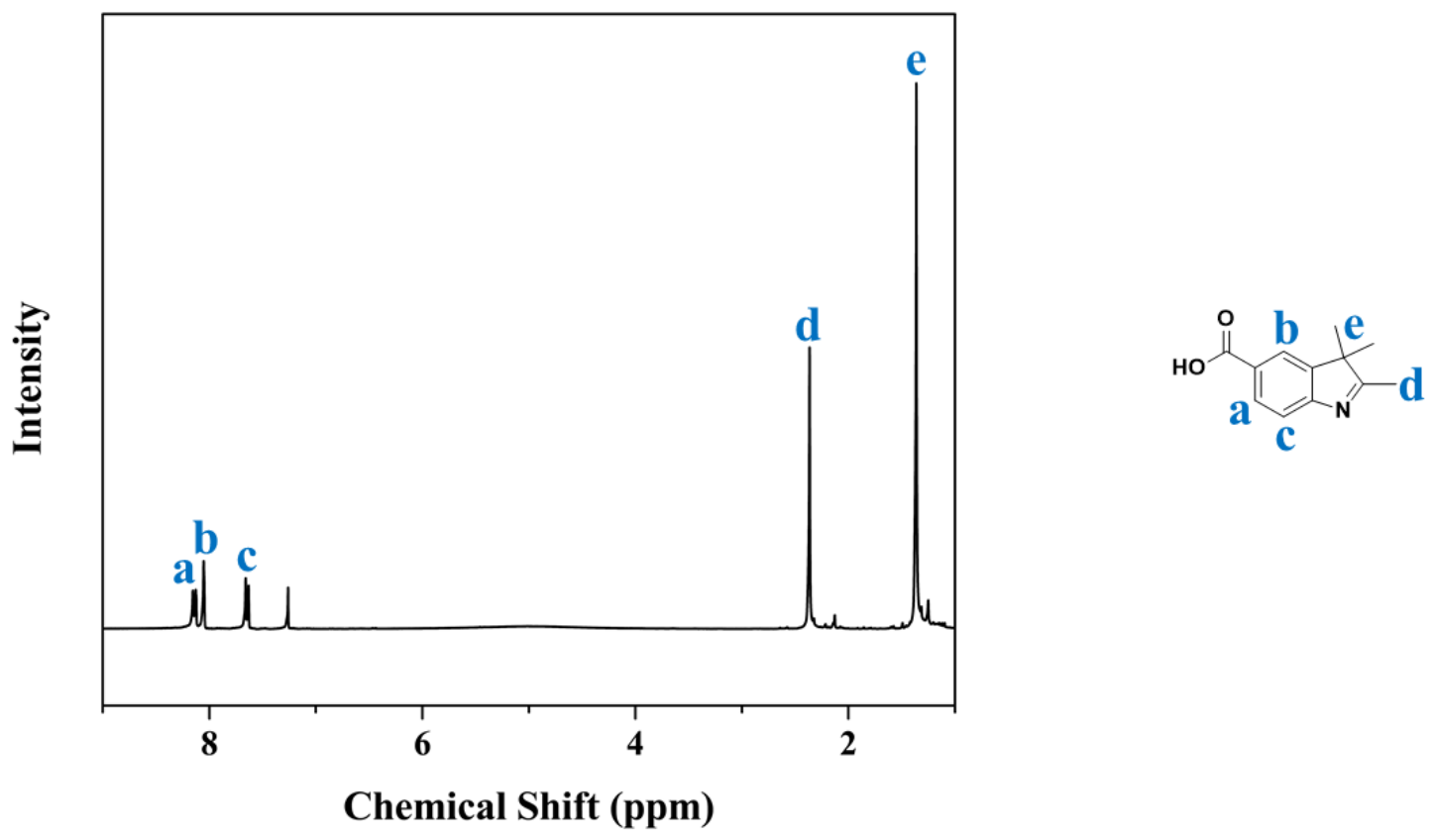

Figure S8. $300 \mathrm{MHz}{ }^{1} \mathrm{H}$ NMR spectrum for indoline $\left(\mathrm{CDCl}_{3}\right)$.
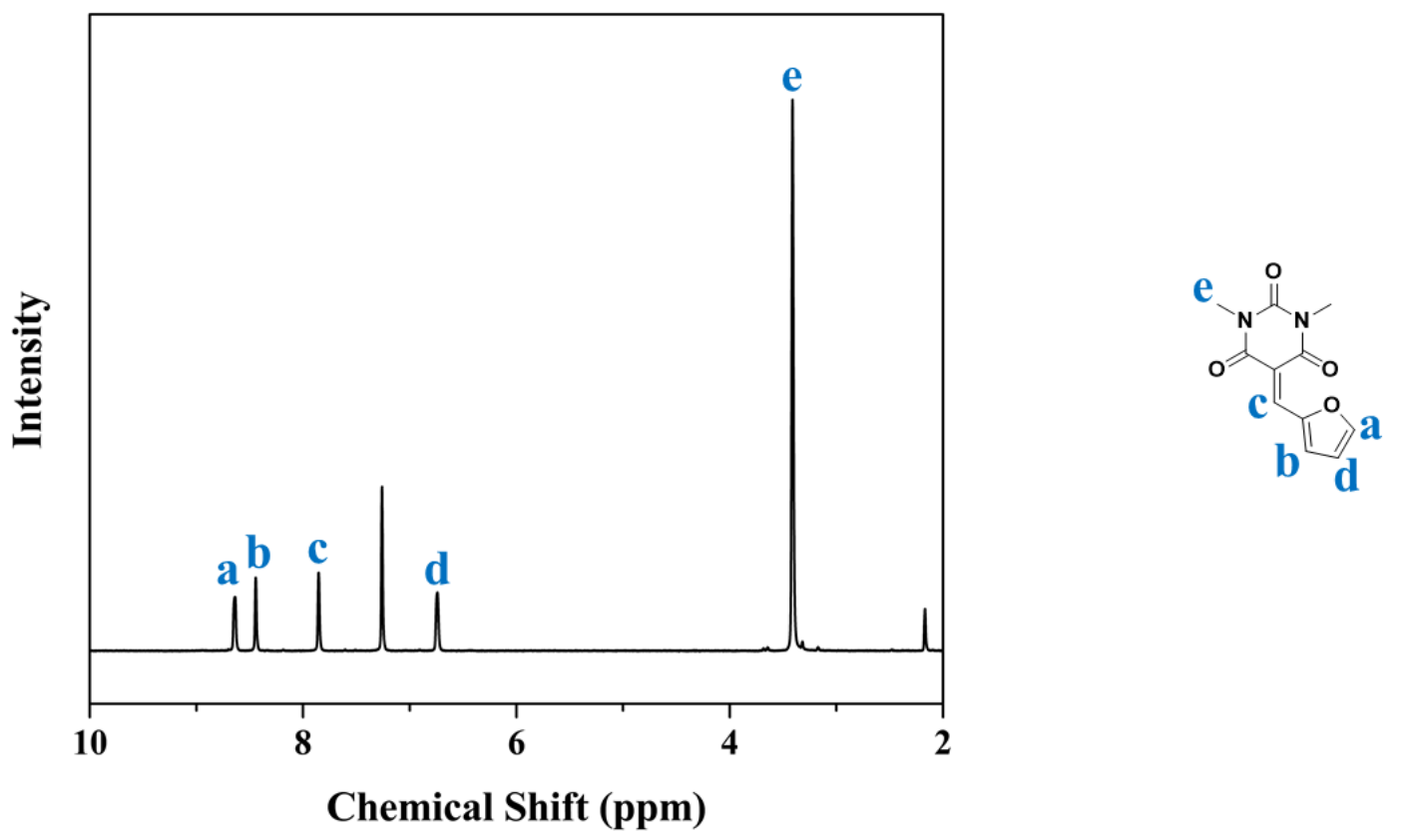

Fig. S9. $300 \mathrm{MHz}{ }^{1} \mathrm{H}$ NMR spectrum for $\mathrm{Me}_{2} \mathrm{DP}\left(\mathrm{CDCl}_{3}\right)$. 

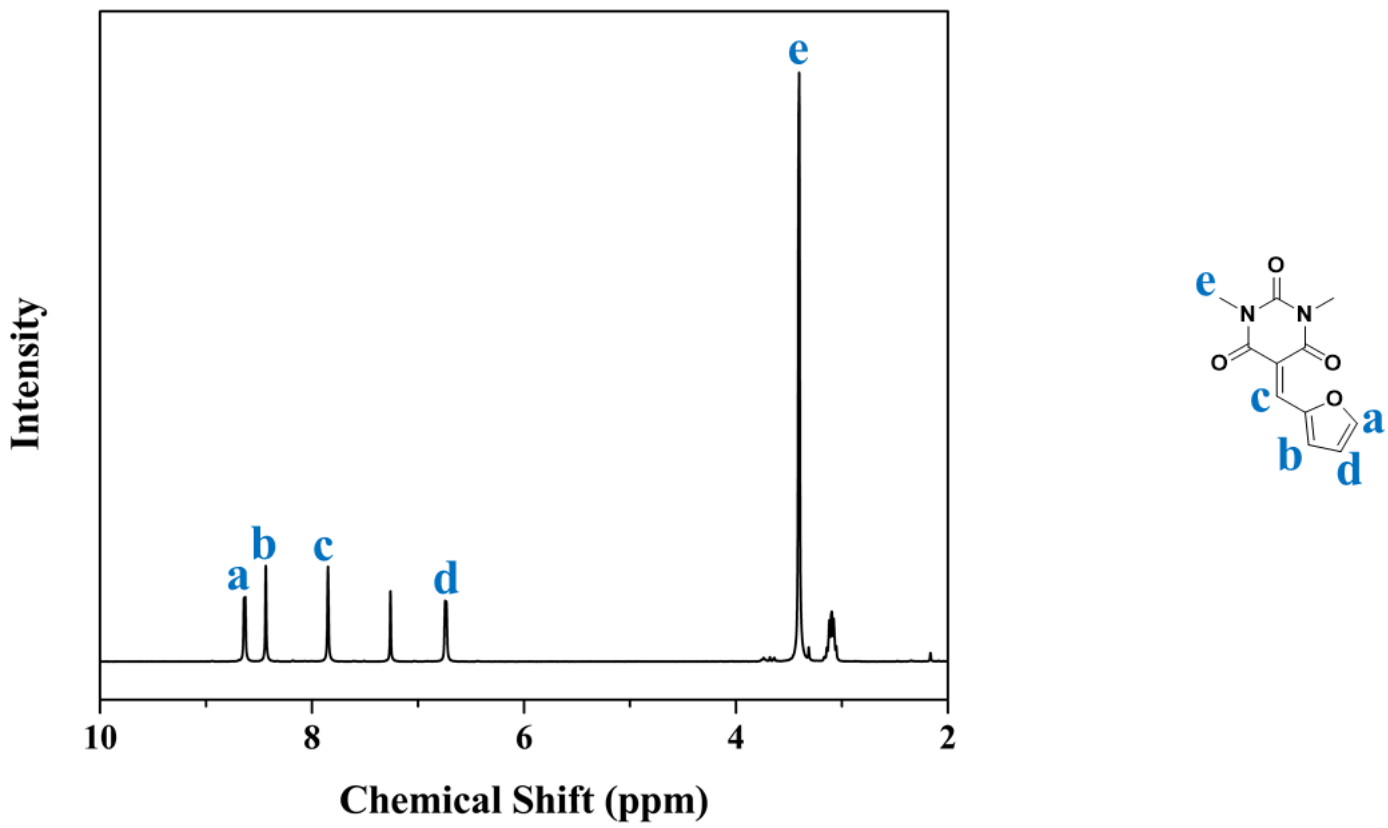

Fig. S10. $300 \mathrm{MHz}{ }^{1} \mathrm{H}$ NMR spectrum for DASA ${ }_{I N D}\left(\mathrm{CDCl}_{3}\right)$.
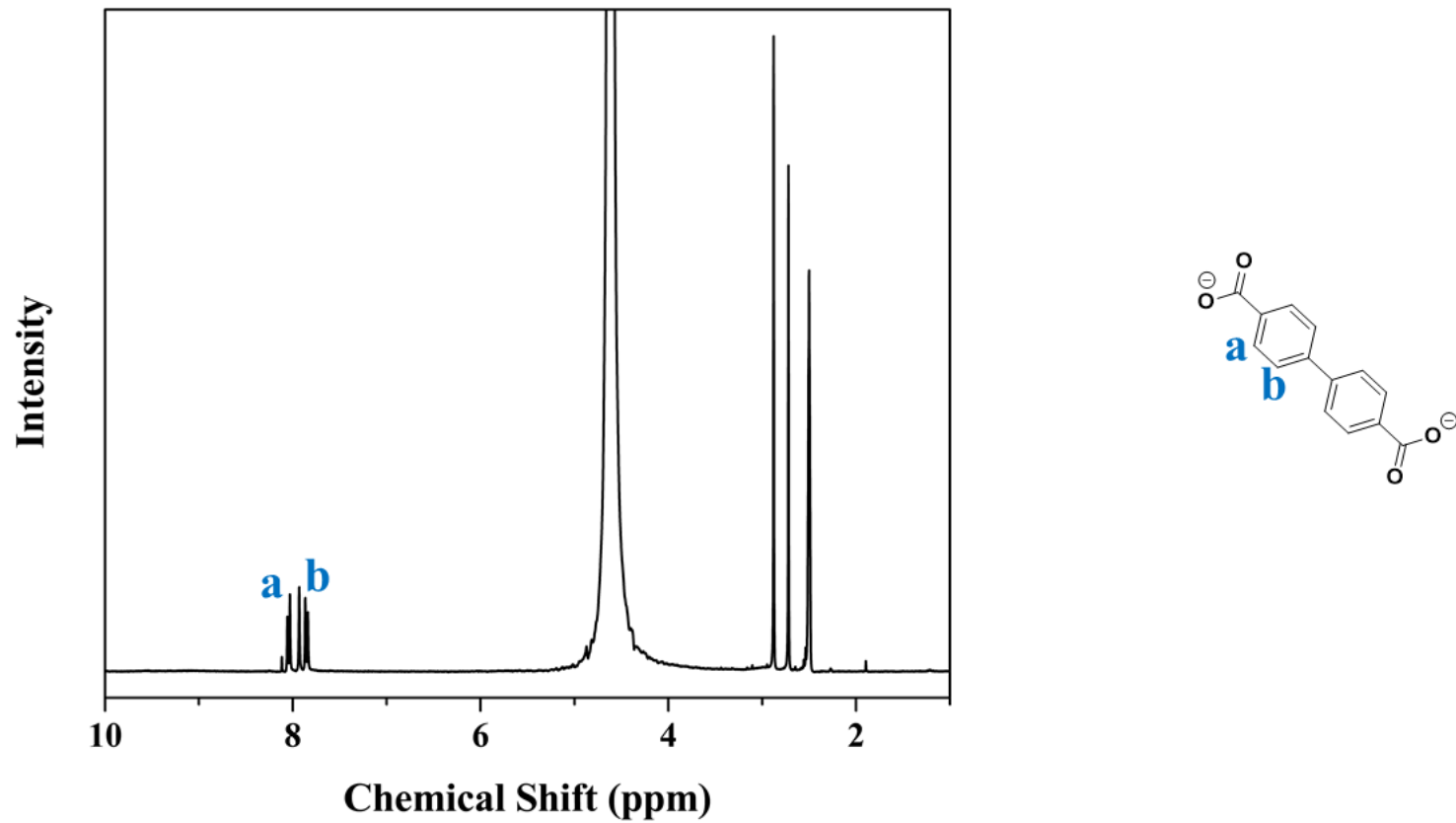

Fig. S11. $300 \mathrm{MHz}{ }^{1} \mathrm{H}$ NMR spectrum for digested DUT-5 (DMSO- $d_{6} / \mathrm{TFA}$ ). 

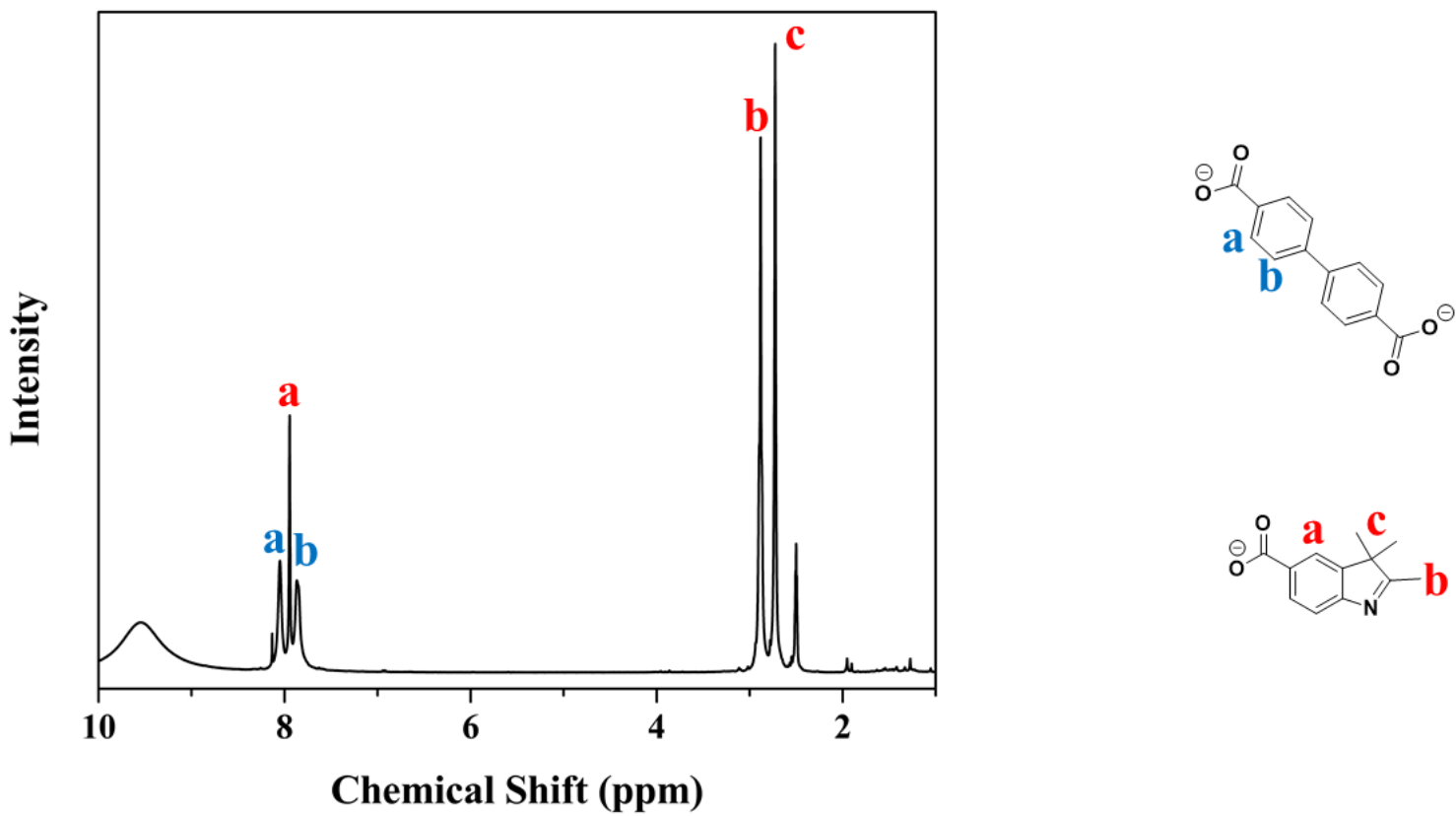

Fig. S12. $300 \mathrm{MHz}{ }^{1} \mathrm{H}$ NMR spectrum for digested DUT-5(indoline) $)_{0.5}$ (DMSO-d $/$ TFA).
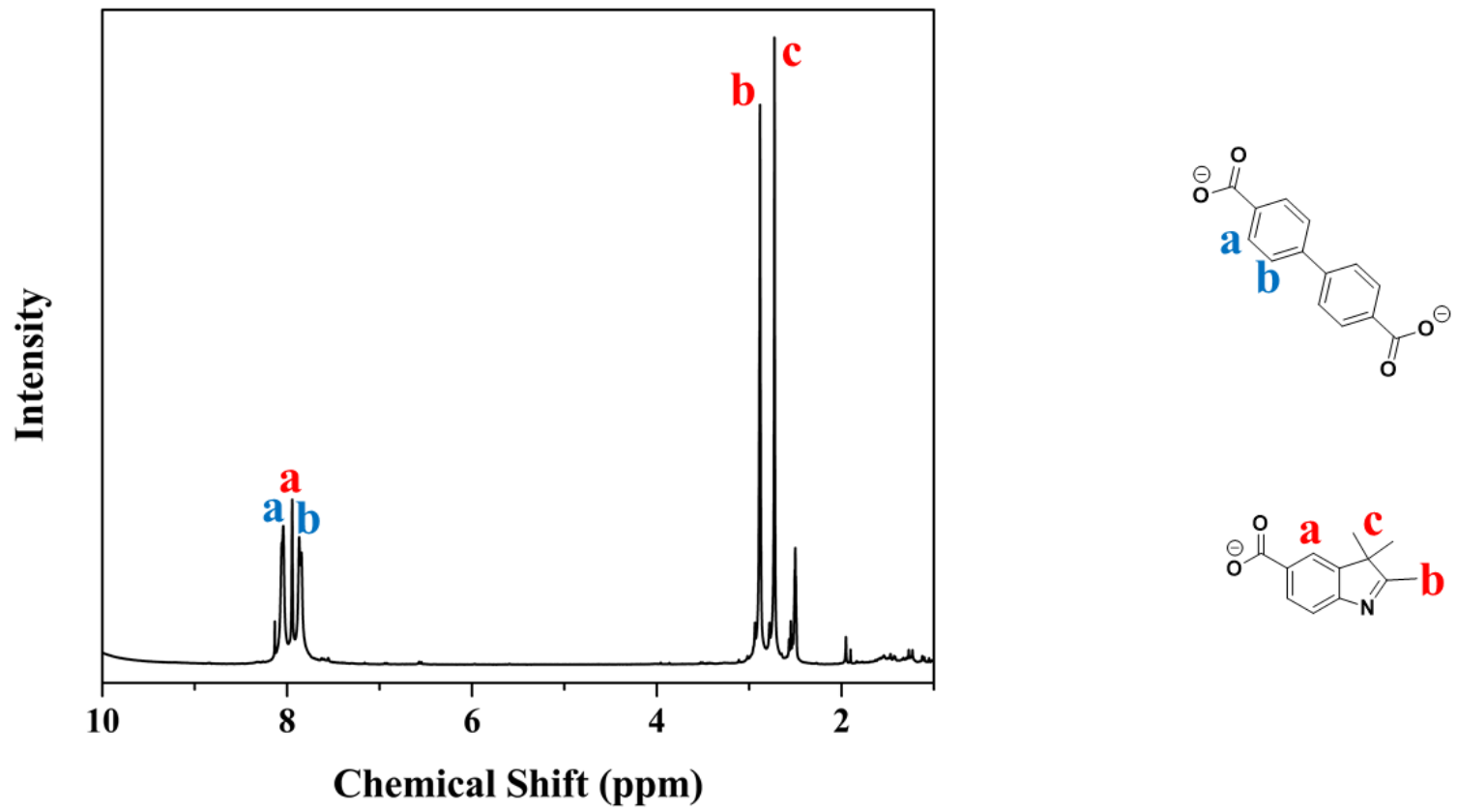

Fig. S13. $300 \mathrm{MHz}{ }^{1} \mathrm{H}$ NMR spectrum for digested DUT-5(indoline) $)_{1.0}$ (DMSO- $d_{6} / \mathrm{TFA}$ ). 


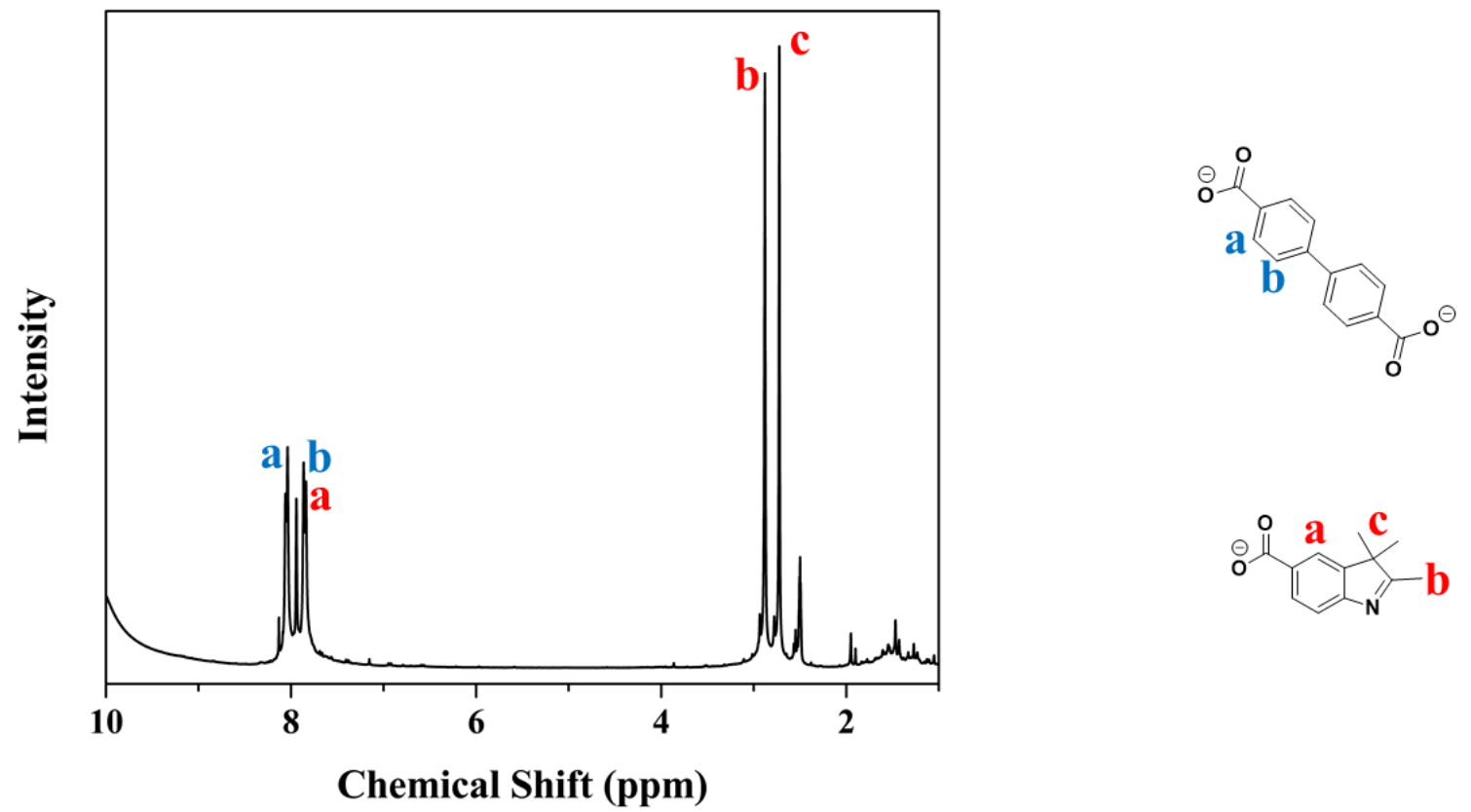

Fig. S14. $300 \mathrm{MHz}{ }^{1} \mathrm{H}$ NMR spectrum for digested DUT-5(indoline) $)_{1.5}$ (DMSO- $d_{6} / \mathrm{TFA}$ ).
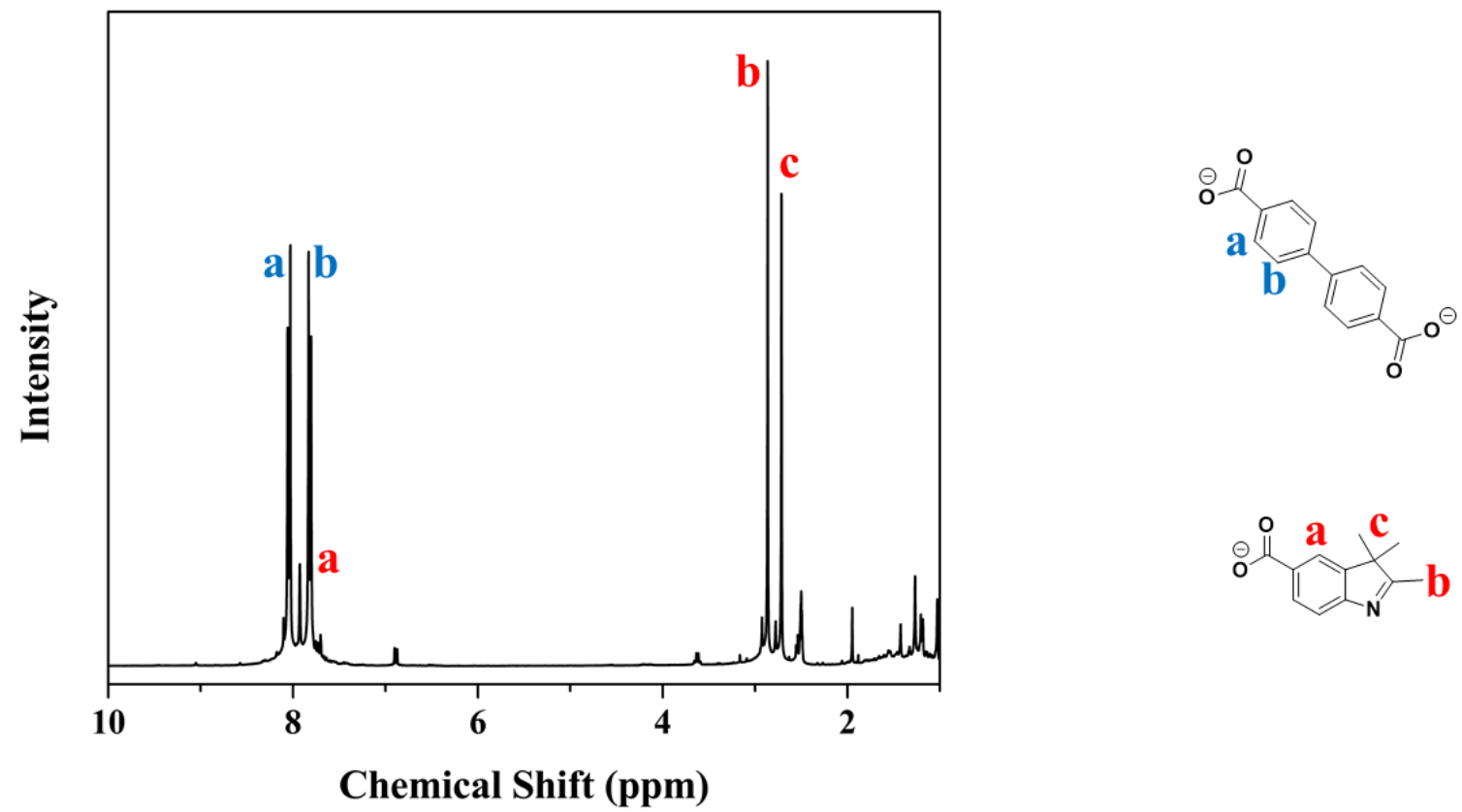

Fig. S15. $300 \mathrm{MHz}{ }^{1} \mathrm{H}$ NMR spectrum for digested DUT-5(indoline) $)_{2.0}$ (DMSO- $d_{6} / \mathrm{TFA}$ ). 


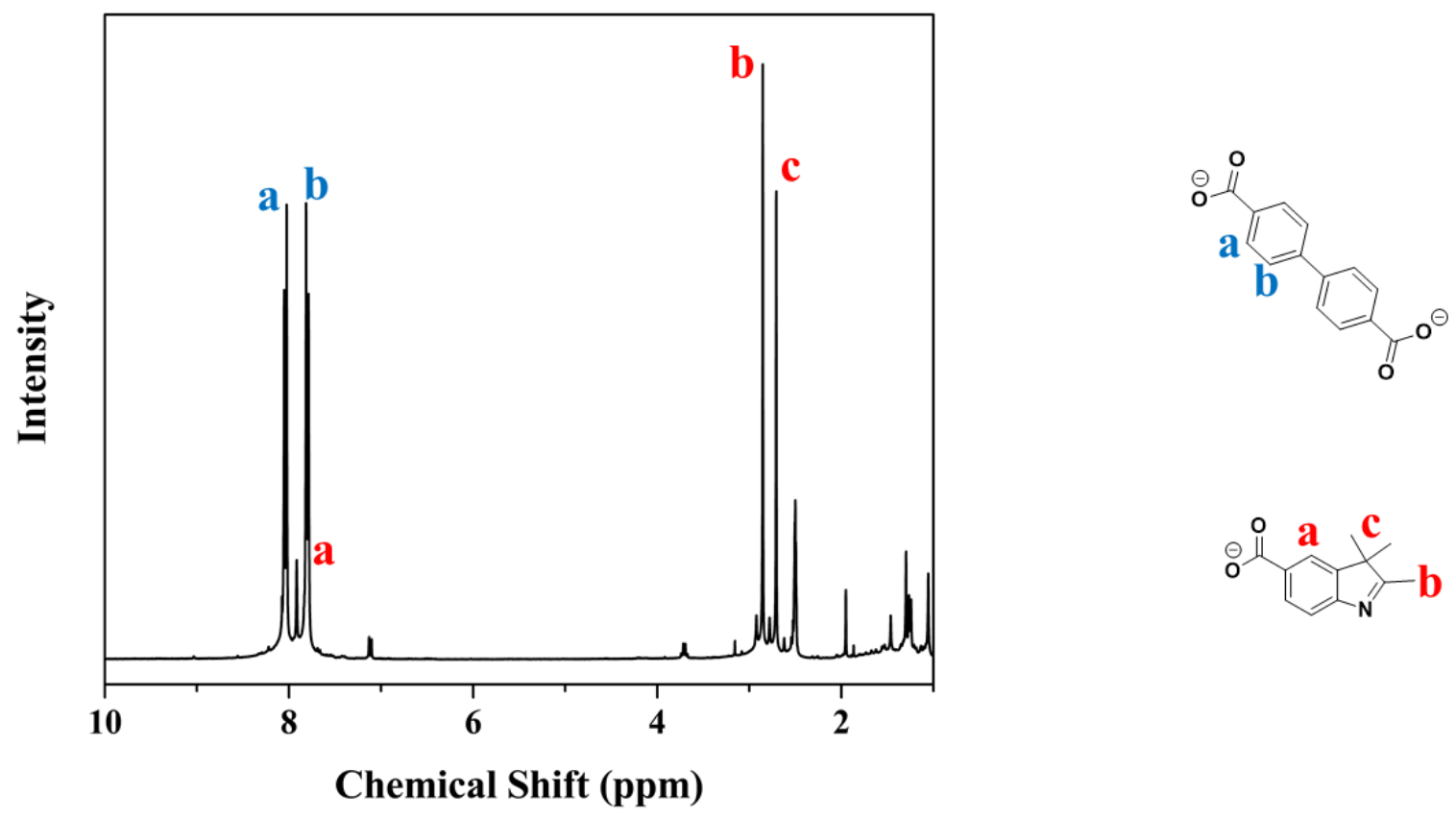

Fig. S16. $300 \mathrm{MHz}{ }^{1} \mathrm{H}$ NMR spectrum for digested DUT-5(indoline) 2.5 (DMSO- $d_{6} / \mathrm{TFA}$ ).
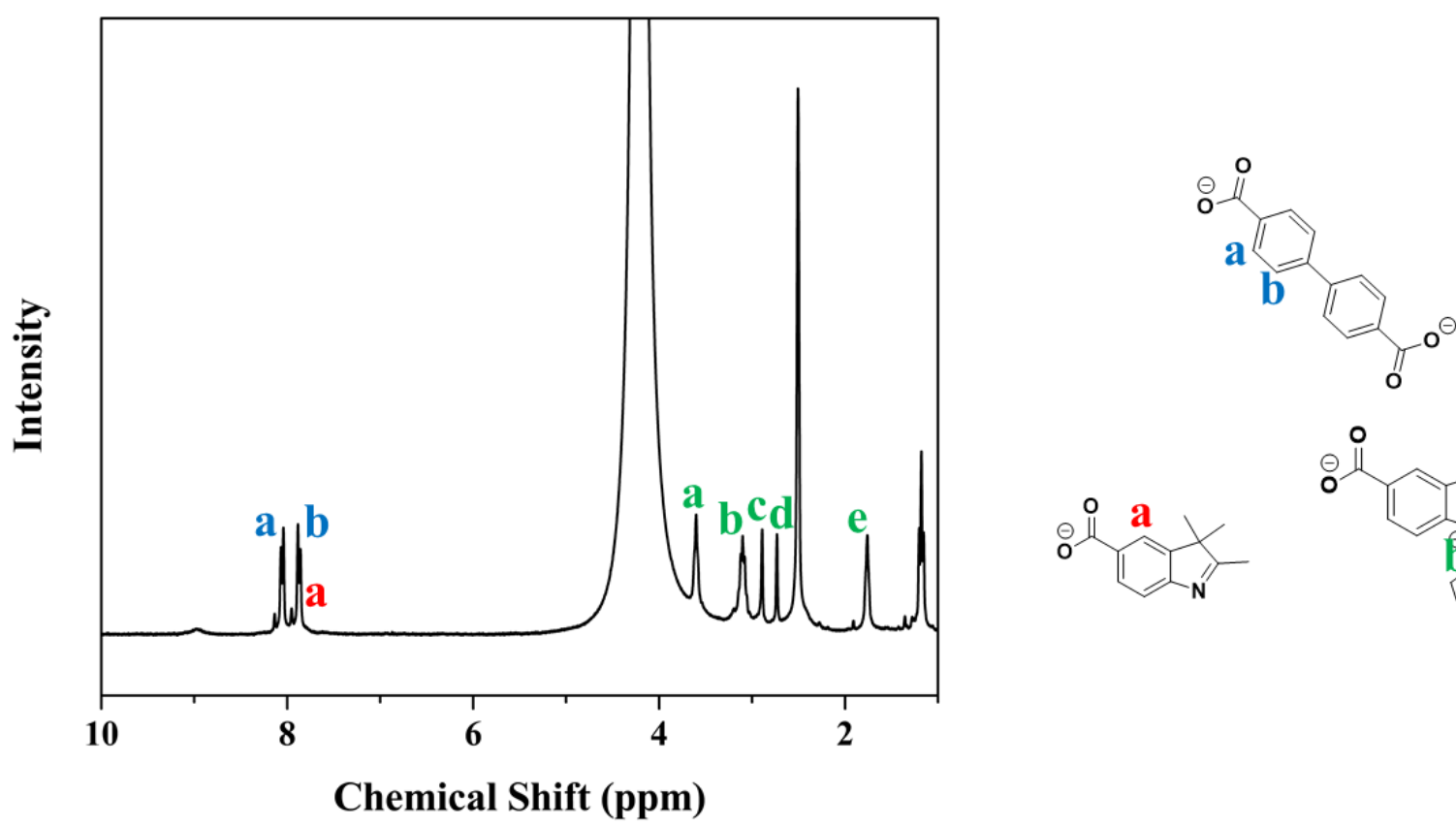

Fig. S17. $300 \mathrm{MHz}$ solid-state ${ }^{1} \mathrm{H}$ NMR spectrum for digested DUT-5(indoline) 0.5 (DASA) (DMSO- $d_{6} /$ TFA). 


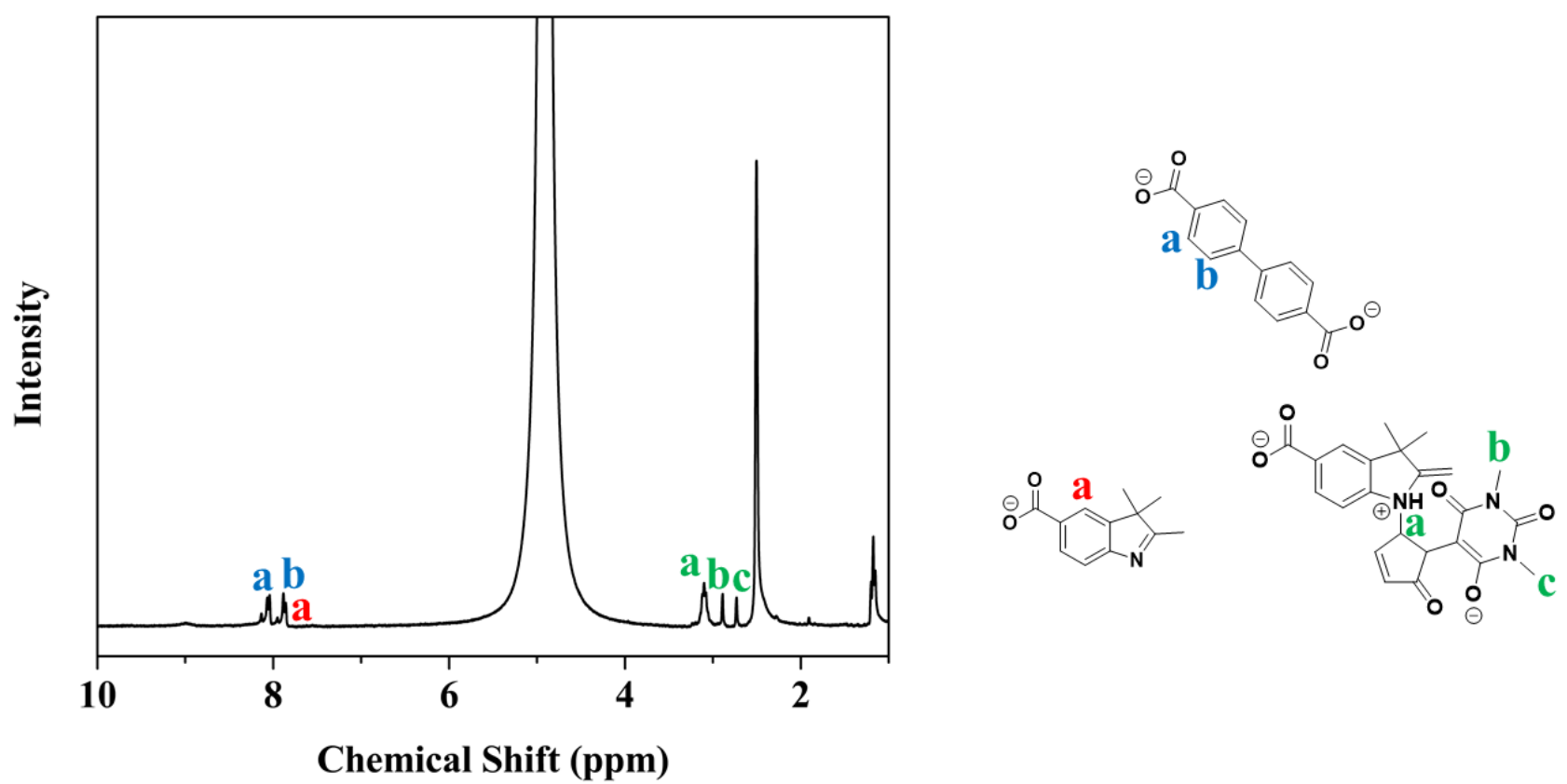

Fig. S18. $300 \mathrm{MHz}$ solid-state ${ }^{1} \mathrm{H}$ NMR spectrum for digested DUT-5(indoline) 1.0 $_{\text {(DASA) }}$ (DMSO- $d_{6} / \mathrm{TFA}$ ).
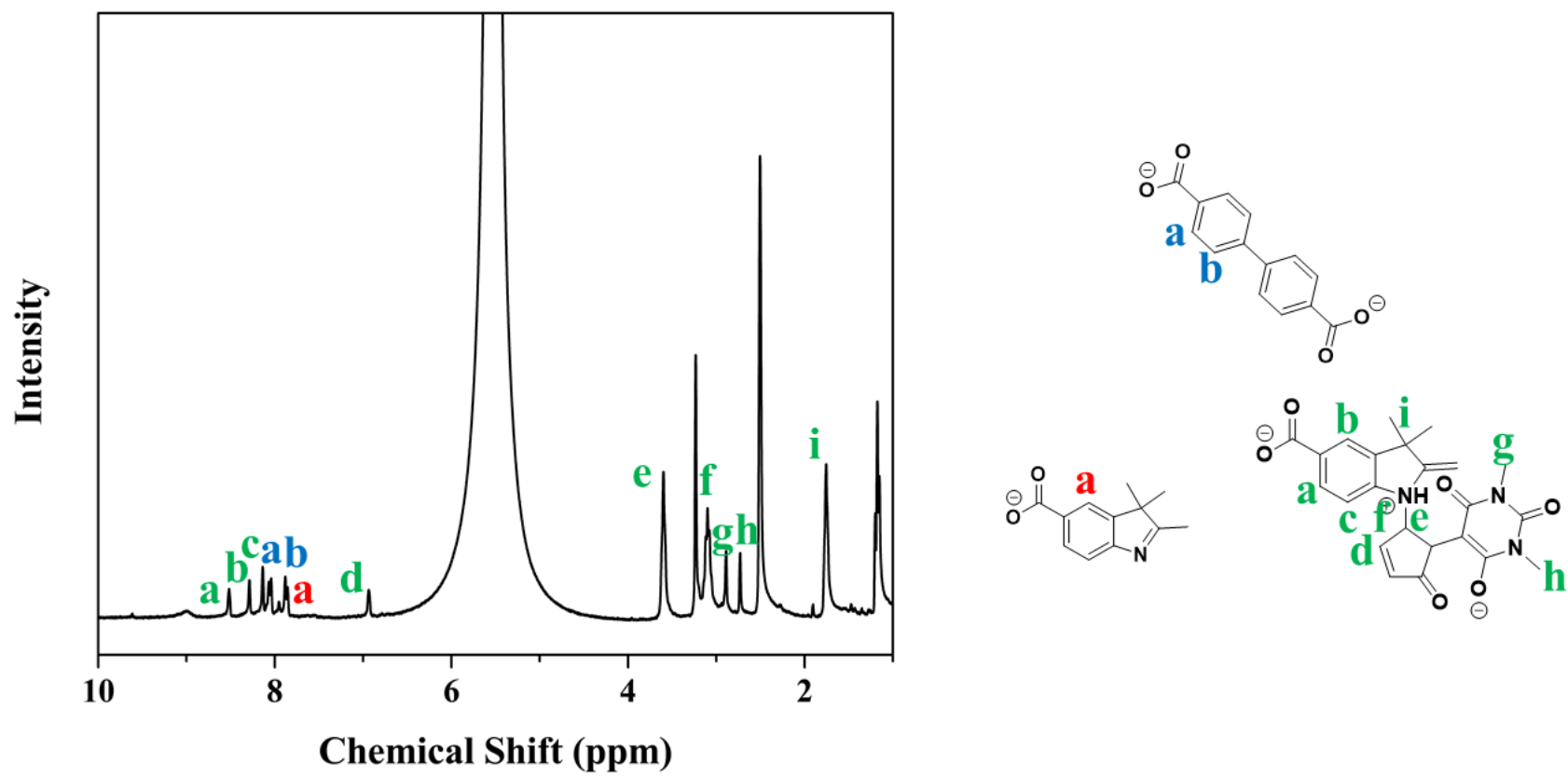

Fig. S19. $300 \mathrm{MHz}$ solid-state ${ }^{1} \mathrm{H}$ NMR spectrum for digested DUT-5(indoline) 1.5 (DASA) $^{2}$ (DMSO- $\left.d_{6} / \mathrm{TFA}\right)$. 

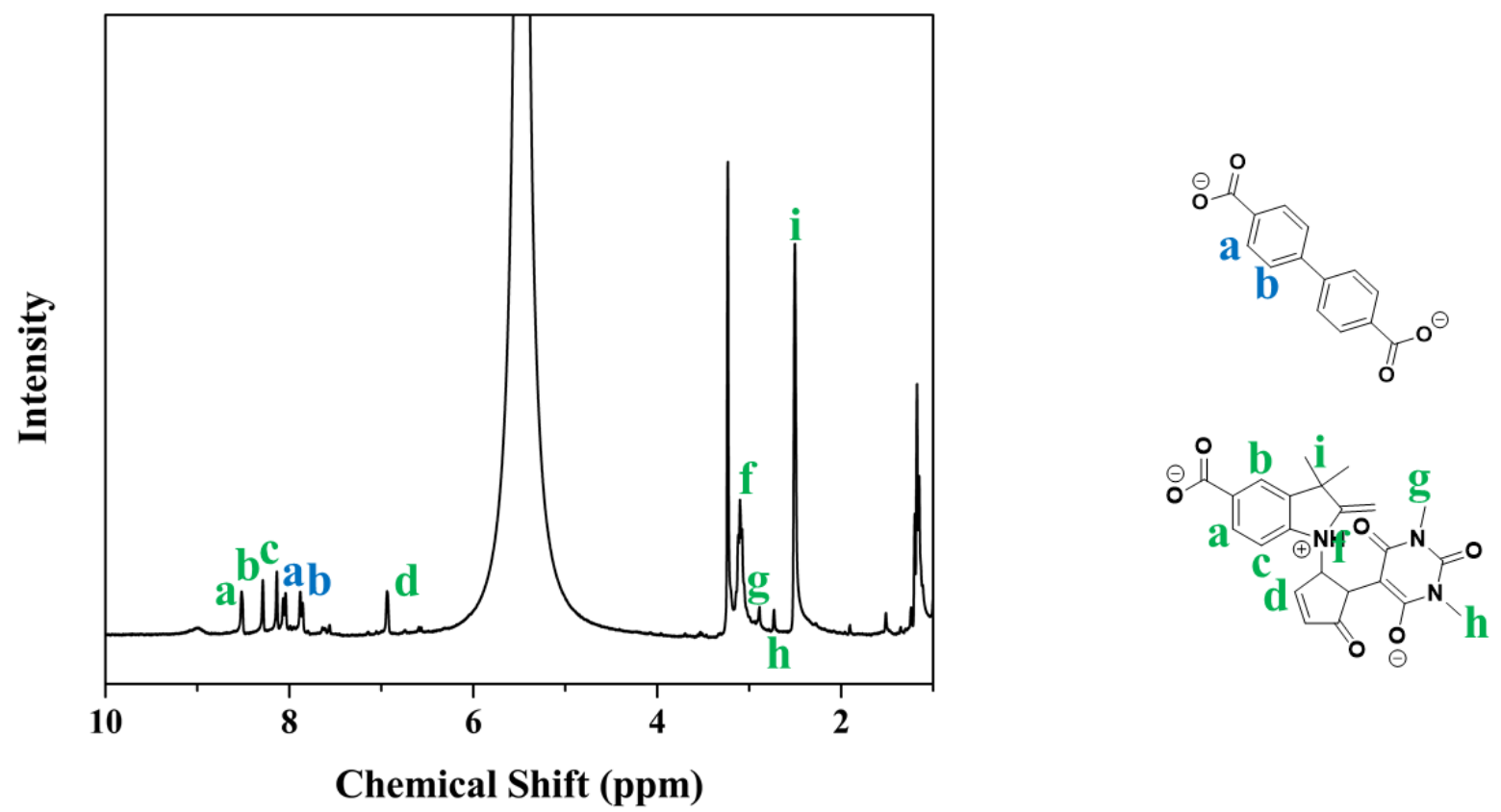

Fig. S20. $300 \mathrm{MHz}$ solid-state ${ }^{1} \mathrm{H}$ NMR spectrum for digested DUT-5(indoline) 2.0 (DASA) (DMSO- $d_{6} / \mathrm{TFA}$ ).
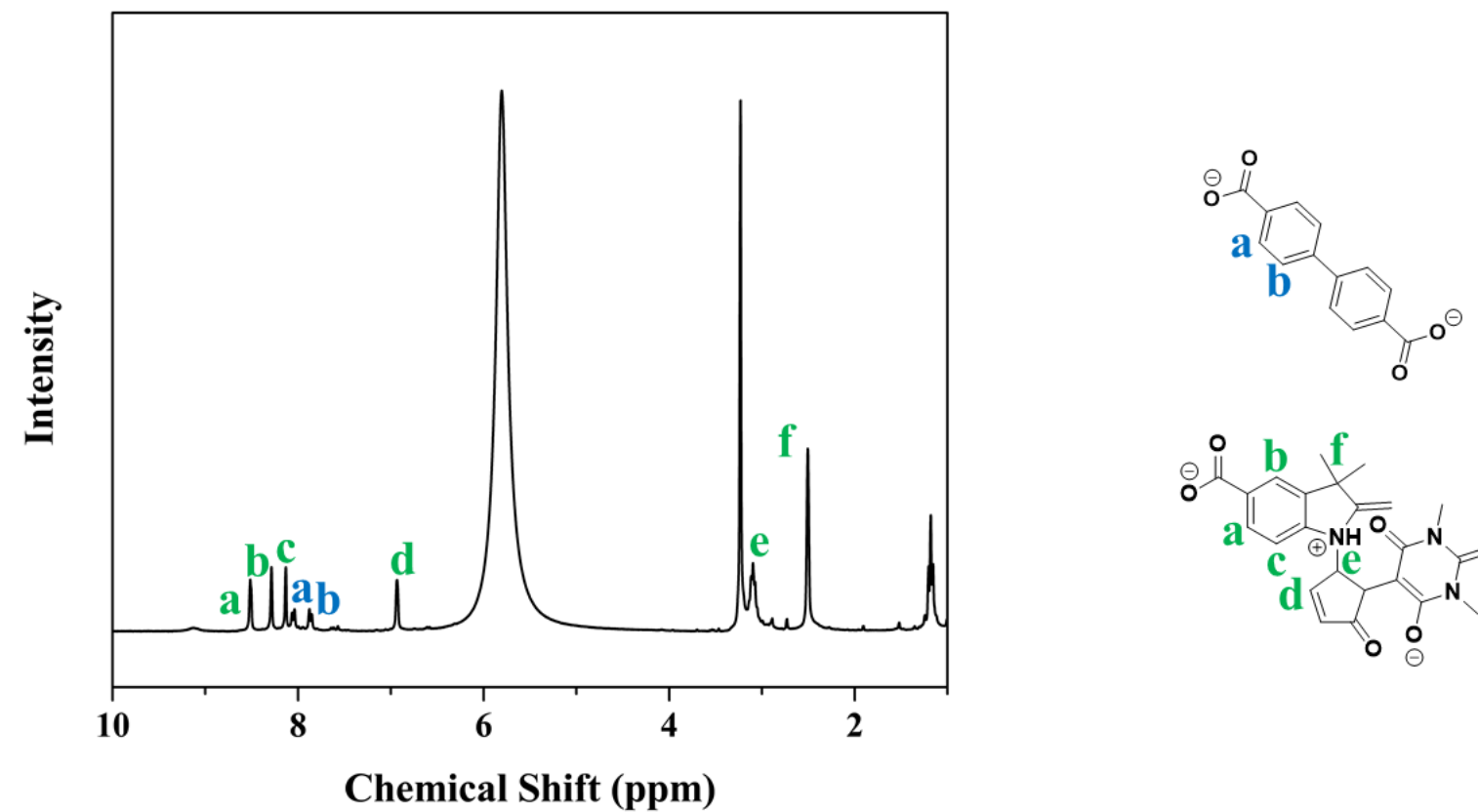

Fig. S21. $300 \mathrm{MHz}$ solid-state ${ }^{1} \mathrm{H}$ NMR spectrum for digested DUT-5(indoline)2.5(DASA) (DMSO- $\left.d_{6} / \mathrm{TFA}\right)$. 


\section{S4. Electrospray Ionisation Mass Spectrometry (ESI-MS)}
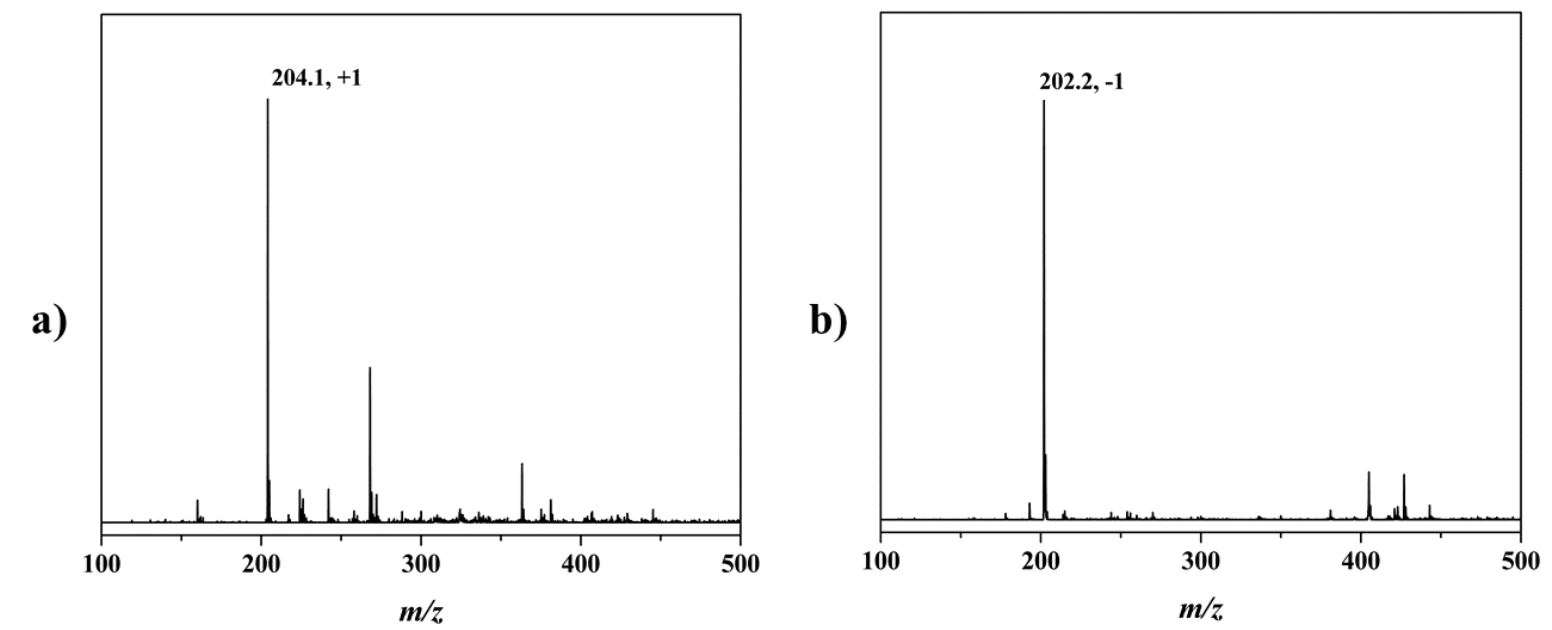

Fig. S22. Positive (a) and negative (b) ion ESI-MS spectra for indoline in $\mathrm{MeOH}$; where $\mathrm{m} / \mathrm{z}=$ $204.1[\mathrm{M}+1]^{+}$and $202.2[\mathrm{M}-1]^{-}$.

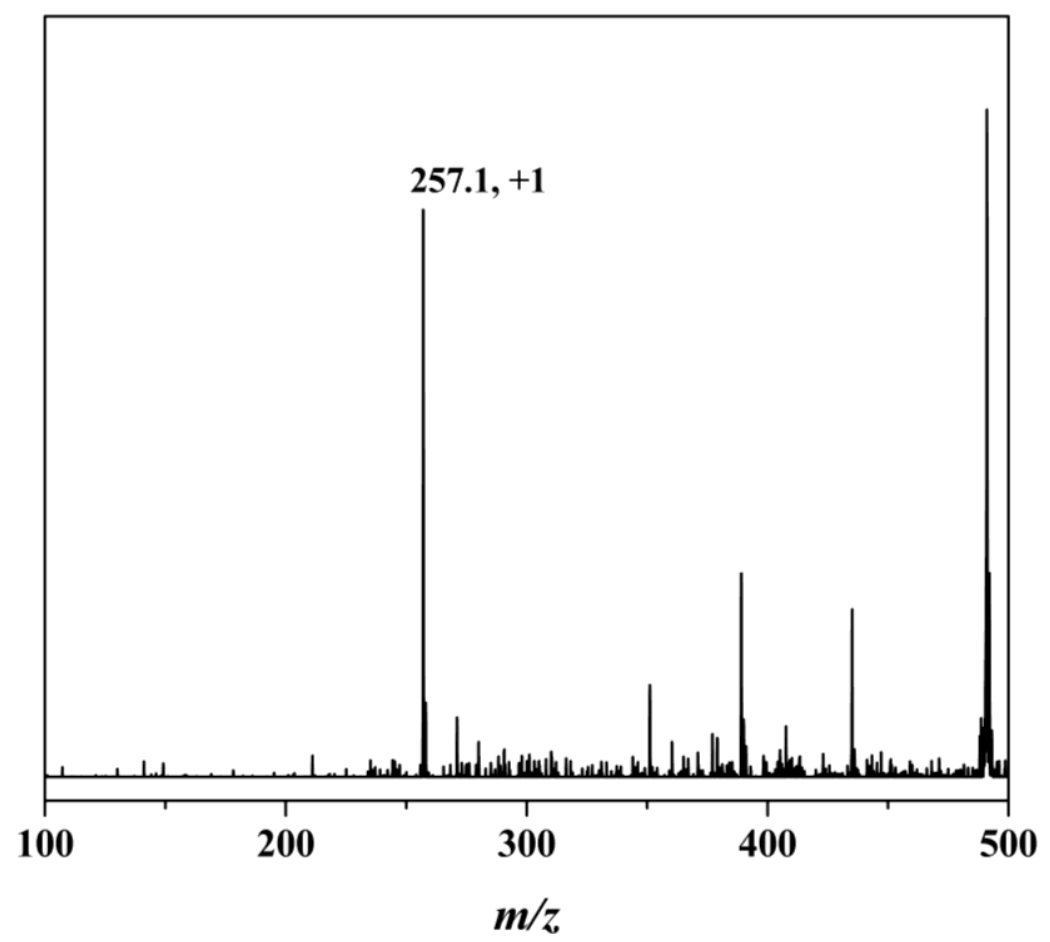

Fig. S23. Positive ion ESI-MS spectrum for $\mathrm{Me}_{2} \mathrm{DP}$ in $\mathrm{MeOH}$; where $m / z=257.1\left[\mathrm{M}+\mathrm{Na}^{+}\right]^{+}$. 


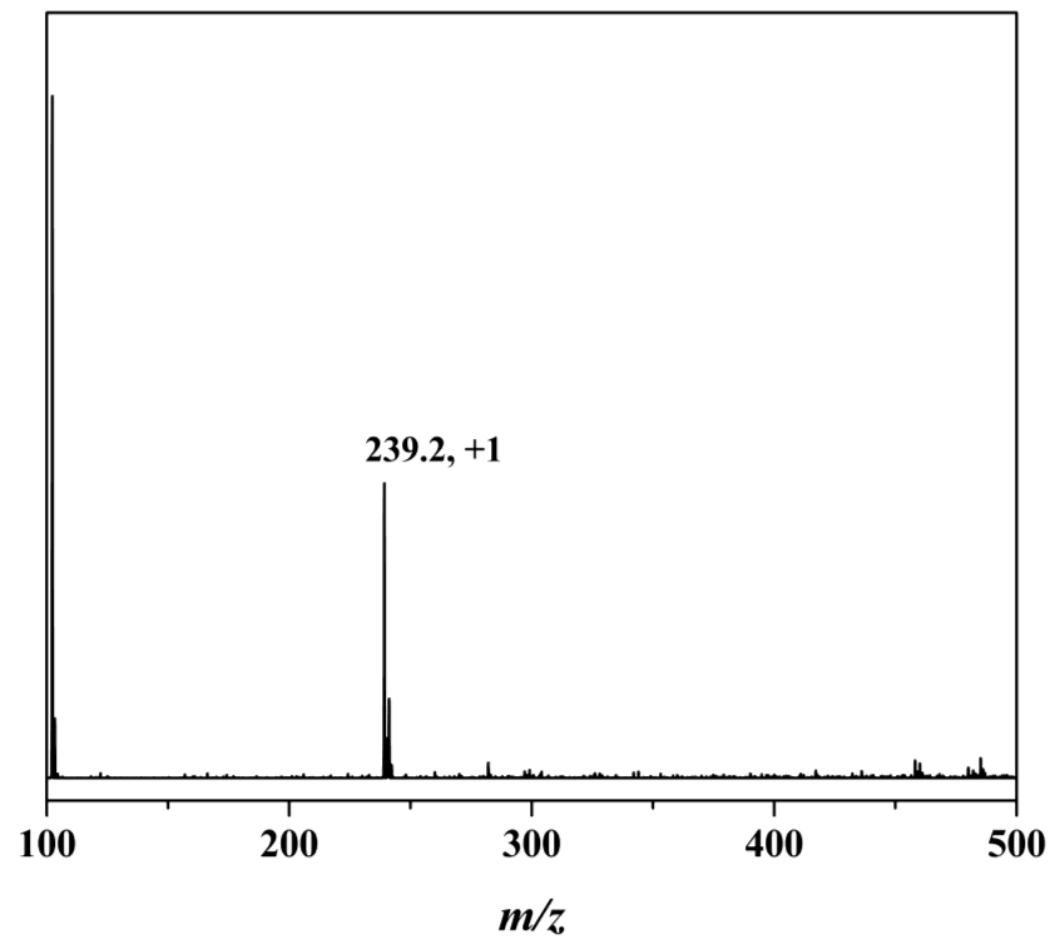

Fig. S24. Positive ion ESI-MS spectrum for DASAIND in $\mathrm{MeOH}$; where $m / z=239.2[\mathrm{M}+$ $\left.\mathrm{Na}^{+}\right]^{+}$.

ESI-MS spectra were predominantly consistent with either proton or $\mathrm{Na}+$ association for DASA precursors (Fig. S22-S23). Additionally, the lack of these fragments detected in the relevant spectrum (Fig. S24) for the potential DASA ligand was consistent with unsuccessful synthesis. 
S5. Attenuated Reflectance-Fourier Transform Infrared (ATR-FTIR) Spectroscopy

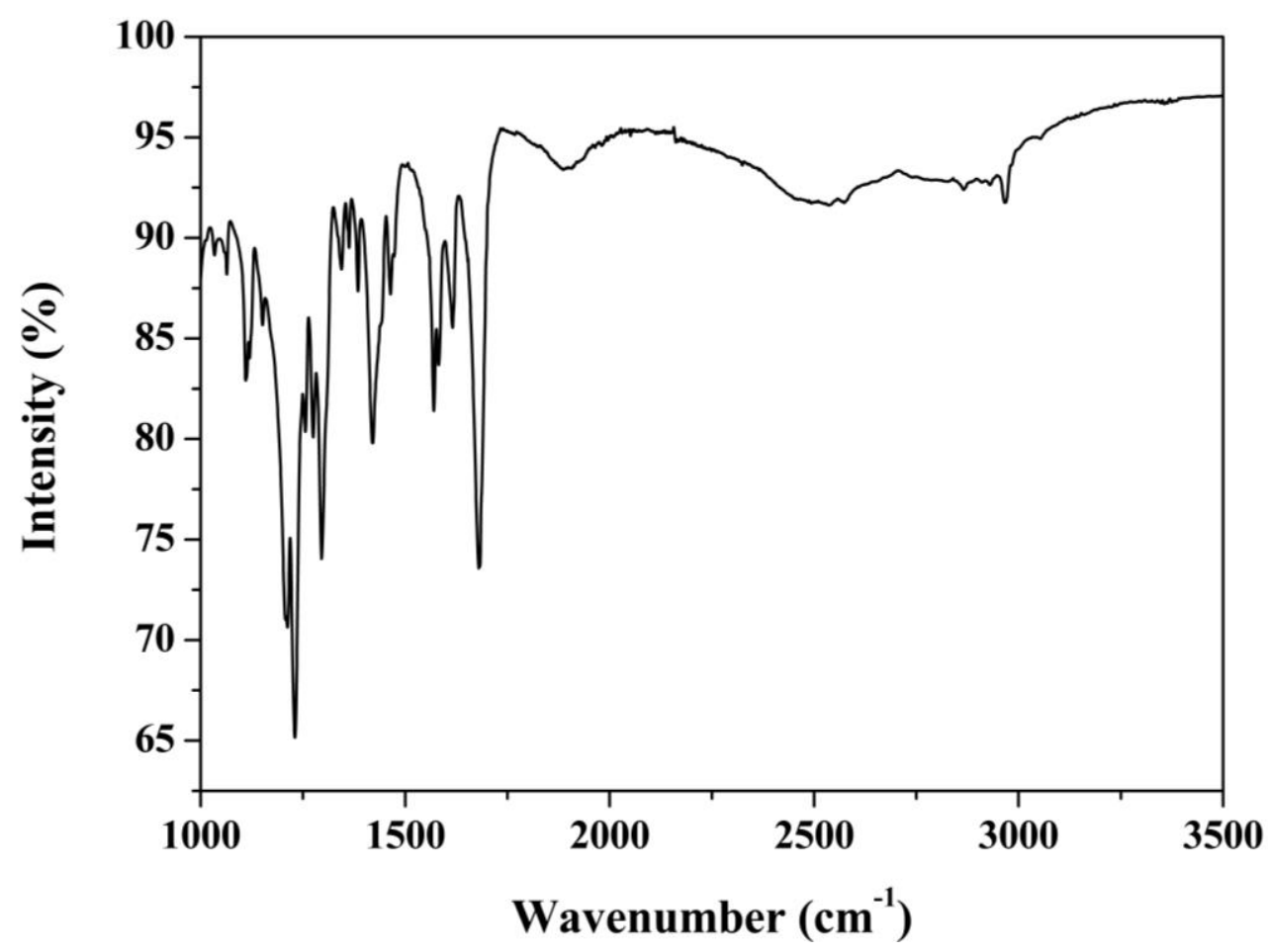

Fig. S25. ATR-FTIR spectrum for indoline from $1000-3500 \mathrm{~cm}^{-1}$.

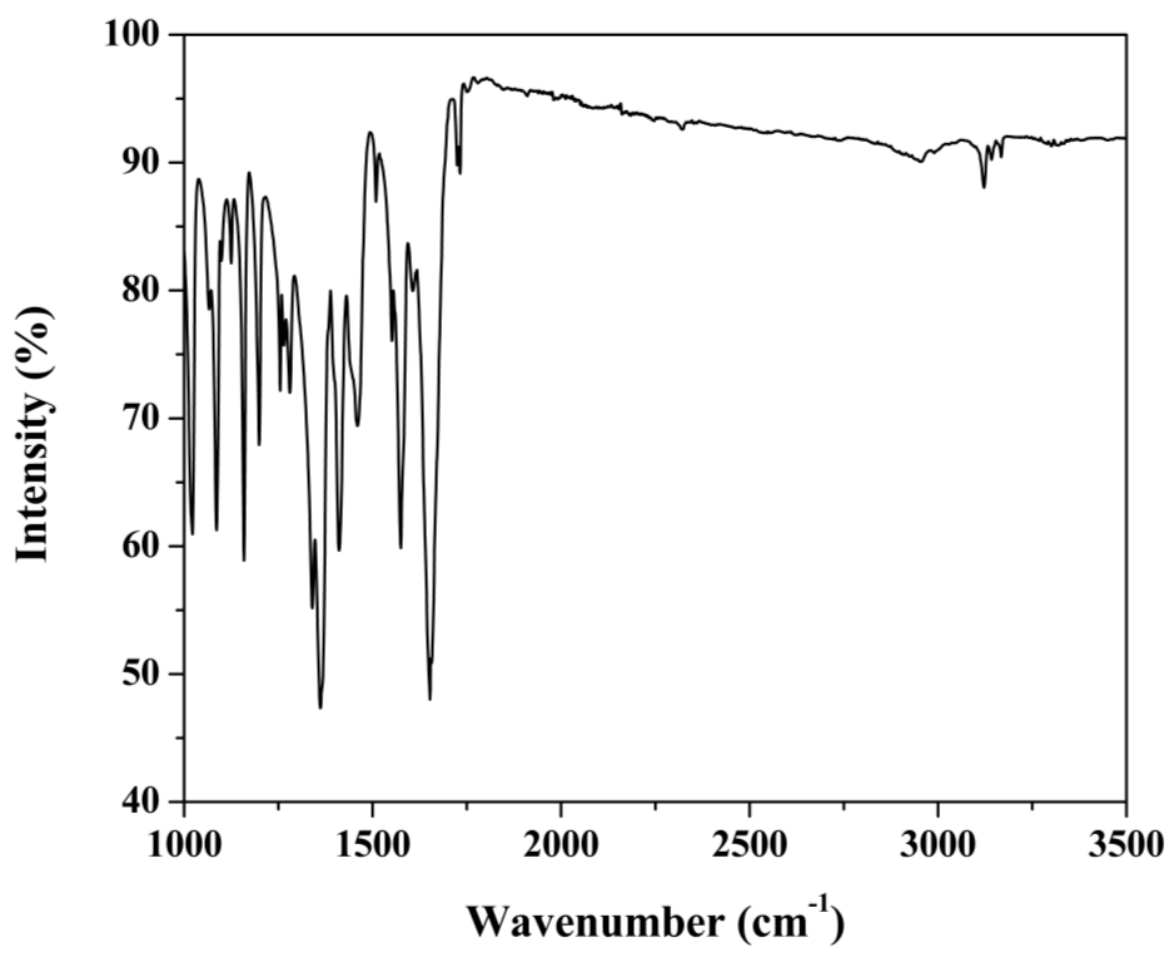

Fig. S26. ATR-FTIR spectrum for $\mathrm{Me}_{2} \mathrm{DP}$ from $1000-3500 \mathrm{~cm}^{-1}$. 


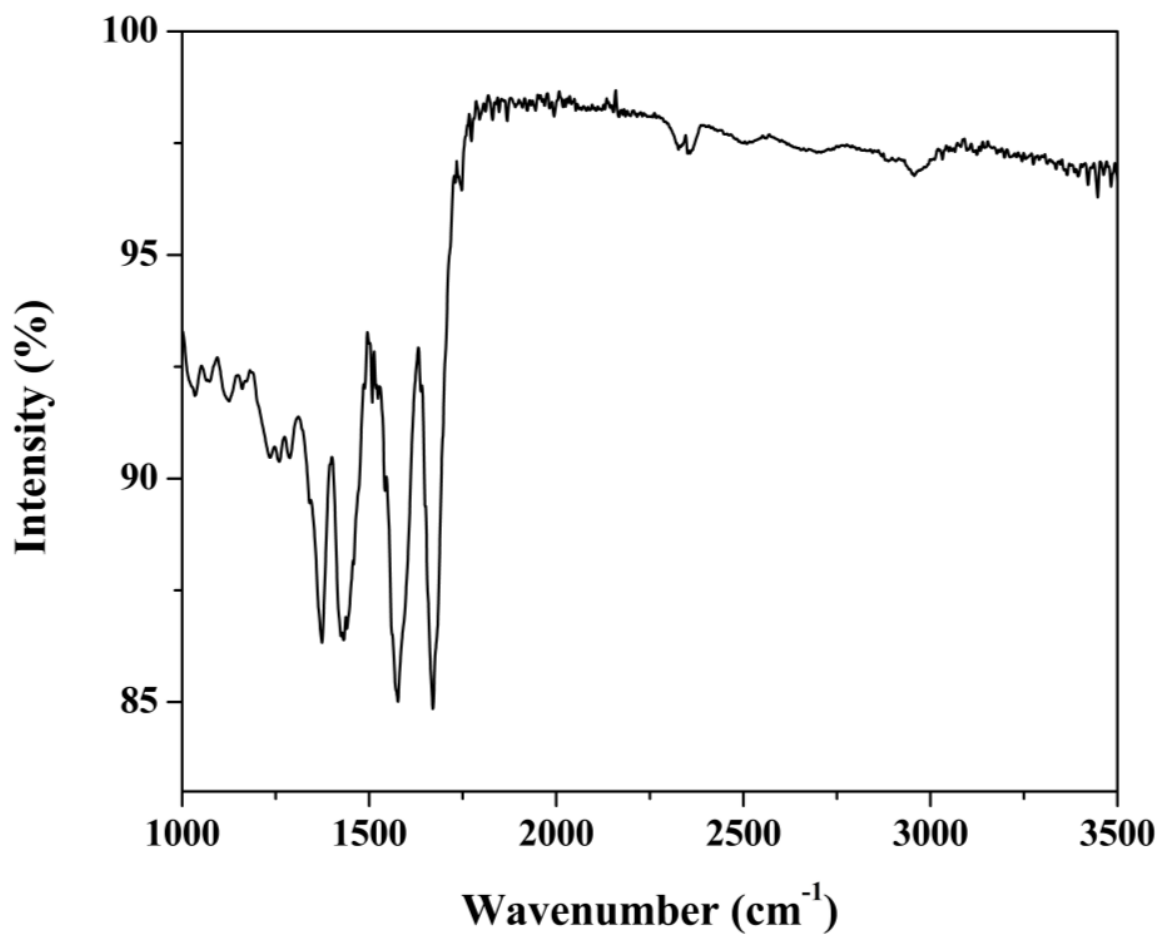

Fig. S27. ATR-FTIR spectrum for DASAIND from $1000-3500 \mathrm{~cm}^{-1}$.

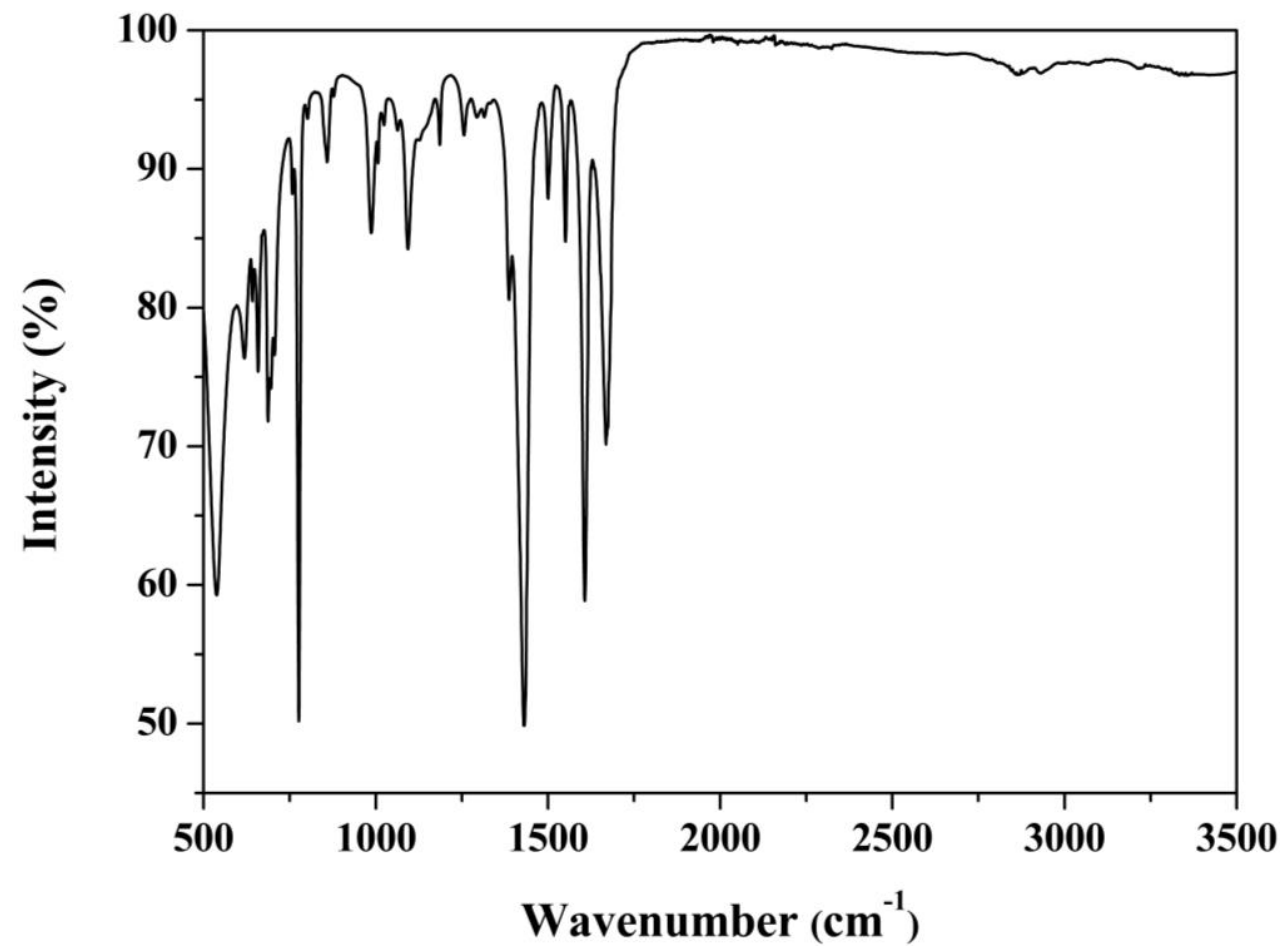

Fig. S28. ATR-FTIR spectrum for DUT-5 from $500-3500 \mathrm{~cm}^{-1}$. 


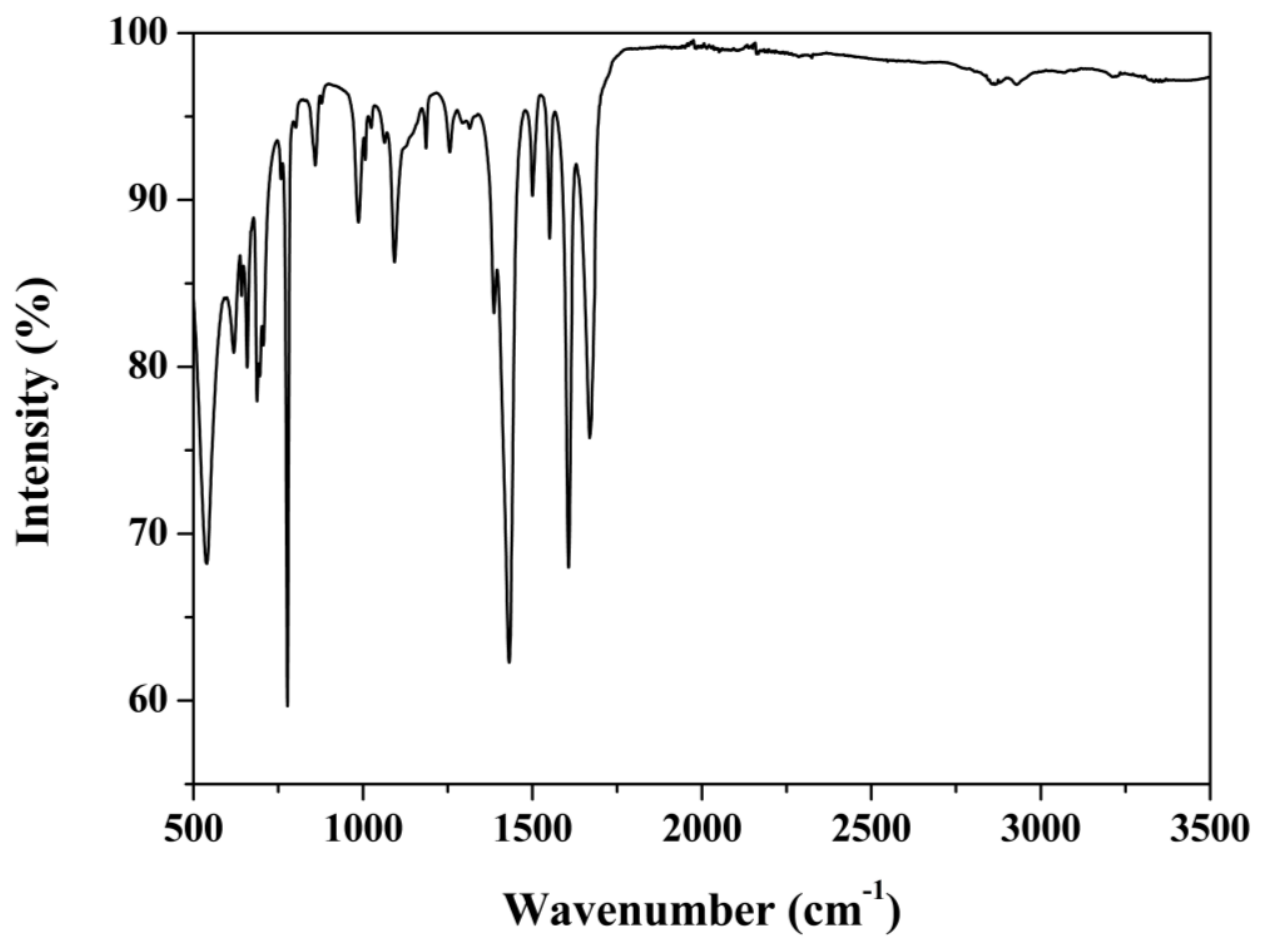

Fig. S29. ATR-FTIR spectrum for DUT-5(indoline) 0.5 from $500-3500 \mathrm{~cm}^{-1}$.

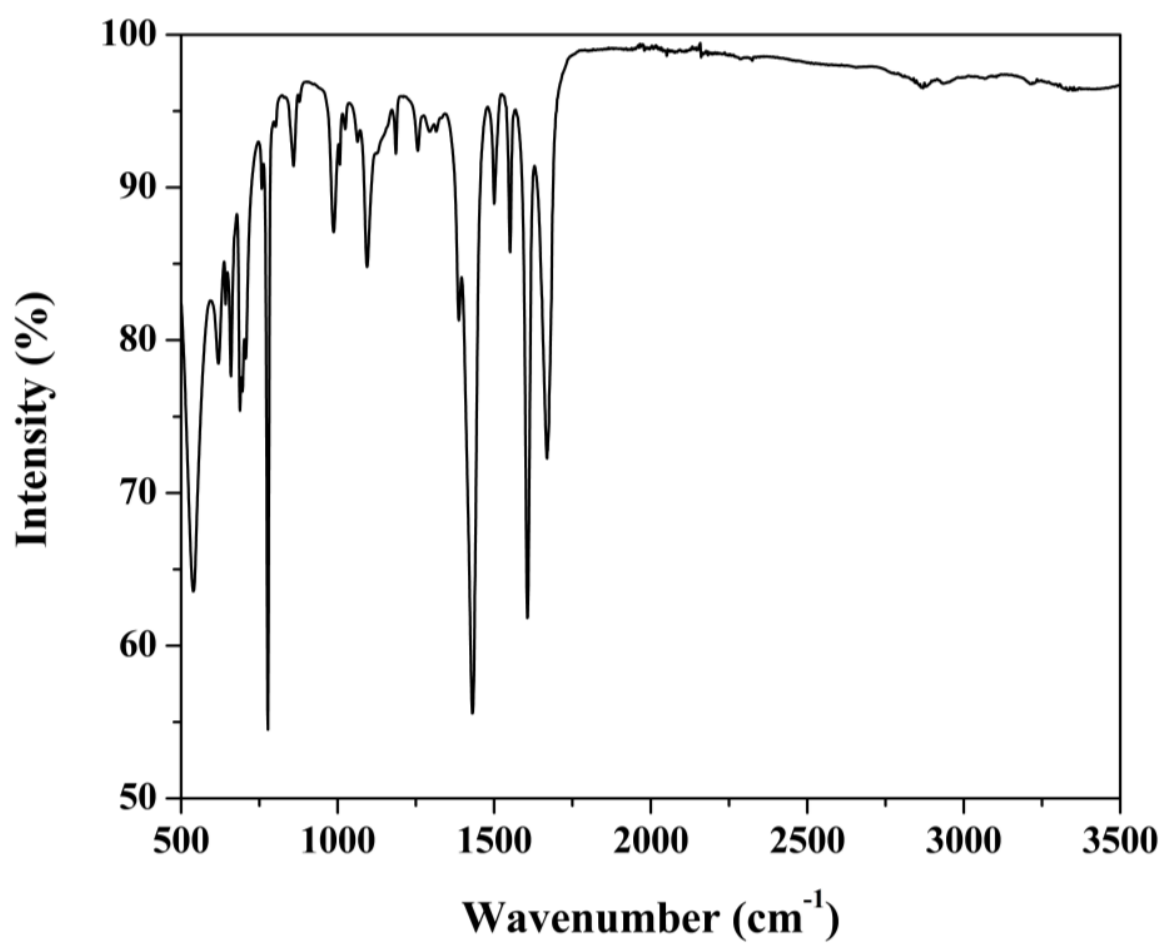

Fig. S30. ATR-FTIR spectrum for DUT-5(indoline) $)_{1.0}$ from $500-3500 \mathrm{~cm}^{-1}$. 


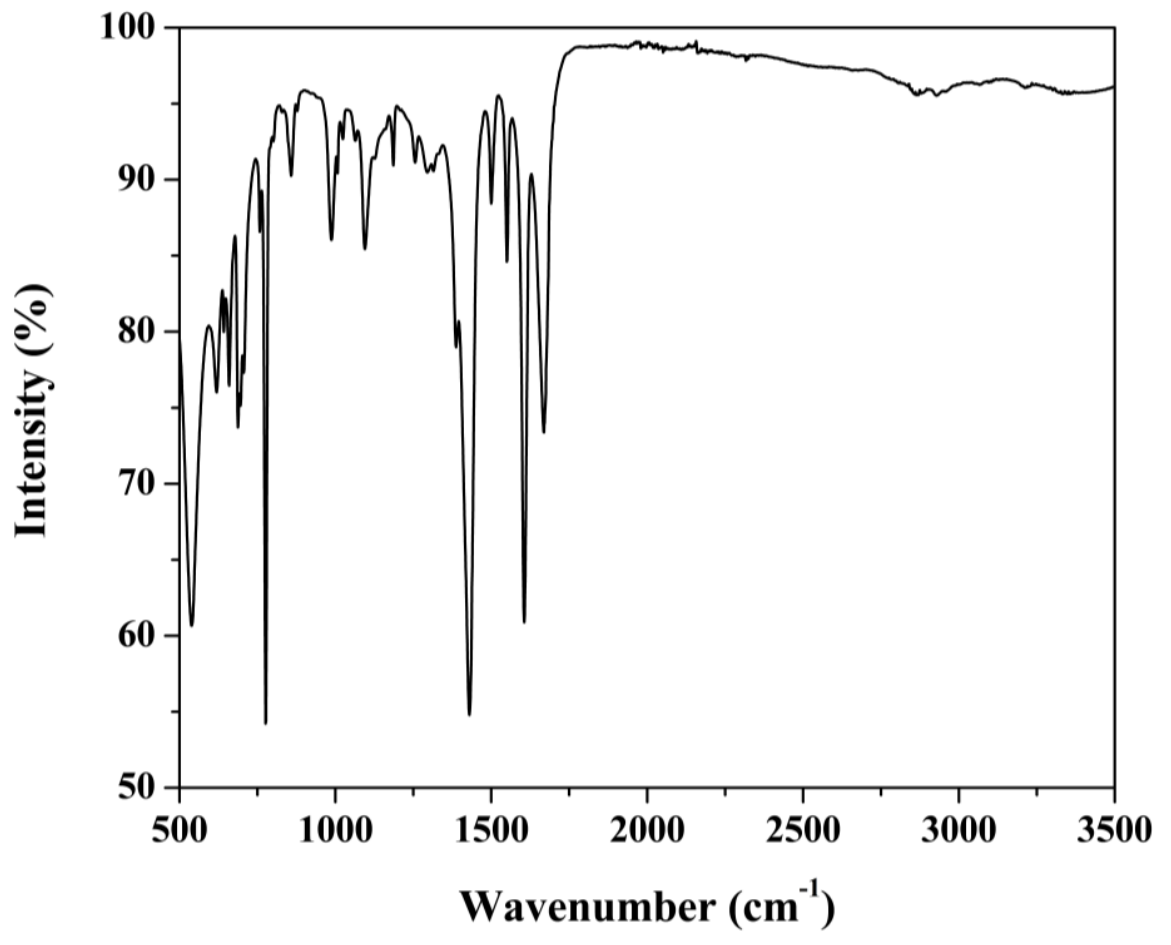

Fig. S31. ATR-FTIR spectrum for DUT-5(indoline) 1.5 from $500-3500 \mathrm{~cm}^{-1}$.

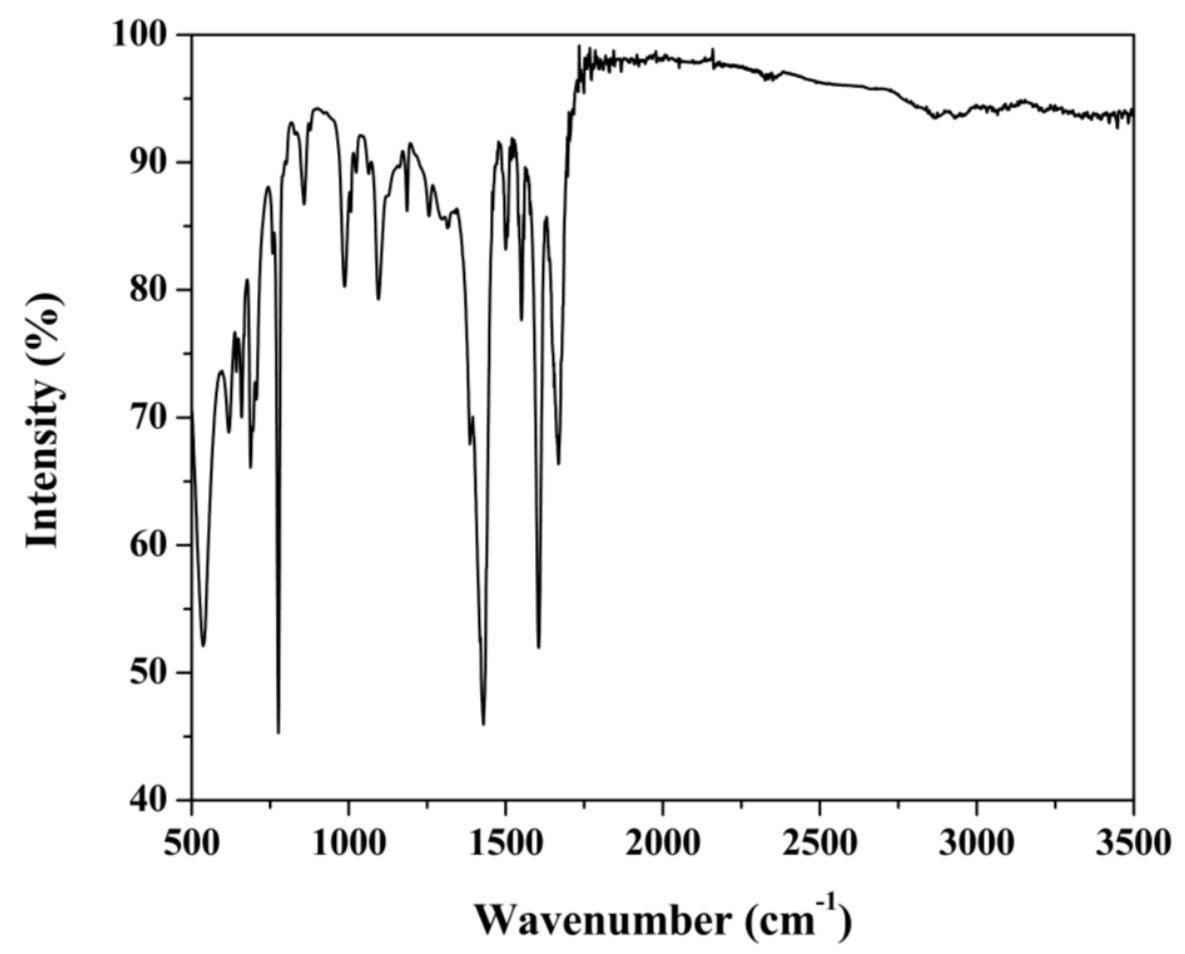

Fig. S32. ATR-FTIR spectrum for DUT-5(indoline) 2.0 from $500-3500 \mathrm{~cm}^{-1}$. 


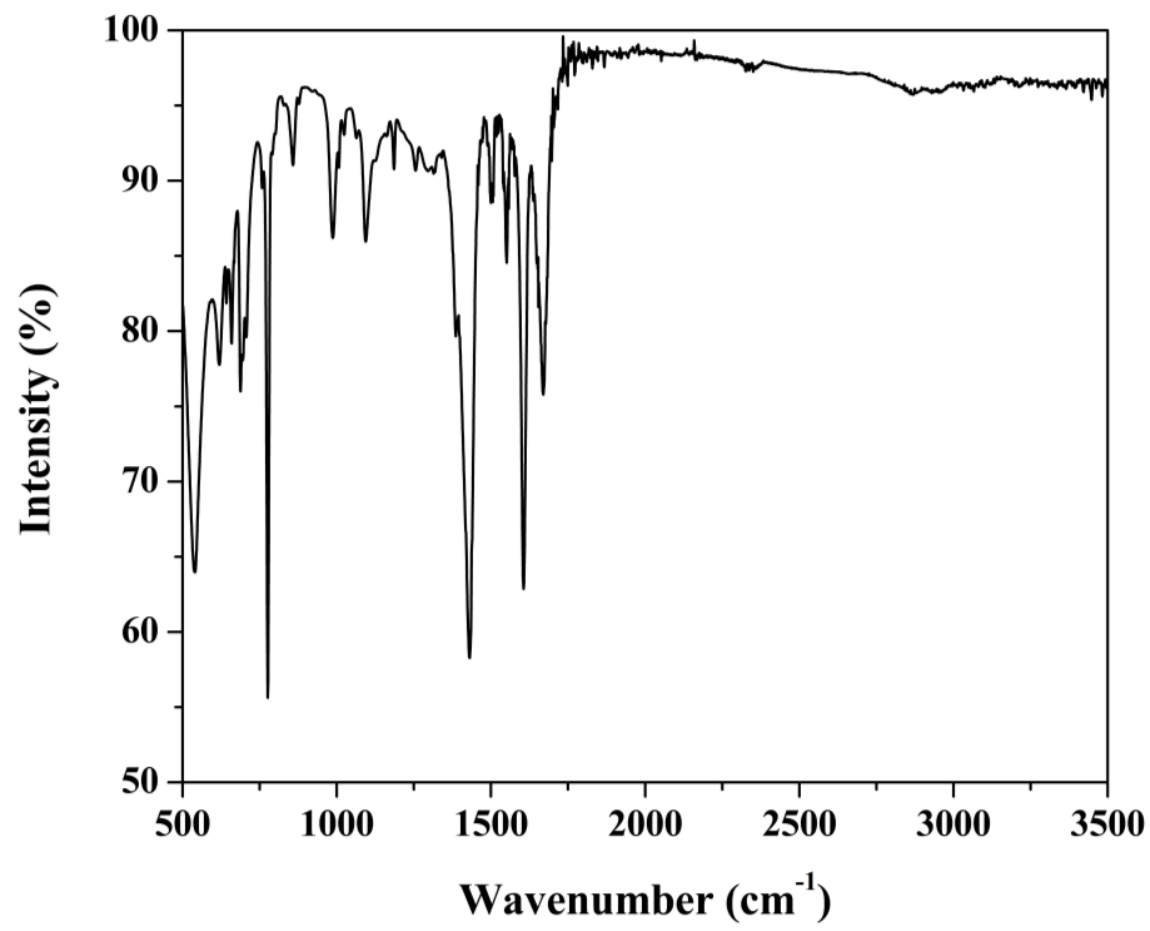

Fig. S33. ATR-FTIR spectrum for DUT-5(indoline) 2.5 from $500-3500 \mathrm{~cm}^{-1}$.

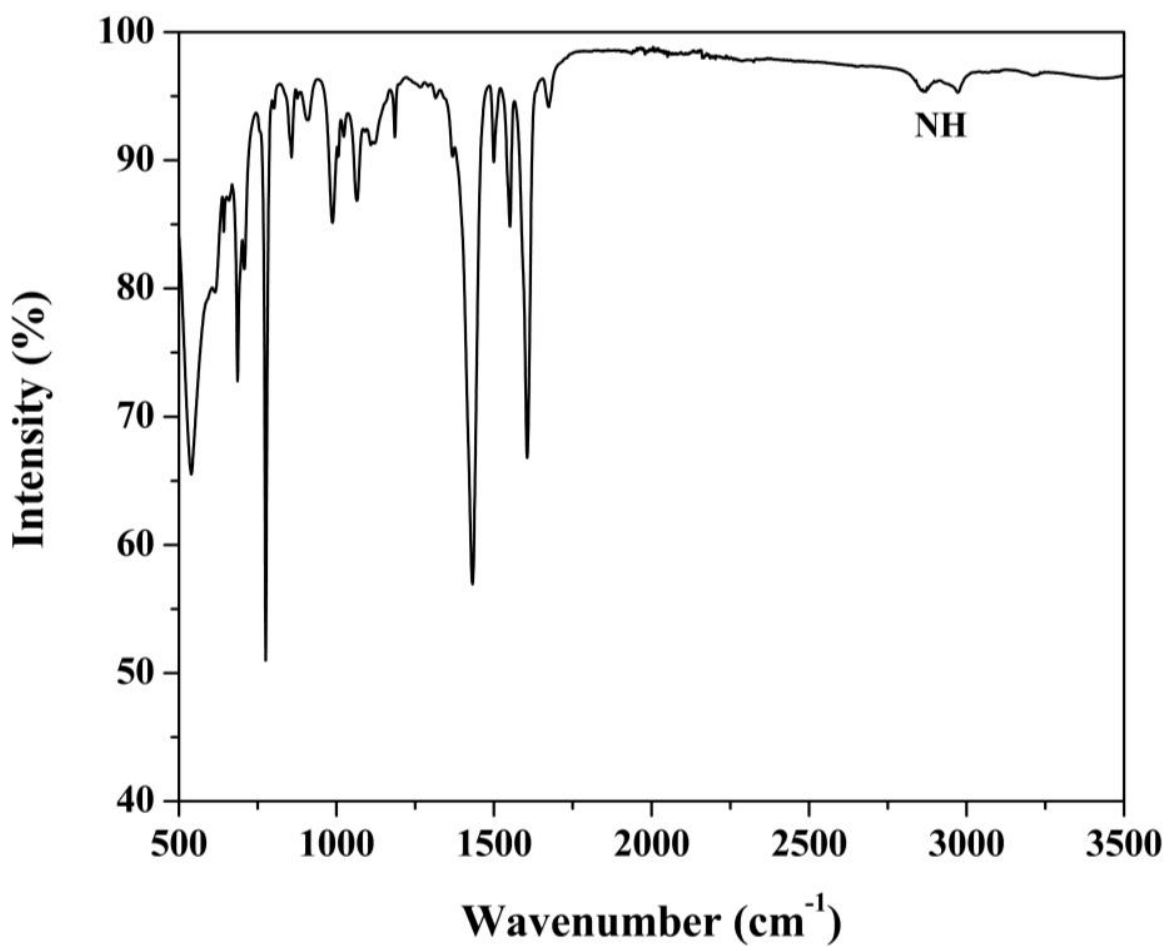

Fig. S34. ATR-FTIR spectrum for DUT-5(indoline) ${ }_{0.5}$ (DASA) from $500-3500 \mathrm{~cm}^{-1}$. 


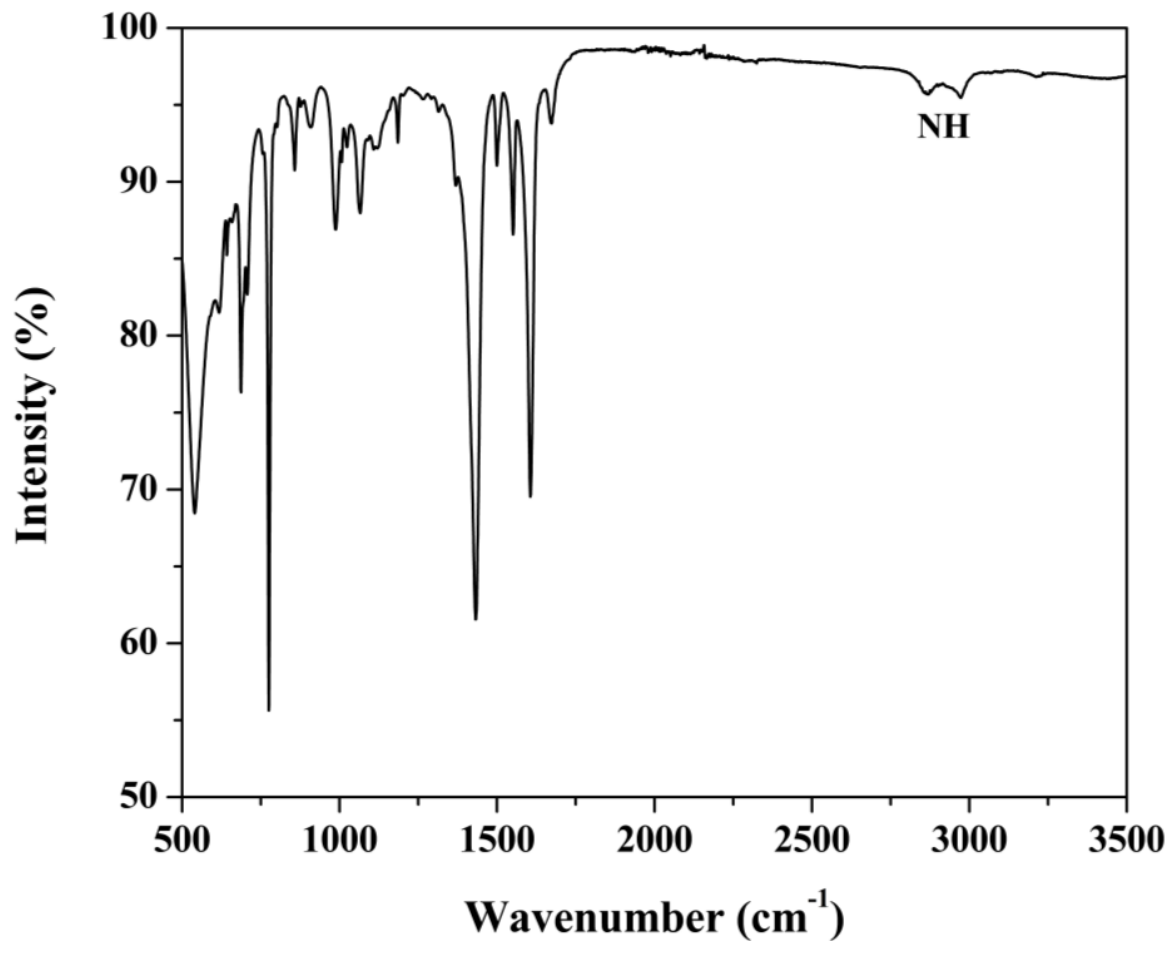

Fig. S35. ATR-FTIR spectrum for DUT-5(indoline) ${ }_{1.0}(\mathrm{DASA})$ from $500-3500 \mathrm{~cm}^{-1}$.

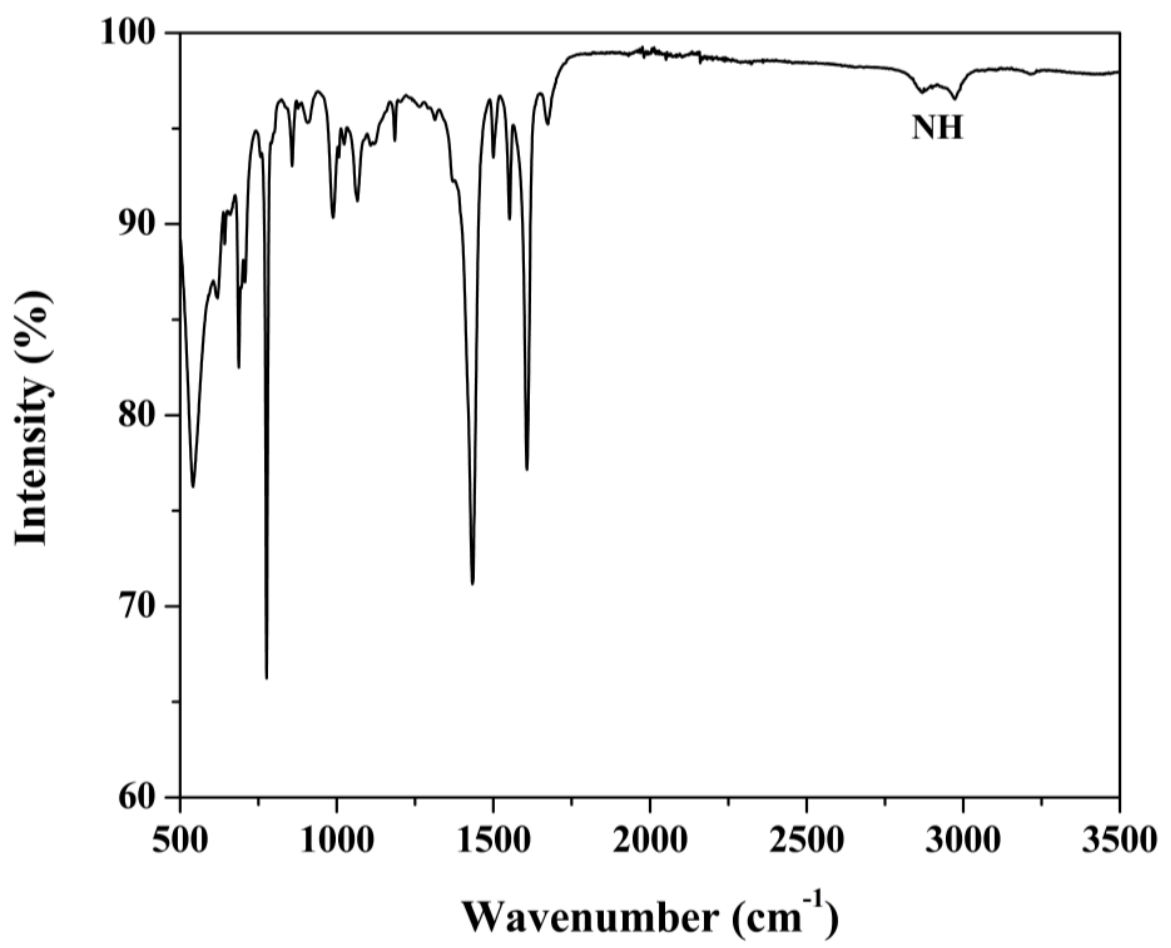

Fig. S36. ATR-FTIR spectrum for DUT-5(indoline) ${ }_{1.5}$ (DASA) from 500-3500 $\mathrm{cm}^{-1}$. 


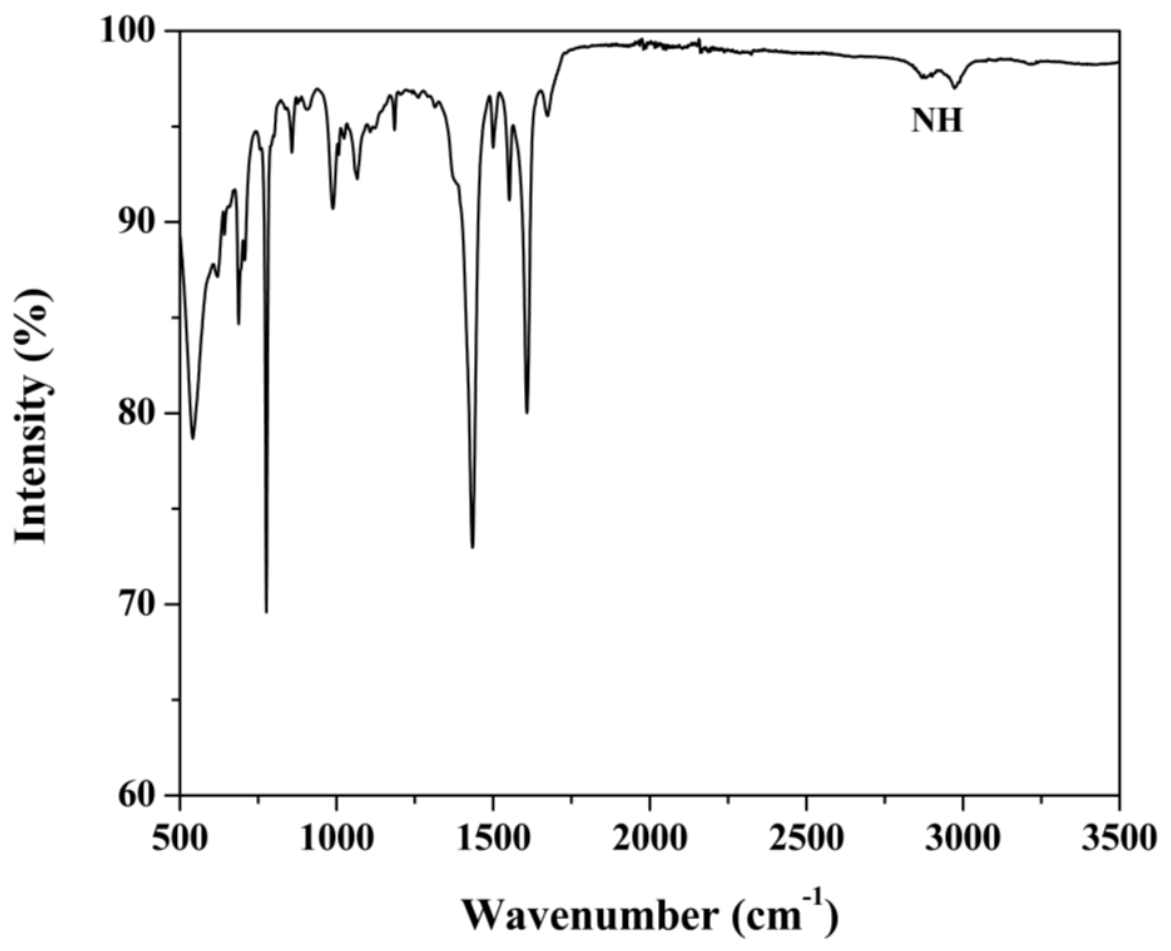

Fig. S37. ATR-FTIR spectrum for DUT-5(indoline) ${ }_{2.0}(\mathrm{DASA})$ from $500-3500 \mathrm{~cm}^{-1}$.

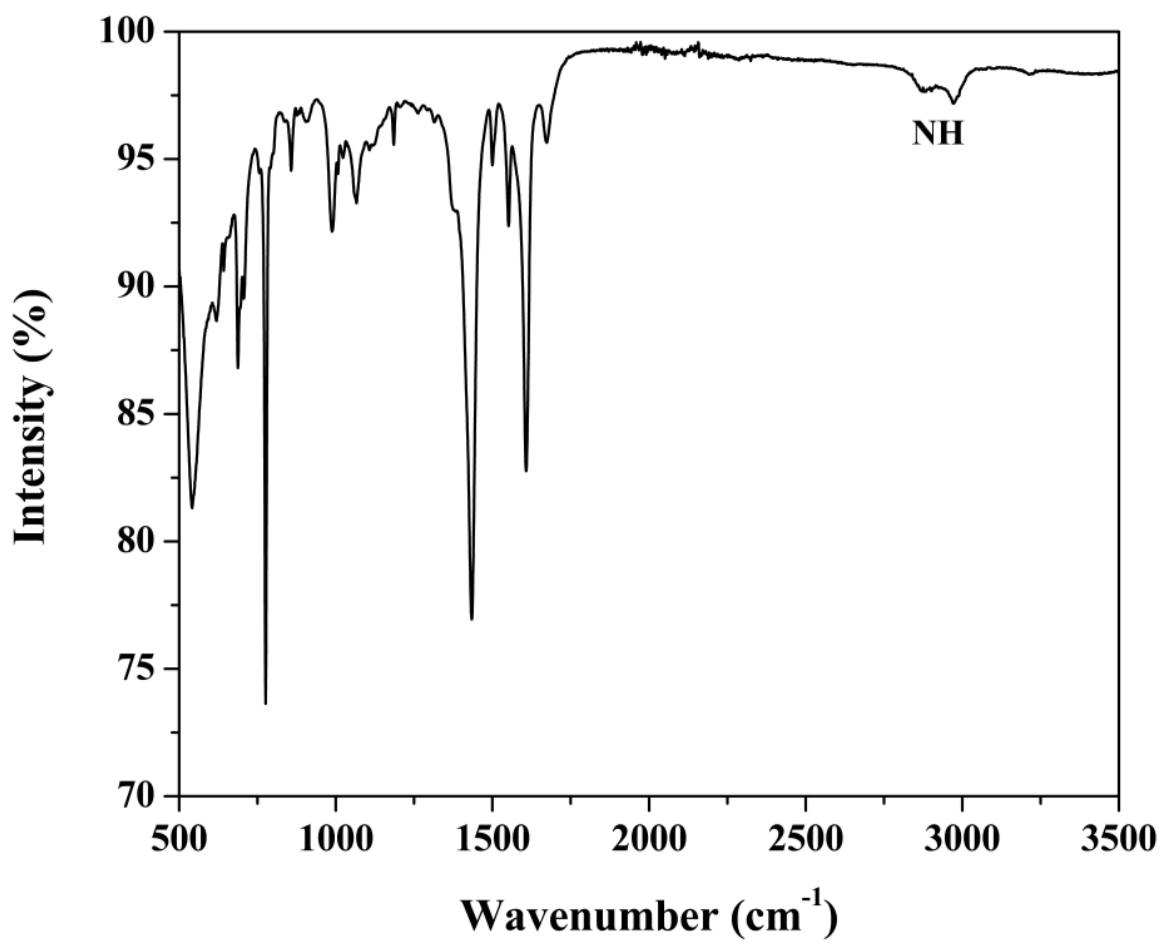

Fig. S38. ATR-FTIR spectrum for DUT-5(indoline) ${ }_{2.5}$ (DASA) from 500-3500 $\mathrm{cm}^{-1}$. 


\section{S6. Ultraviolet-Visible (UV-vis) Spectroscopy}

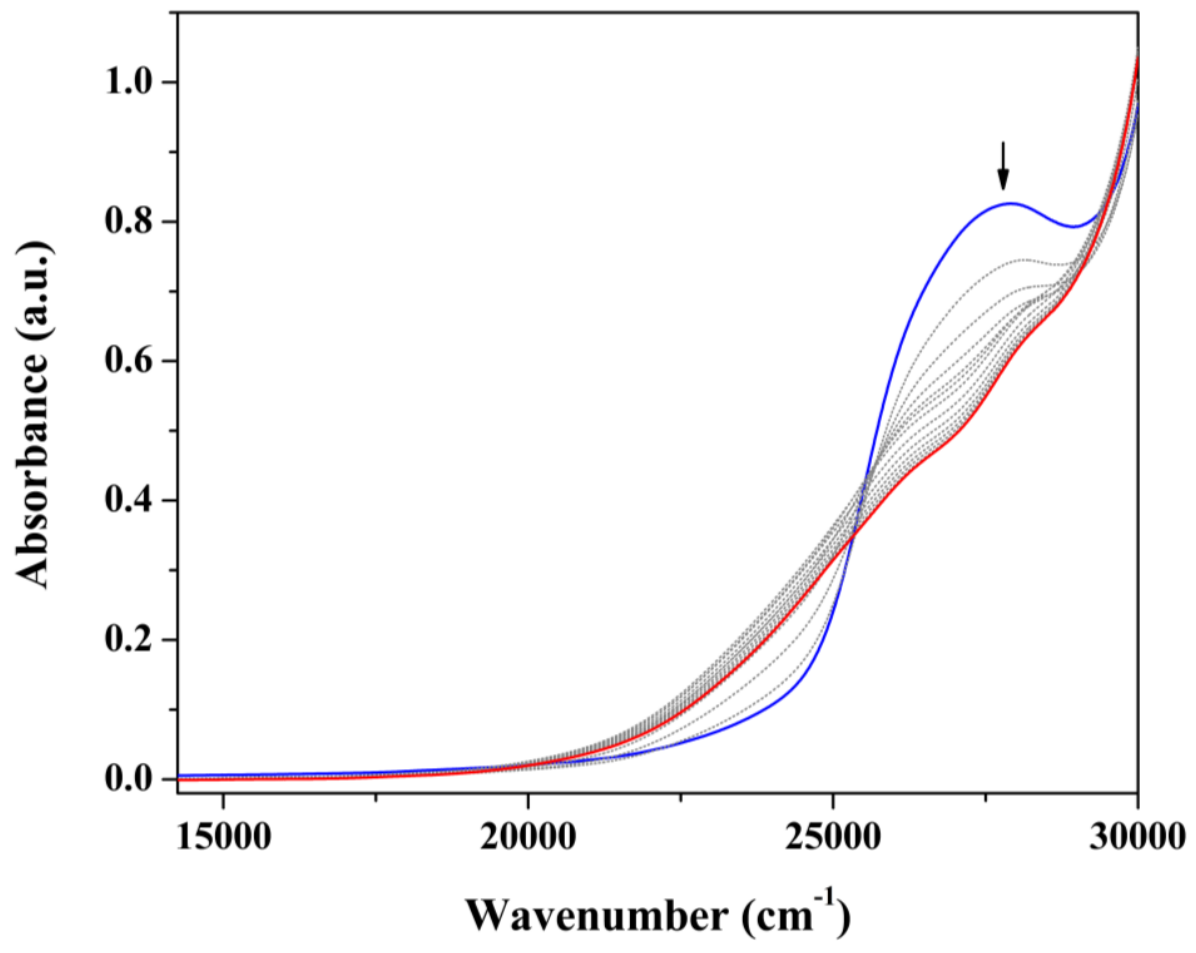

Fig. S39. Solution UV-vis spectra from $14250-30000 \mathrm{~cm}^{-1}$ for DASAIND reaction mixture recorded at 90 minute intervals over a 20 hour period.

Time-course reaction UV-vis spectra (Fig. S39) for the prospective DASAIND reaction solutions indicated a spectral change over 20 hours. Variation in the spectrum was in the form of a band at $27920 \mathrm{~cm}^{-1}(358 \mathrm{~nm})$ which disappeared during the reaction. Further evidence that the DASA could not be prepared as a discrete compound is the lack of a transition in the visible region which would be consistent with the $\mathrm{S}_{0}-\mathrm{S}_{1}$ of the DASA triene chain. ${ }^{4,5}$ 


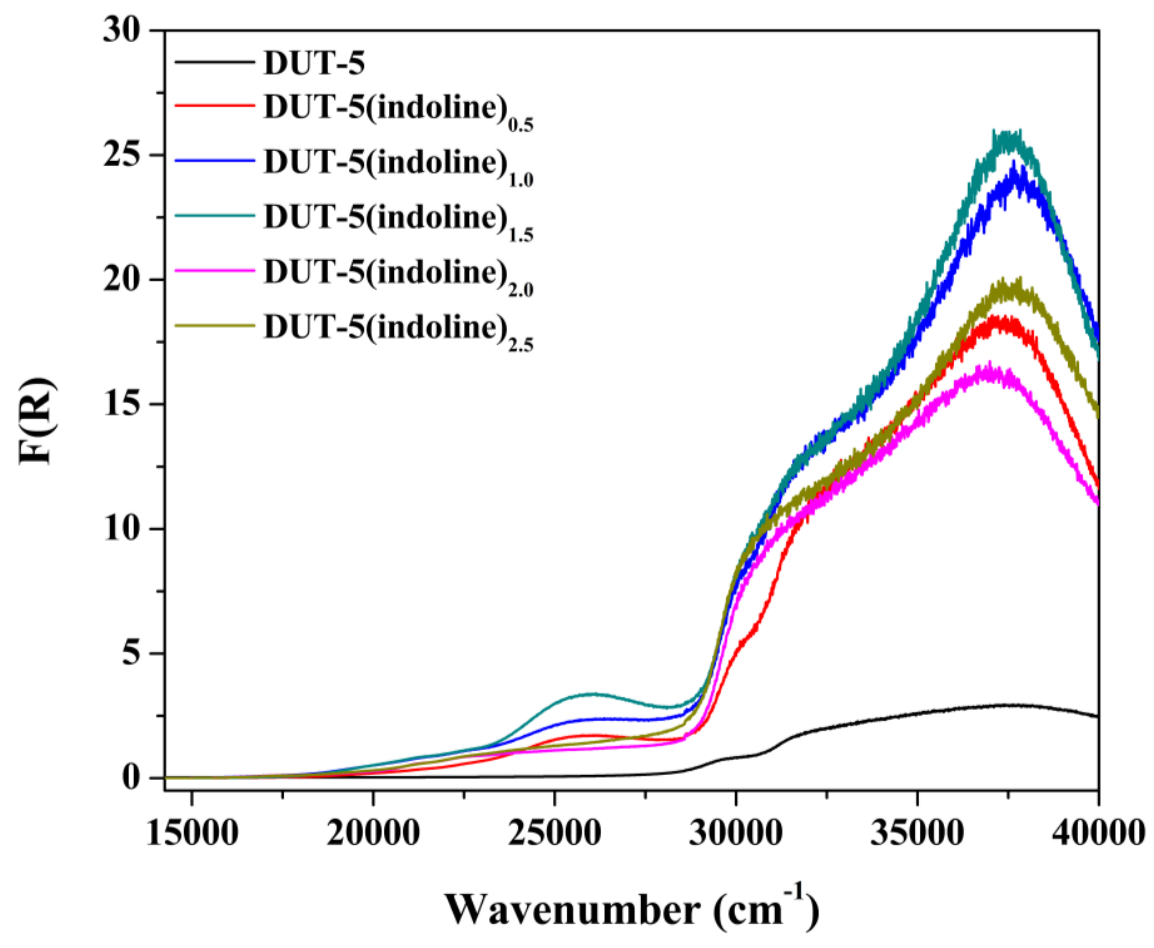

Fig. S40. Solid-state UV-vis spectra from $14250-40000 \mathrm{~cm}^{-1}$ for DUT-5(indoline) $0.5-2.5$.

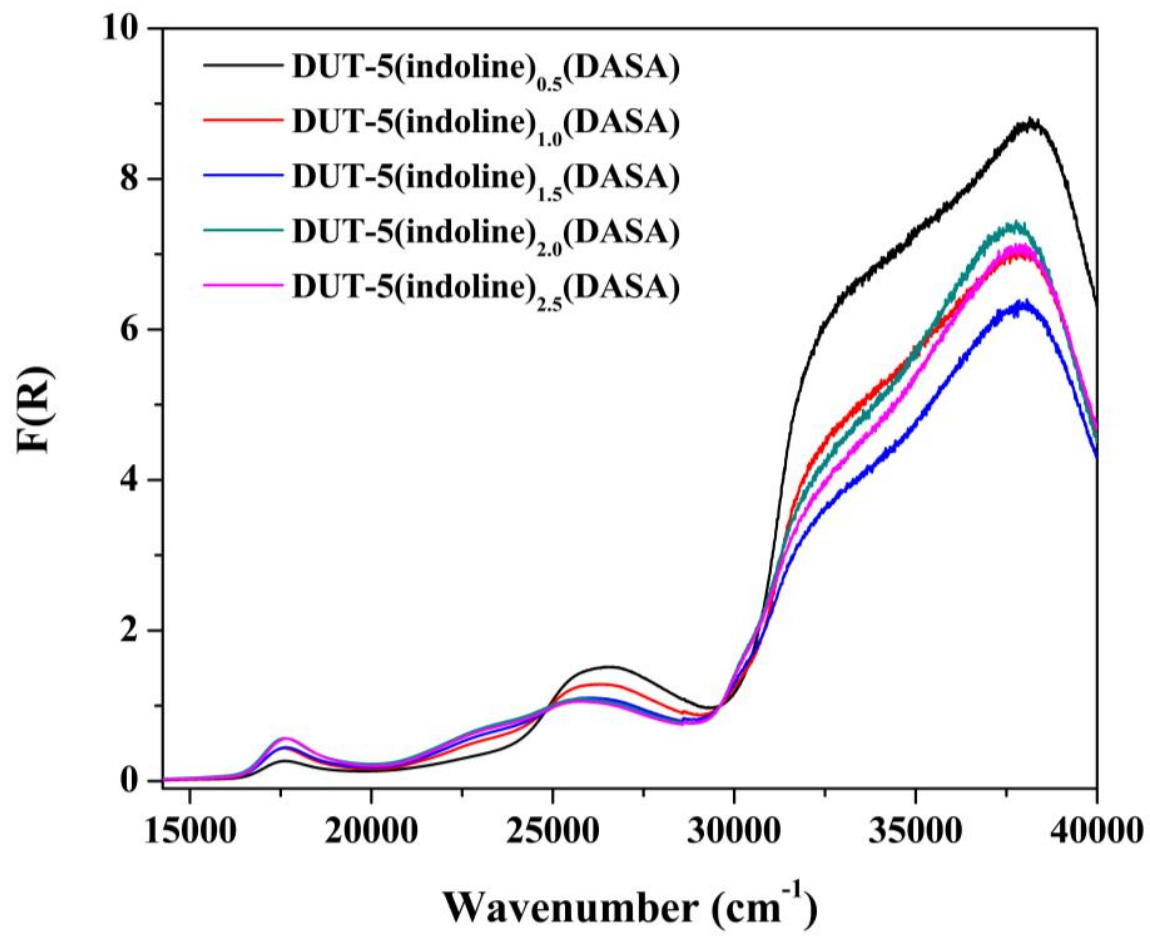

Fig. S41. Solid-state UV-vis spectra from 14250-40000 $\mathrm{cm}^{-1}$ for DUT-5(indoline)0.52.5(DASA). 


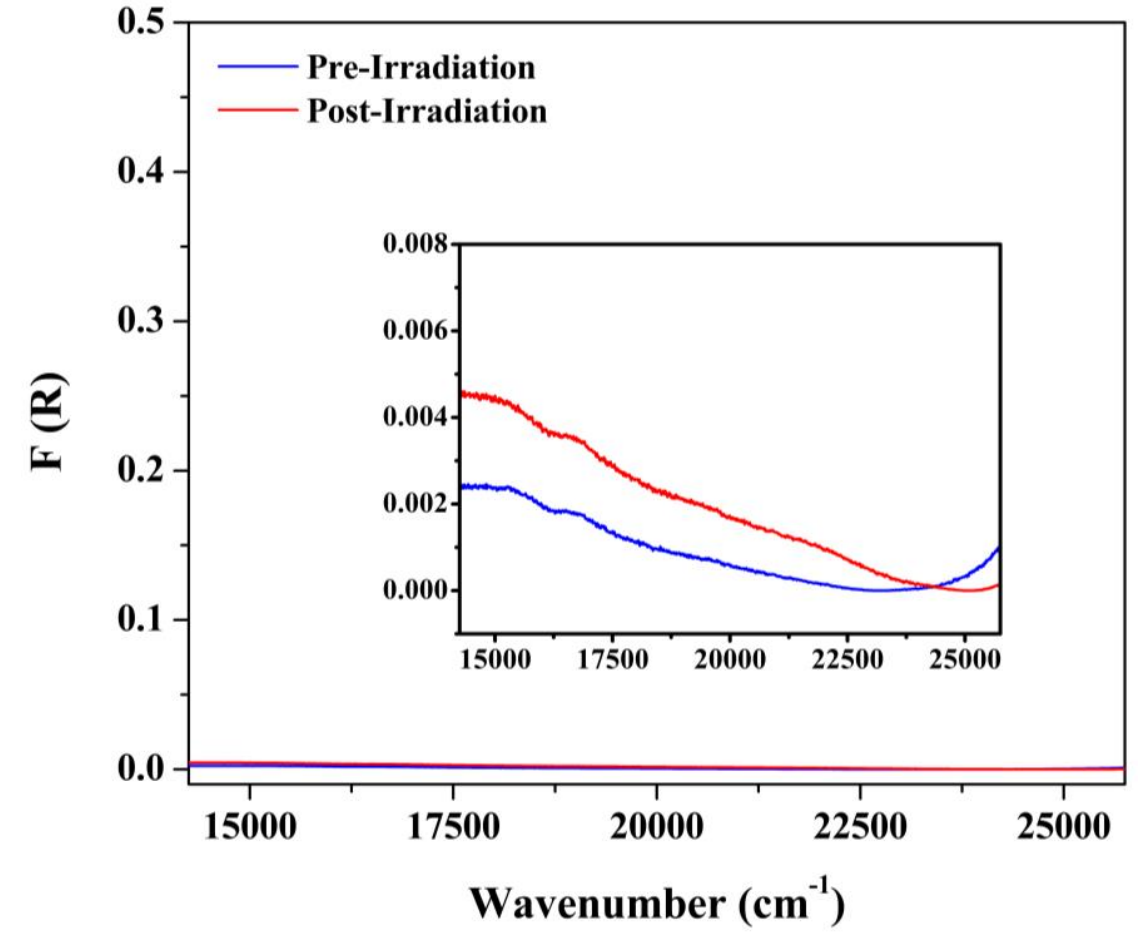

Fig. S42. Solid-state UV-vis spectra from $14250-25750 \mathrm{~cm}^{-1}$ for DUT-5, where the MOF was irradiated with white light for 20 minutes.

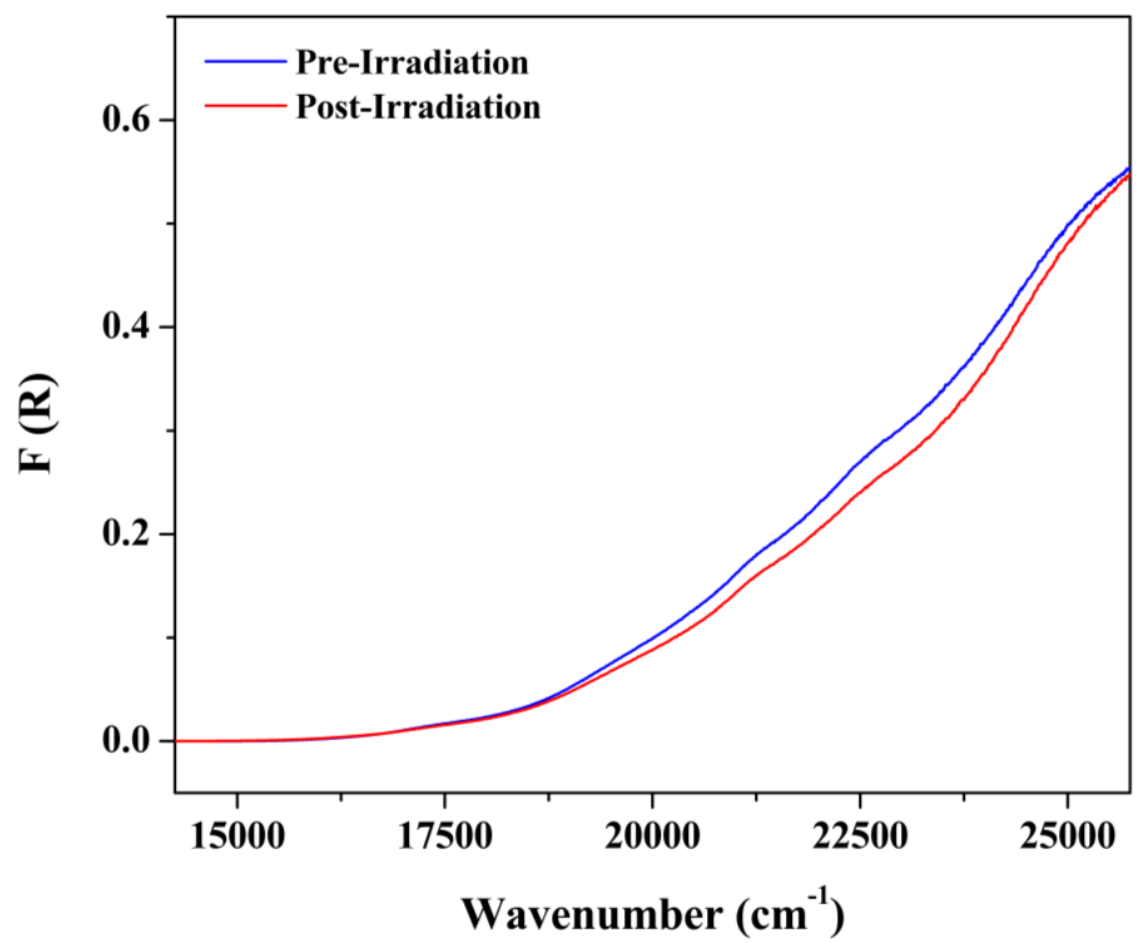

Fig. S43. Solid-state UV-vis spectra from $14250-25750 \mathrm{~cm}^{-1}$ for DUT-5(indoline) 0.5 , where the MOF was irradiated with white light for 40 minutes. 


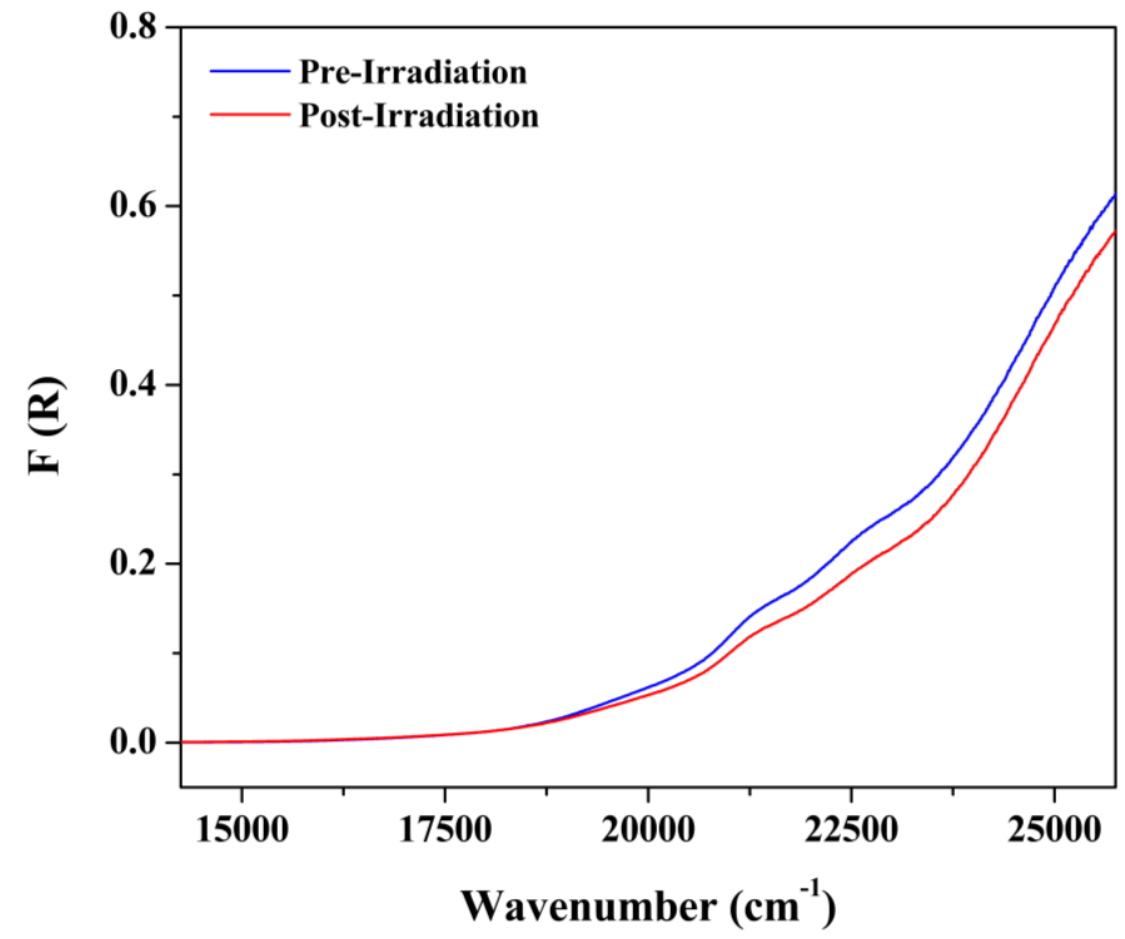

Fig. S44. Solid-state UV-vis spectra from $14250-25750 \mathrm{~cm}^{-1}$ for DUT-5(indoline) 1.0 , where the MOF was irradiated with white light for 20 minutes.

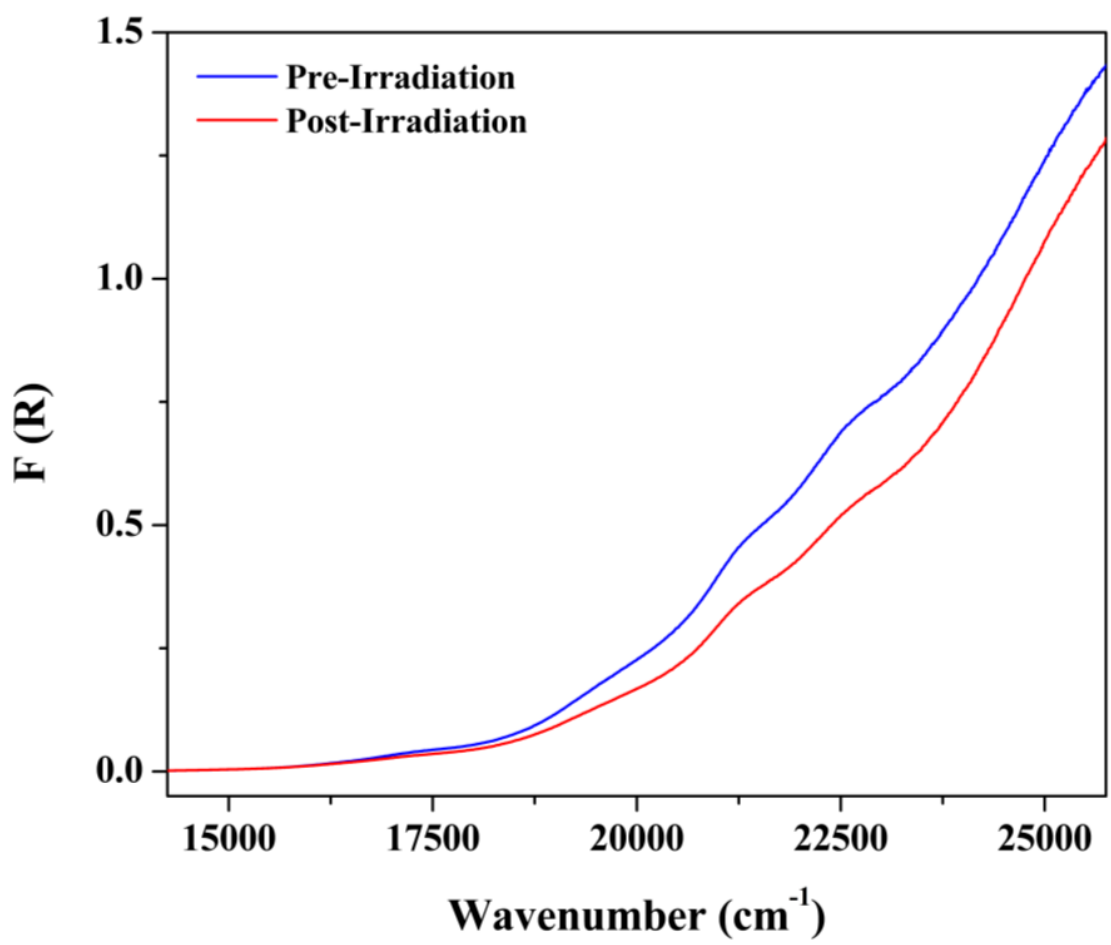

Fig. S45. Solid-state UV-vis spectra from $14250-25750 \mathrm{~cm}^{-1}$ for DUT-5(indoline) ${ }_{1.5}$, where the MOF was irradiated with white light for 40 minutes. 


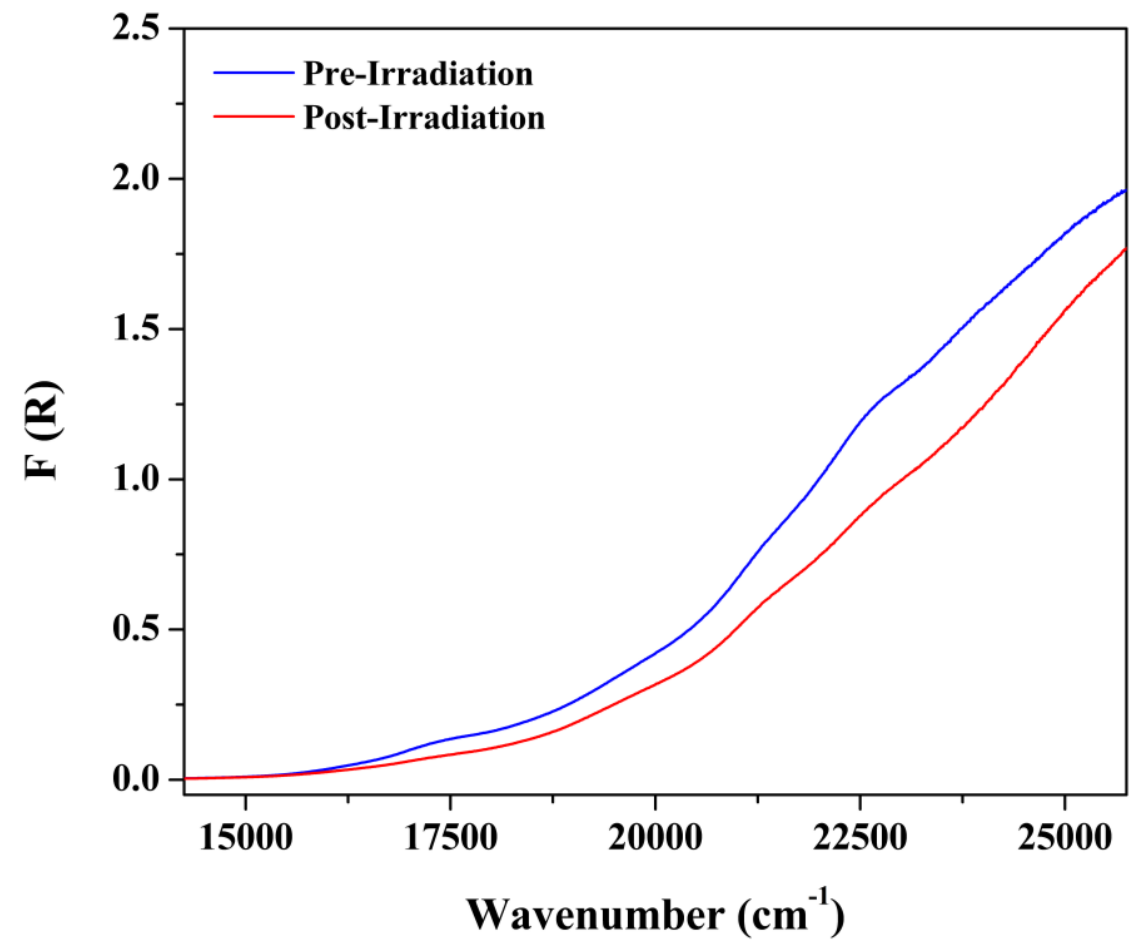

Fig. S46. Solid-state UV-vis spectra from $14250-25750 \mathrm{~cm}^{-1}$ for DUT-5(indoline) 2.0 , where the MOF was irradiated with white light for 40 minutes.

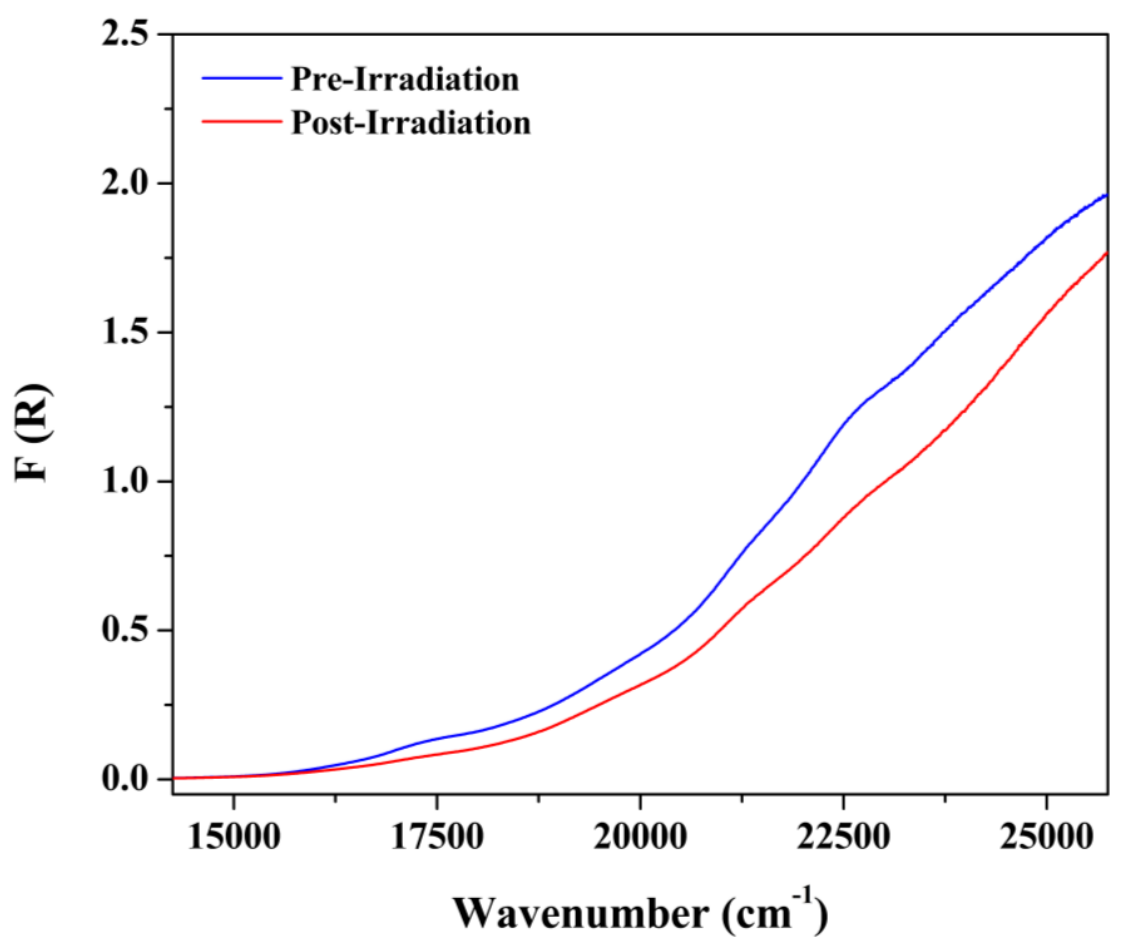

Fig. S47. Solid-state UV-vis spectra from $14250-25750 \mathrm{~cm}^{-1}$ for DUT-5(indoline) 2.5 , where the MOF was irradiated with white light for 40 minutes. 

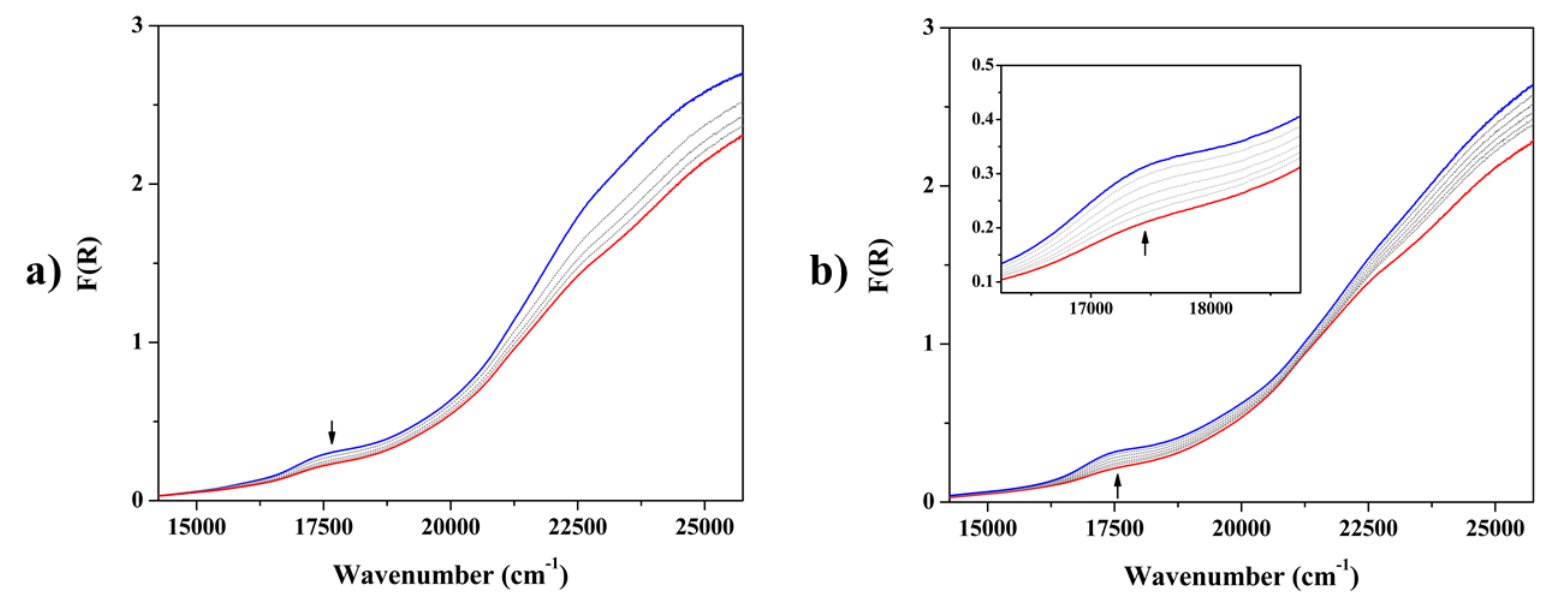

Fig. S48. Solid-state UV-vis spectra from $14250-25750 \mathrm{~cm}^{-1}$ for DUT-5(indoline) ${ }_{1.0}$ (DASA), where the MOF was irradiated with white light up to 80 minutes (a) and heated to $100^{\circ} \mathrm{C}(\mathrm{b})$.
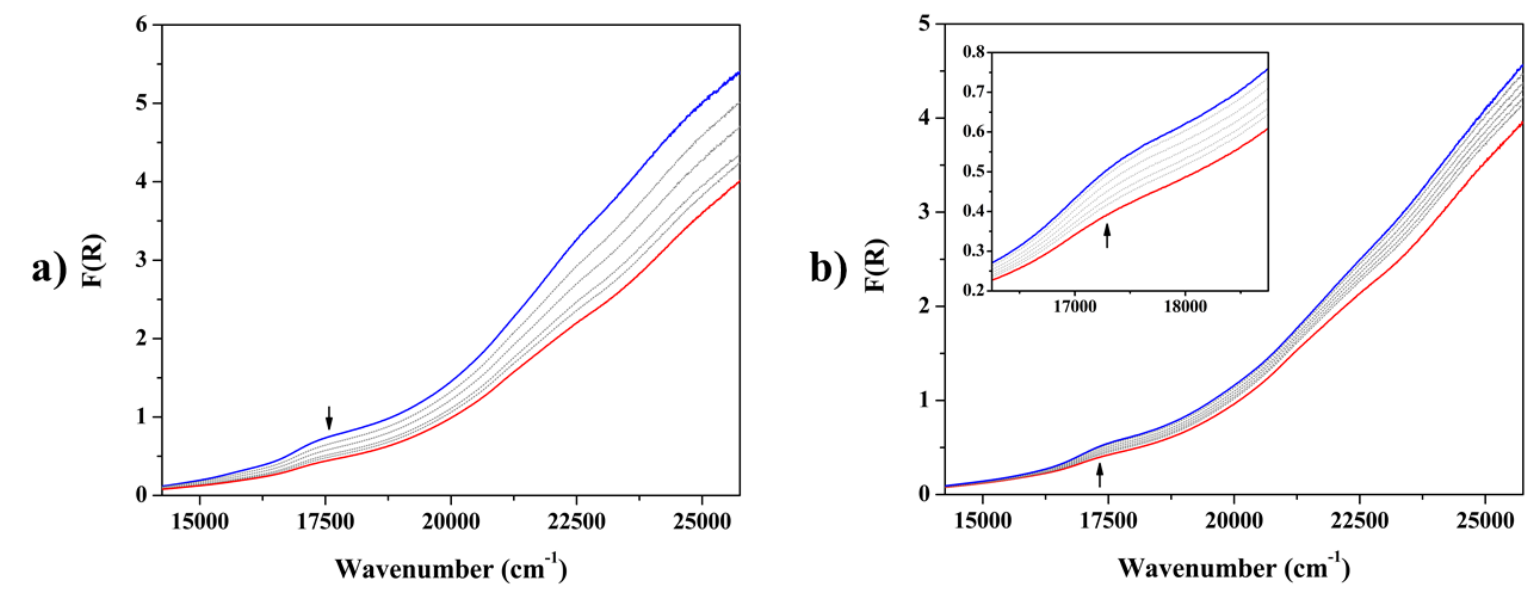

Fig. S49. Solid-state UV-vis spectra from $14250-25750 \mathrm{~cm}^{-1}$ for DUT-5(indoline) 1.5 (DASA), where the MOF was irradiated with white light up to 100 minutes (a) and heated to $100^{\circ} \mathrm{C} \mathrm{(b)}$.
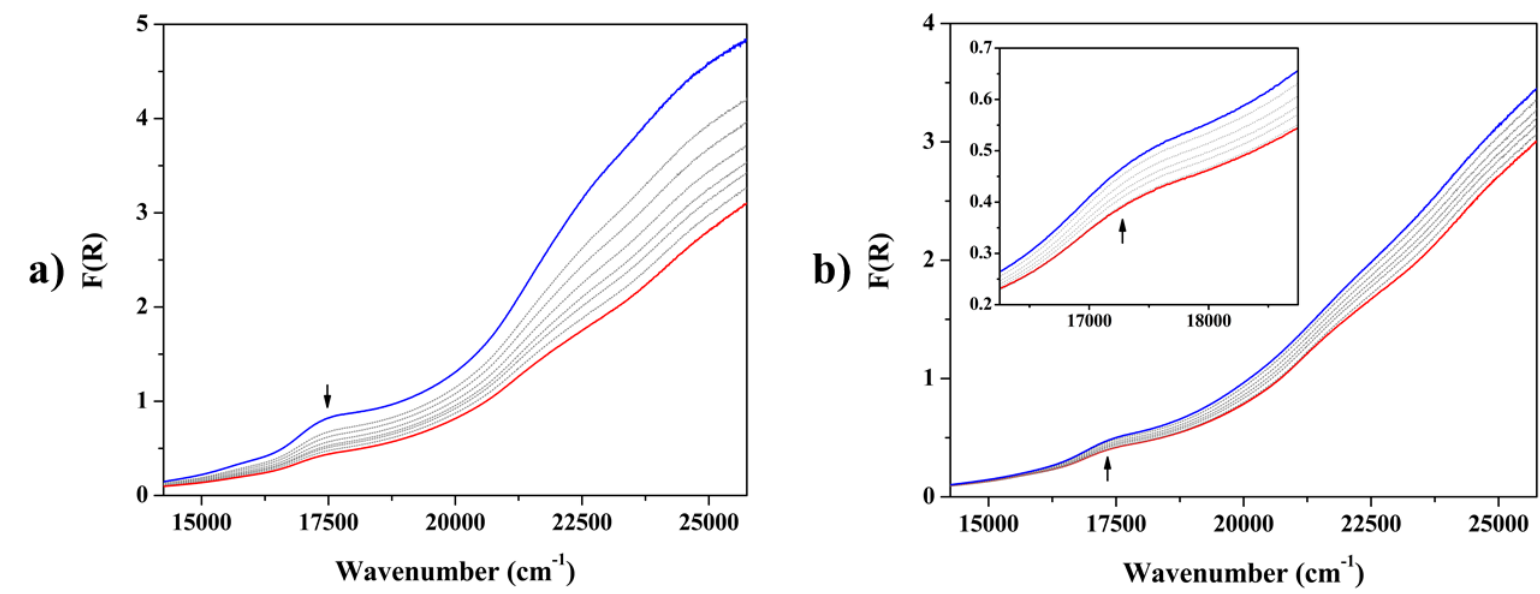

Fig. S50. Solid-state UV-vis spectra from $14250-25750 \mathrm{~cm}^{-1}$ for DUT-5(indoline) 2.0 (DASA), where the MOF was irradiated with white light up to 140 minutes (a) and heated to $100^{\circ} \mathrm{C} \mathrm{(b)}$. 

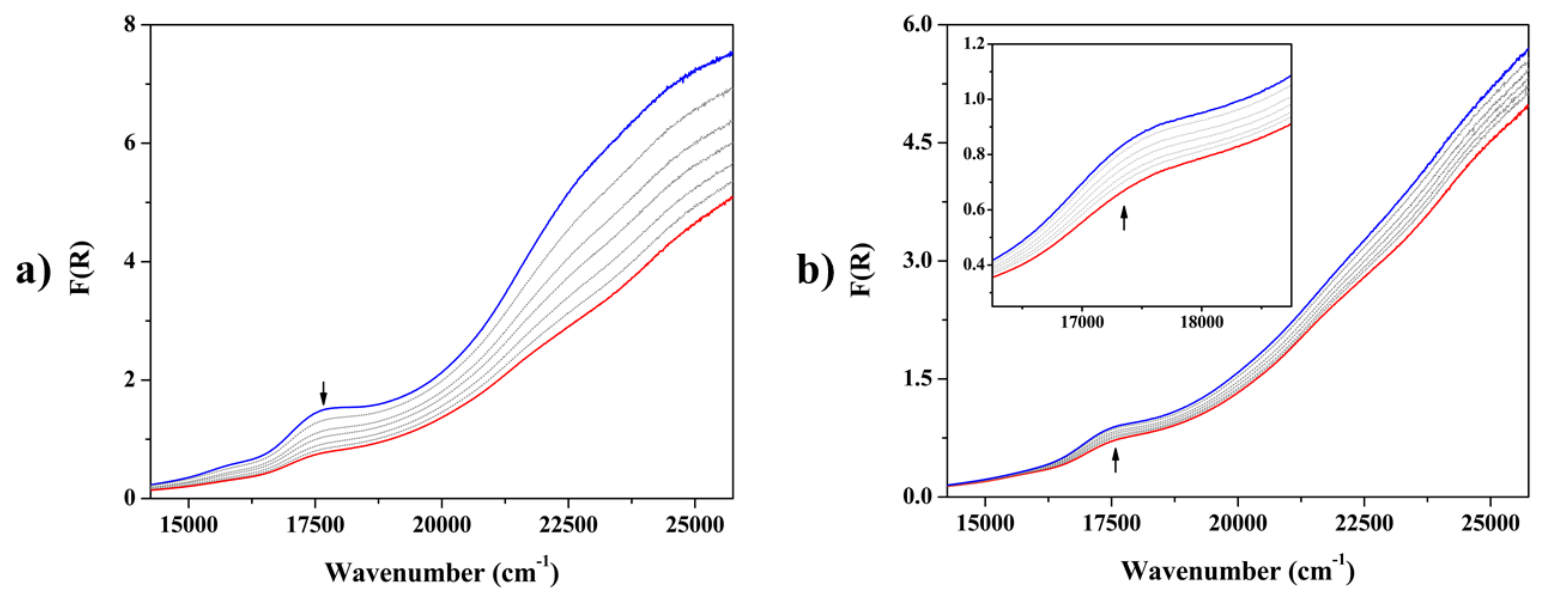

Fig. S51. Solid-state UV-vis spectra from $14250-25750 \mathrm{~cm}^{-1}$ for DUT-5(indoline) 2.5 (DASA), where the MOF was irradiated with white light up to 120 minutes (a) and heated to $100^{\circ} \mathrm{C}(\mathrm{b})$.

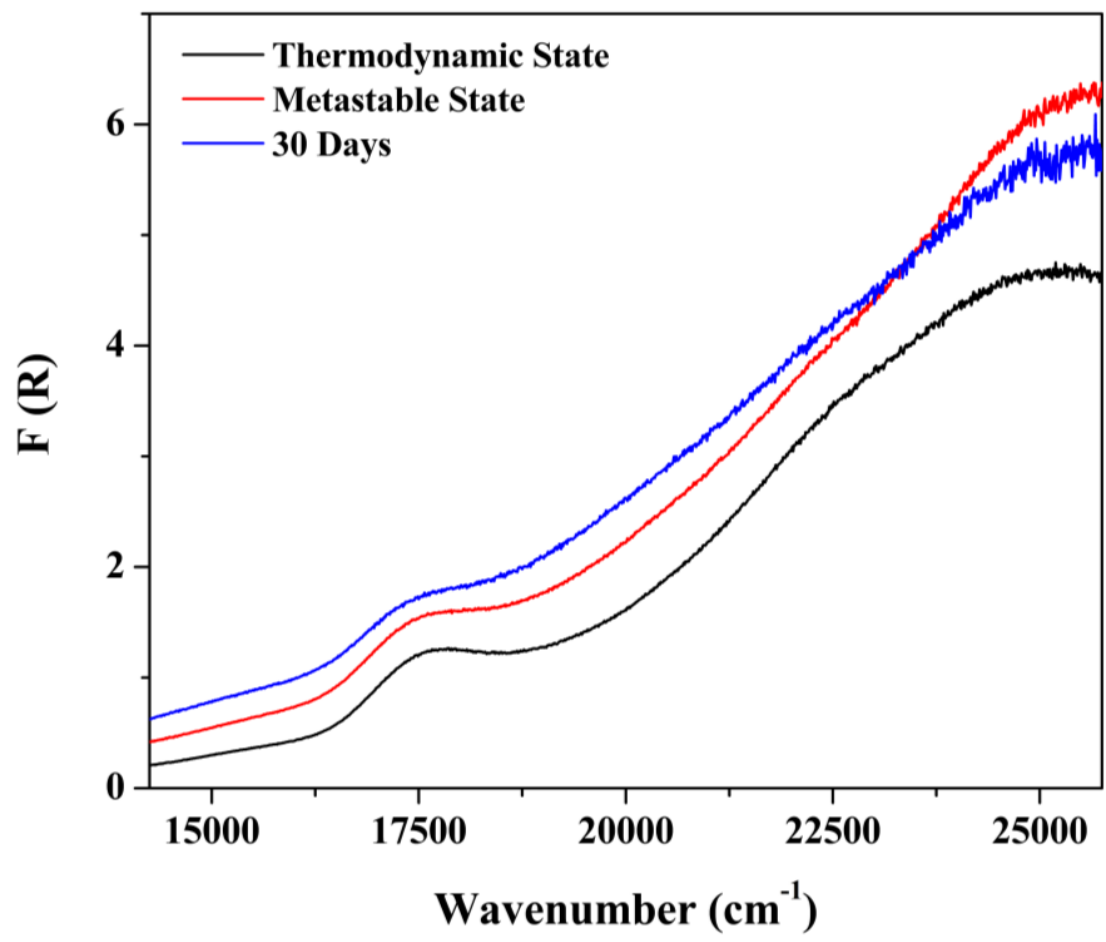

Fig. S52. Solid-state UV-vis spectra from $14250-25750 \mathrm{~cm}^{-1}$ for DUT-5(indoline) 0.5 (DASA), 30 days after irradiation with white light. 


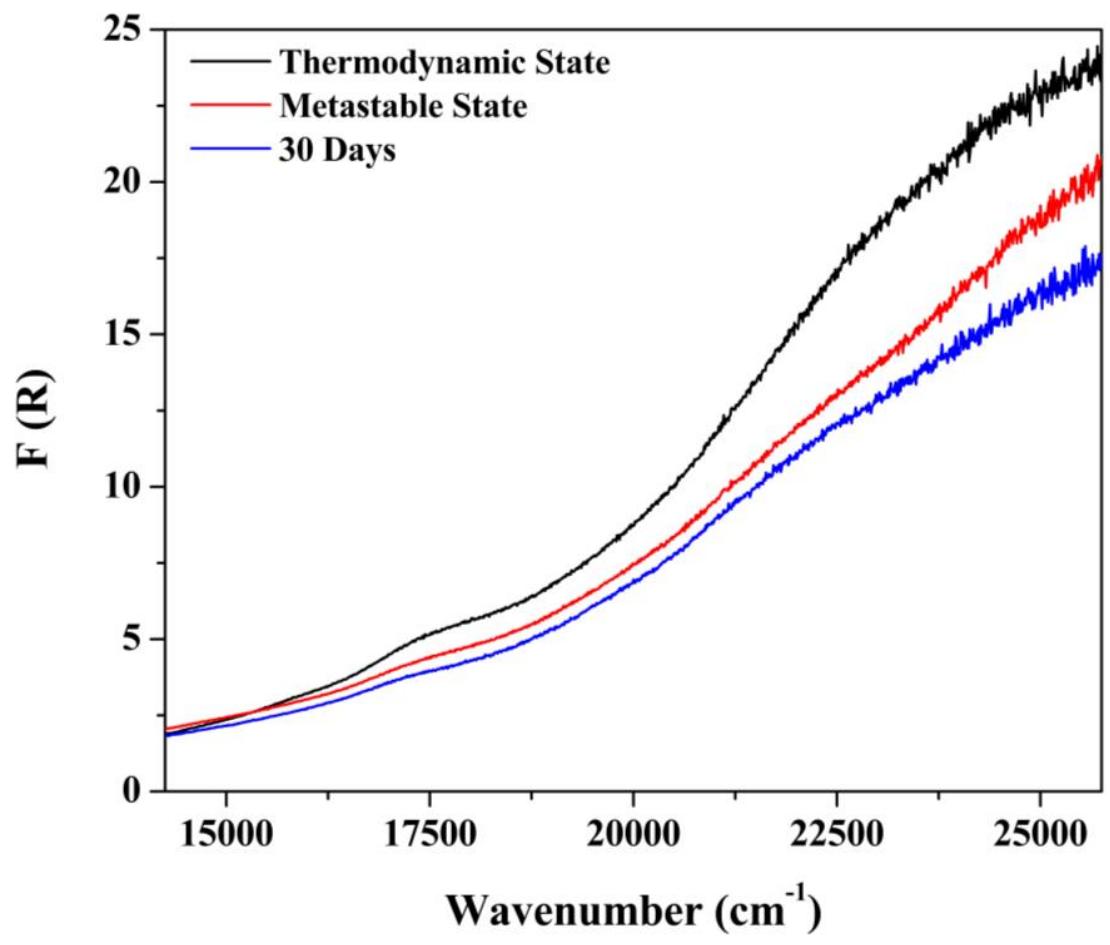

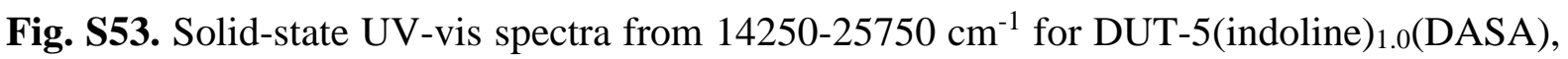
30 days after irradiation with white light.

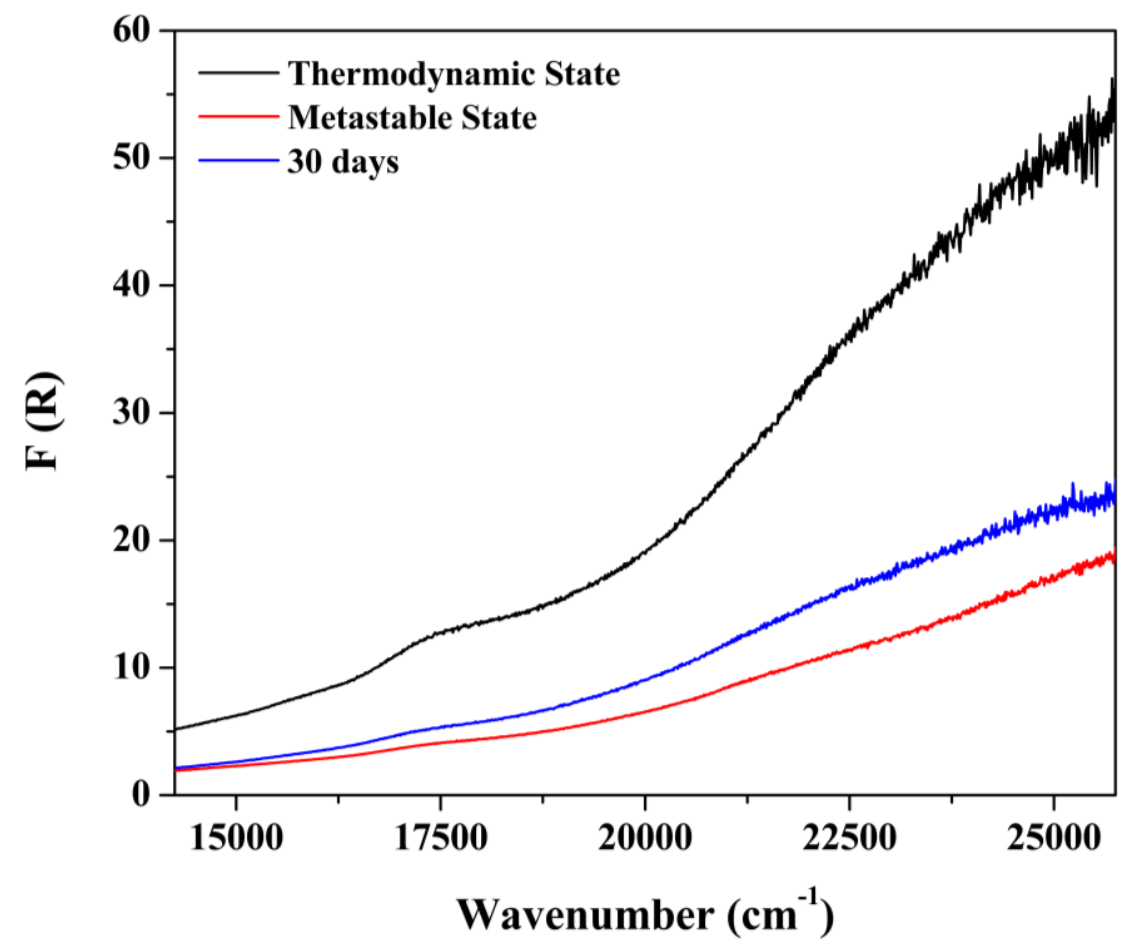

Fig. S54. Solid-state UV-vis spectra from $14250-25750 \mathrm{~cm}^{-1}$ for DUT-5(indoline) ${ }_{1.5}$ (DASA), 30 days after irradiation with white light. 


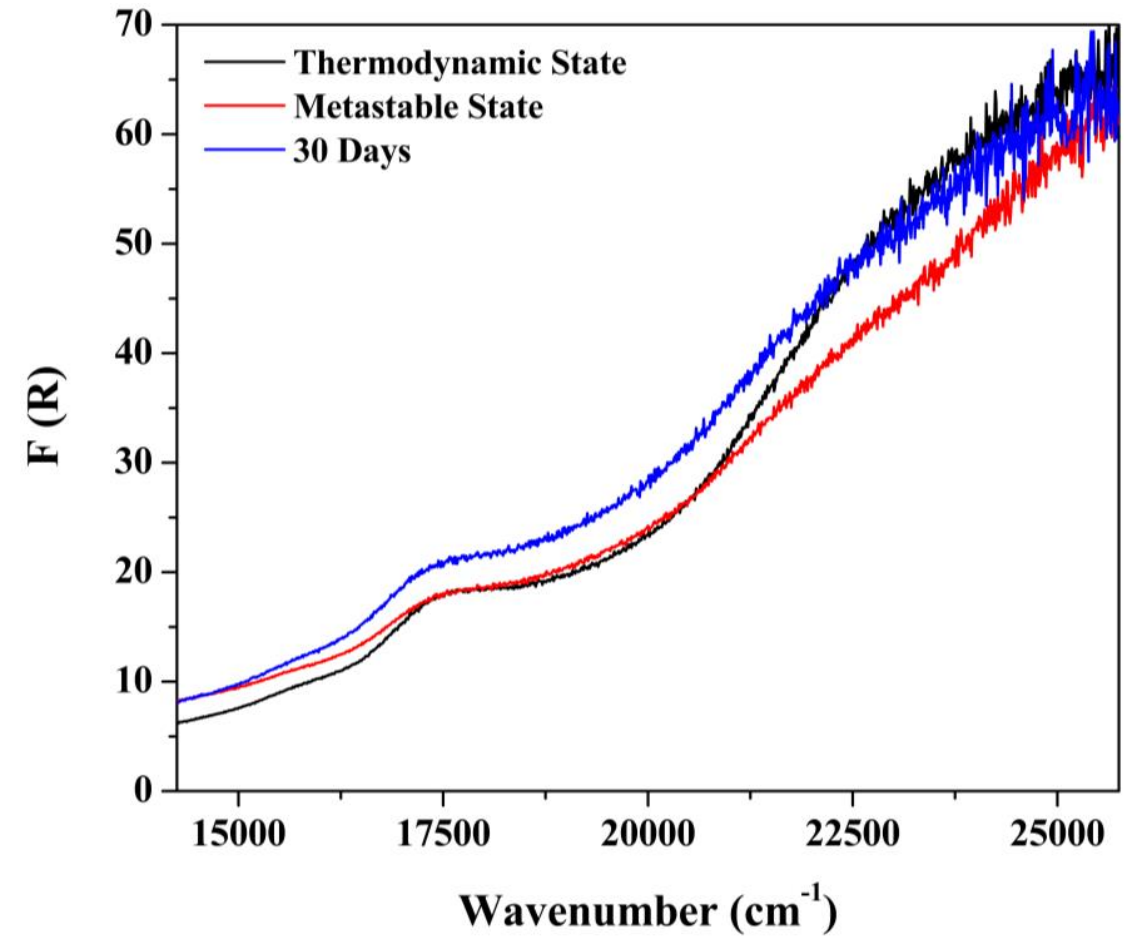

Fig. S55. Solid-state UV-vis spectra from $14250-25750 \mathrm{~cm}^{-1}$ for DUT-5(indoline) 2.0(DASA), 30 days after irradiation with white light.

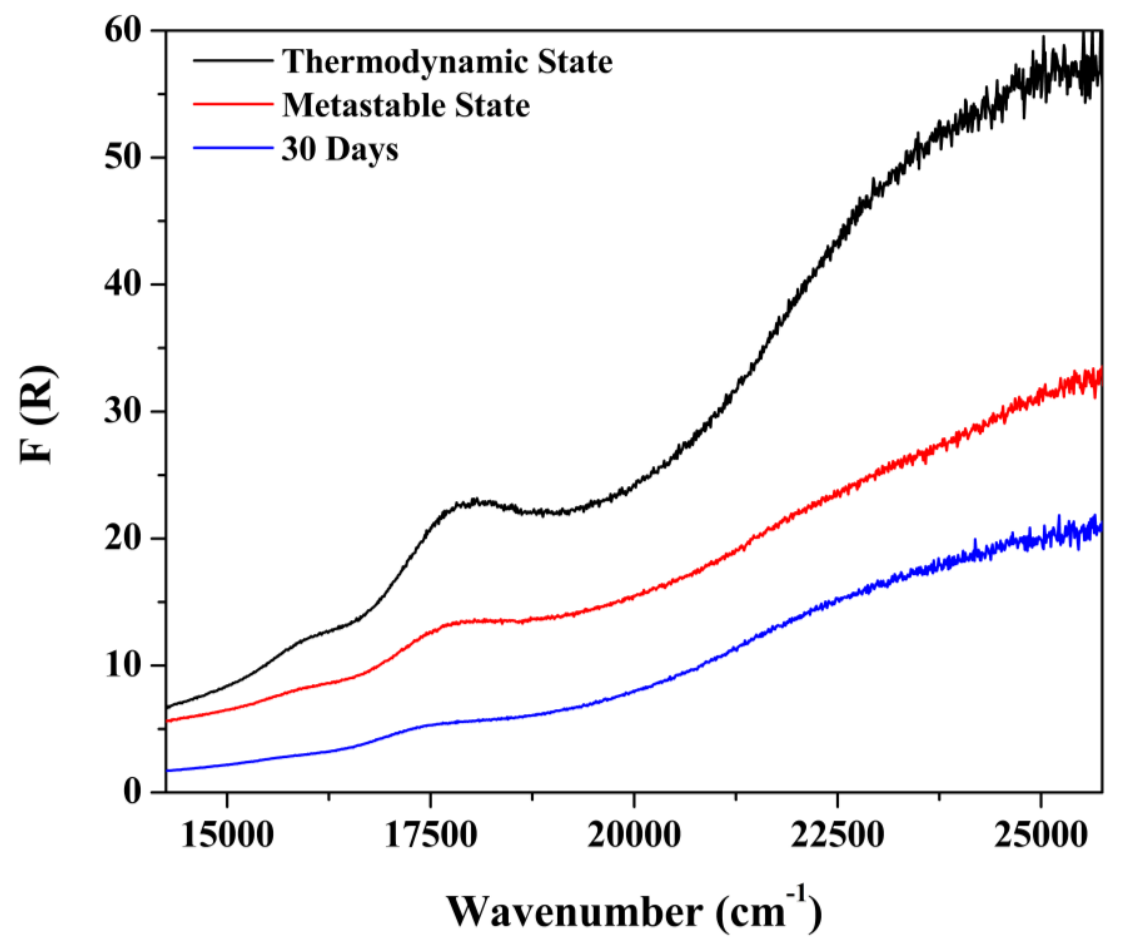

Fig. S56. Solid-state UV-vis spectra from $14250-25750 \mathrm{~cm}^{-1}$ for DUT-5(indoline) 2.5 (DASA), 30 days after irradiation with white light. 


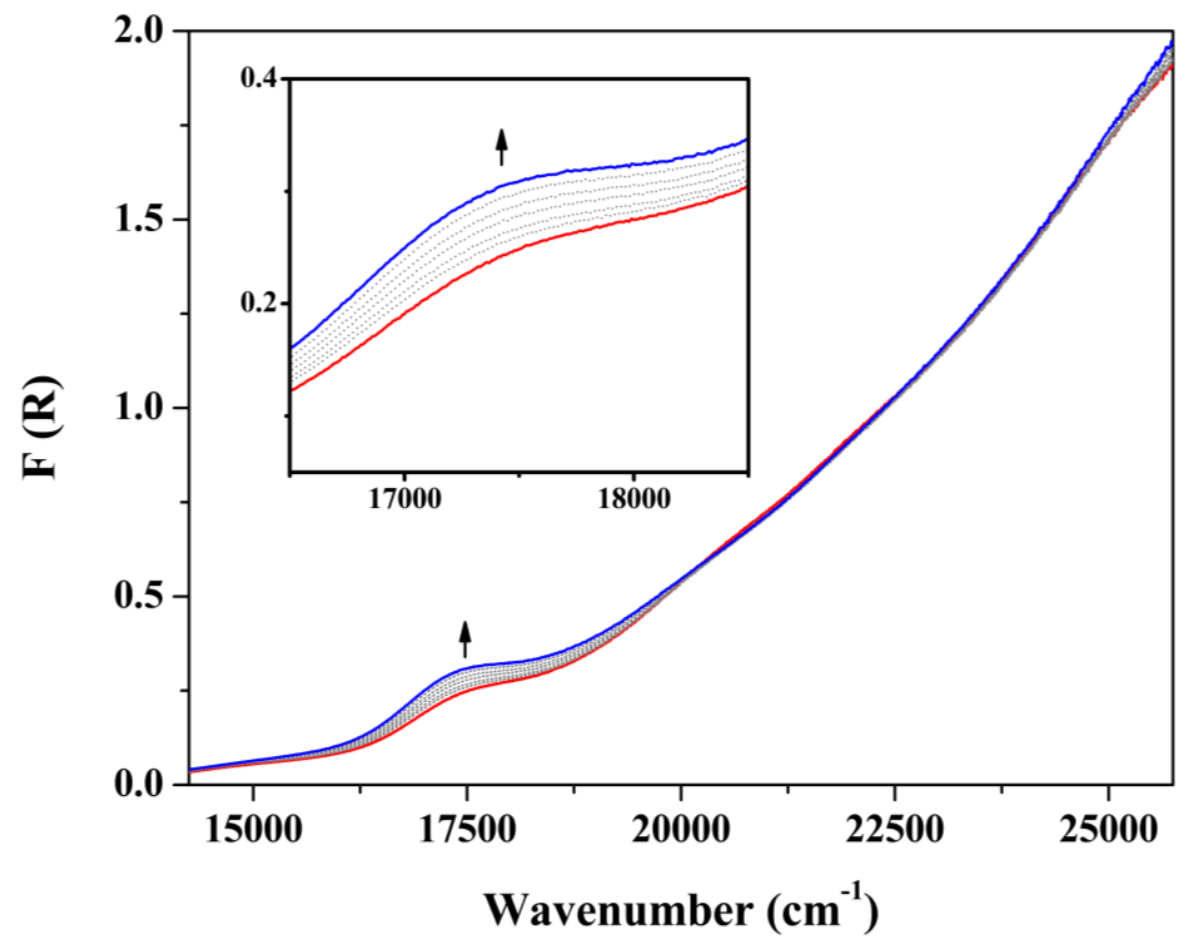

Fig. S57. Solid-state UV-vis spectra from $14250-25750 \mathrm{~cm}^{-1}$ for DUT-5(indoline) 0.5 (DASA), where heating regenerated the initial thermodynamic state after 30 days.

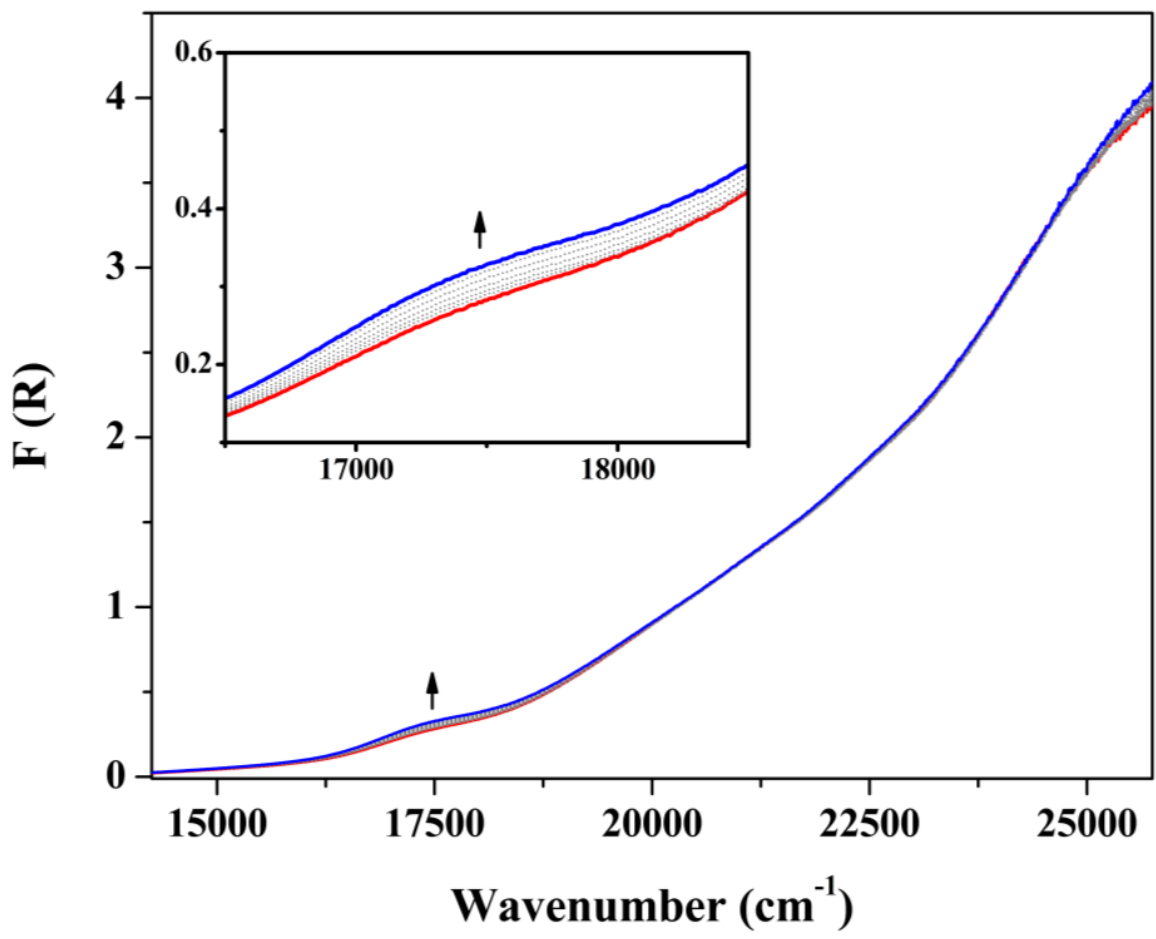

Fig. S58. Solid-state UV-vis spectra from $14250-25750 \mathrm{~cm}^{-1}$ for DUT-5(indoline) 1.0 (DASA), where heating regenerated the initial thermodynamic state after 30 days. 


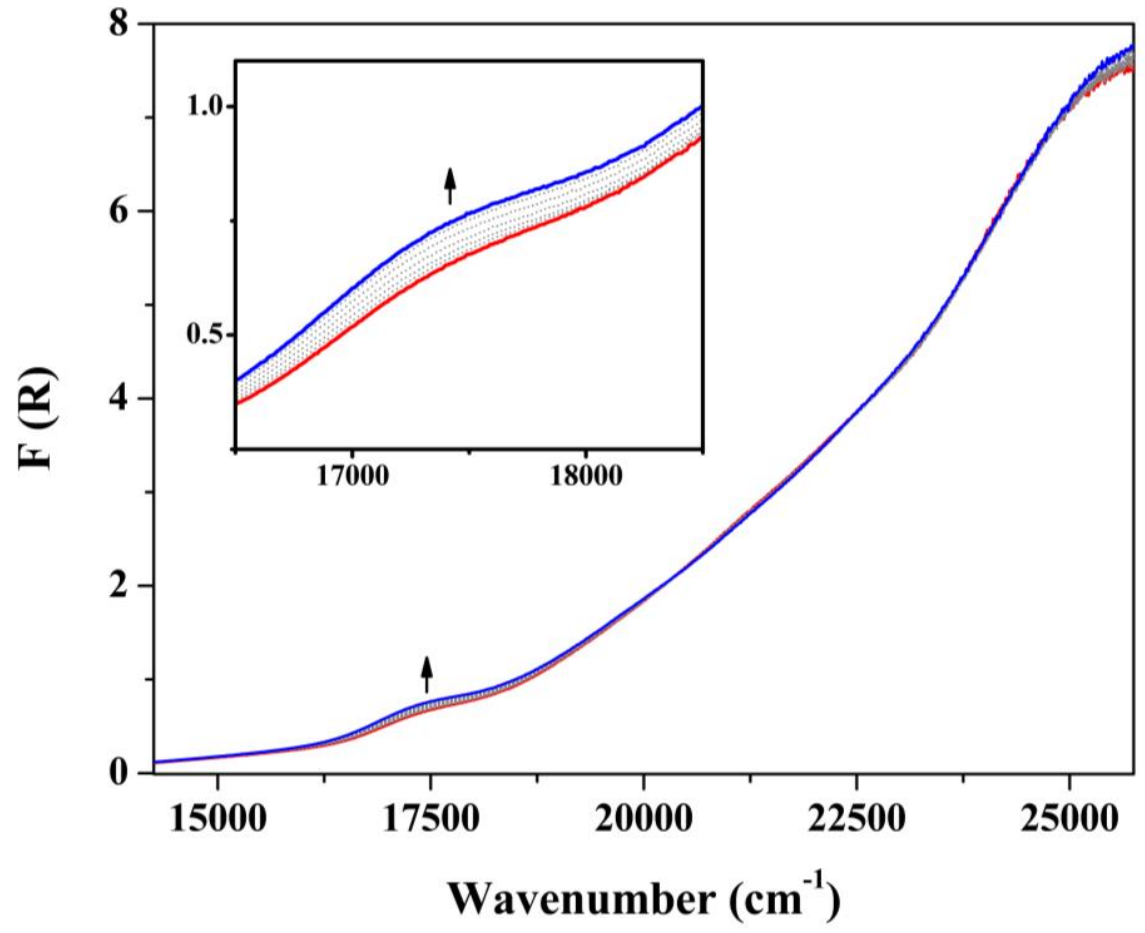

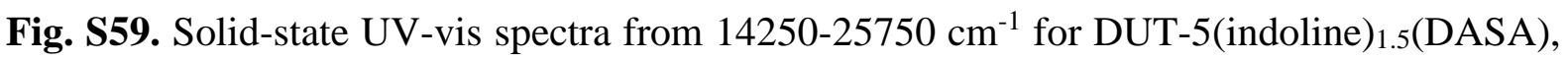
where heating regenerated the initial thermodynamic state after 30 days.

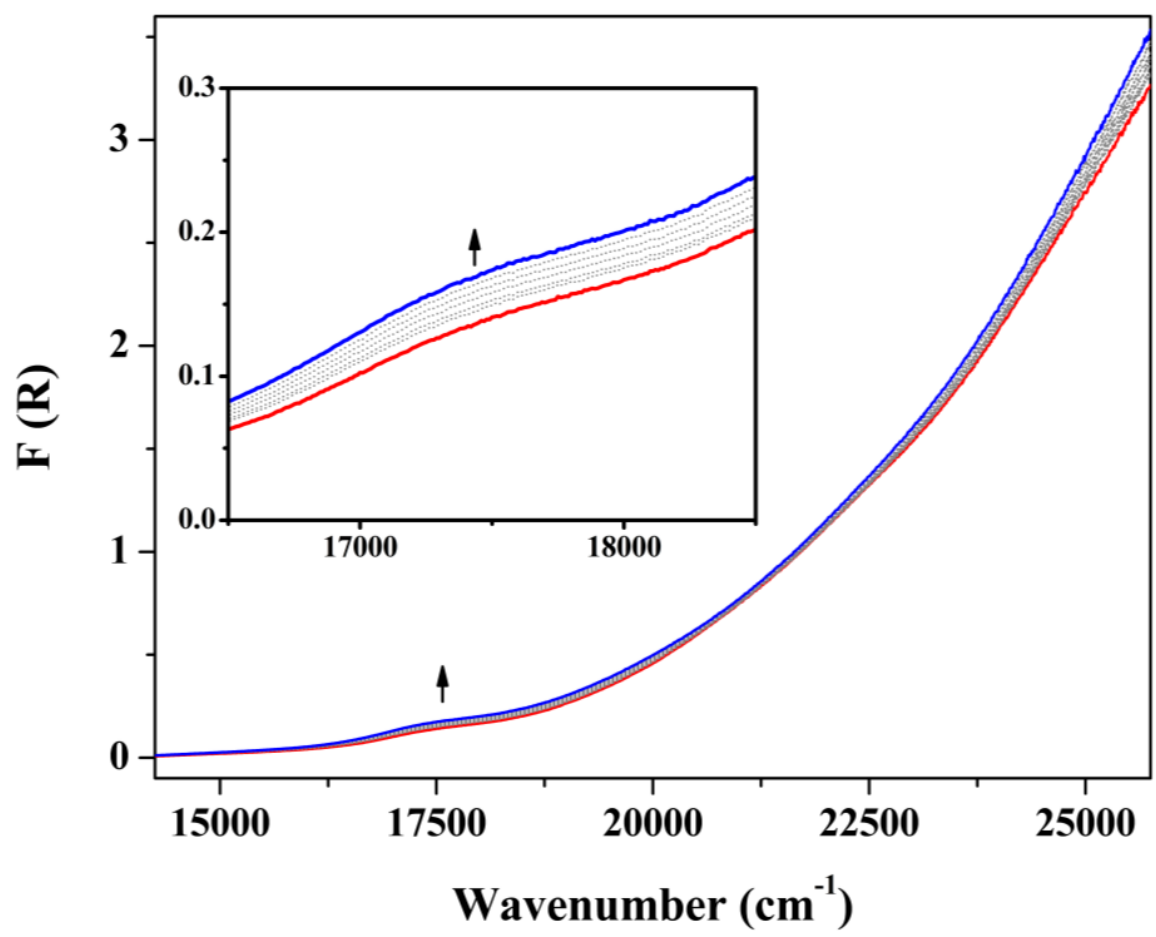

Fig. S60. Solid-state UV-vis spectra from $14250-25750 \mathrm{~cm}^{-1}$ for DUT-5(indoline) $)_{2.0}($ DASA), where heating regenerated the initial thermodynamic state after 30 days. 


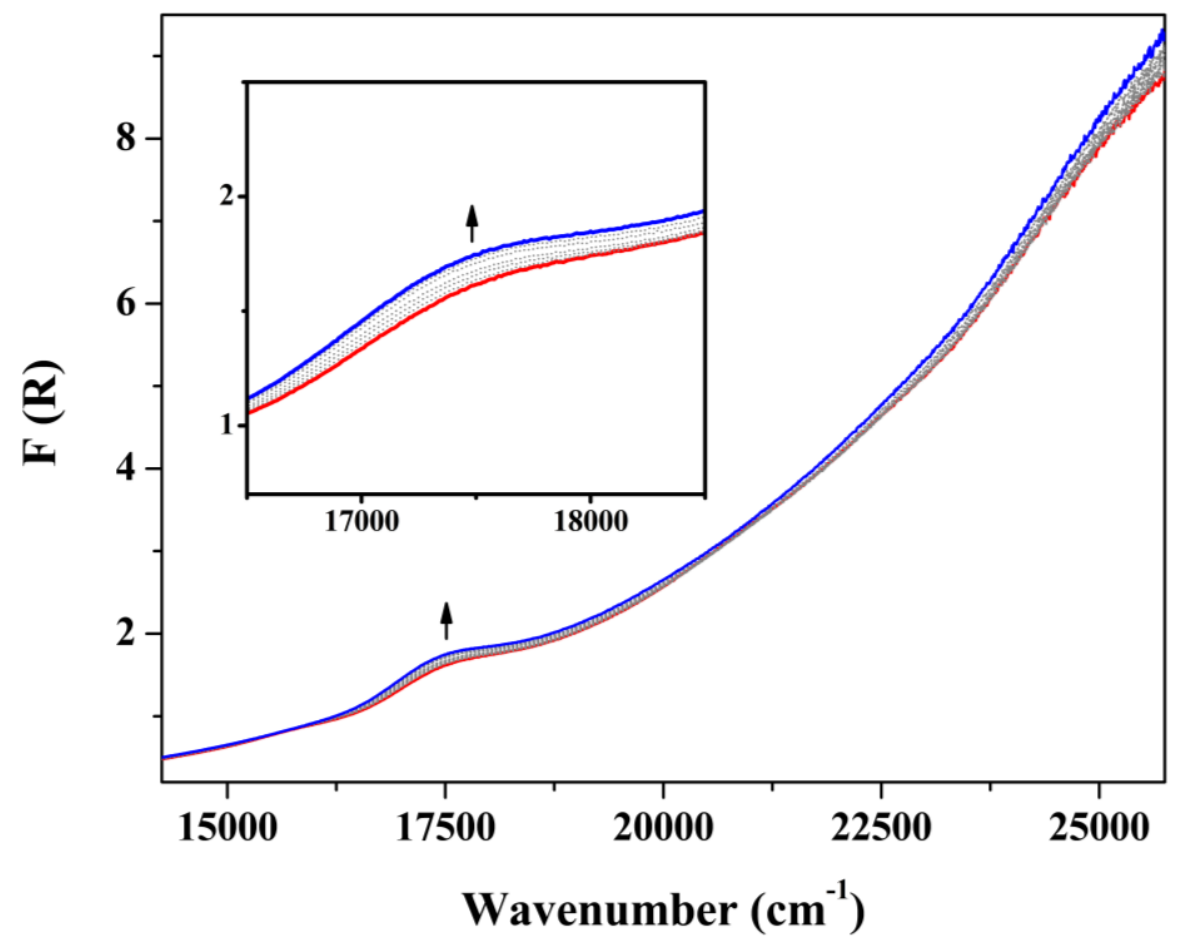

Fig. S61. Solid-state UV-vis spectra from $14250-25750 \mathrm{~cm}^{-1}$ for DUT-5(indoline) 2.5 (DASA), where heating regenerated the initial thermodynamic state after 30 days.

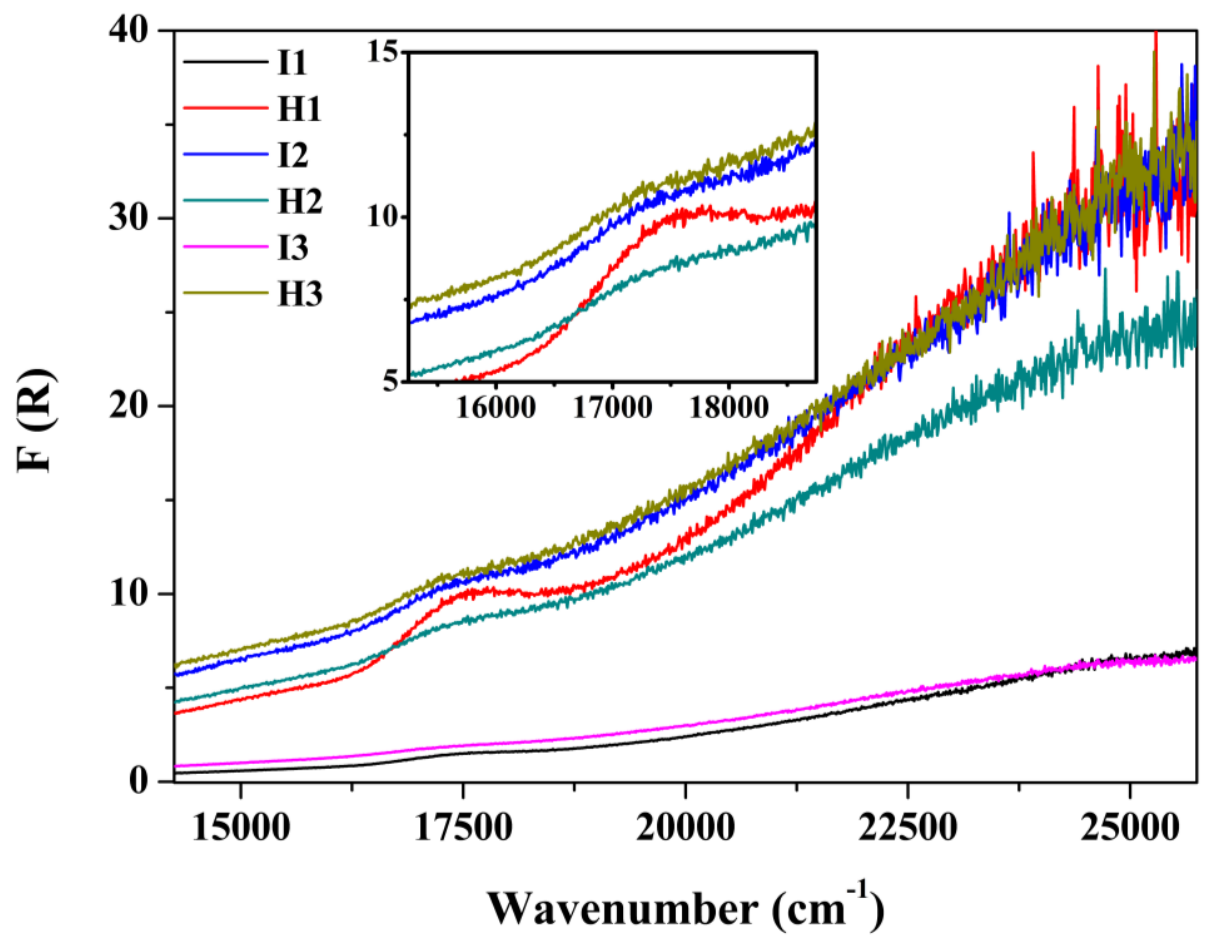

Fig. S62. Solid-state UV-vis spectra from $14250-25750 \mathrm{~cm}^{-1}$ for DUT-5(indoline) ${ }_{0.5}$ (DASA), over three cycles. 


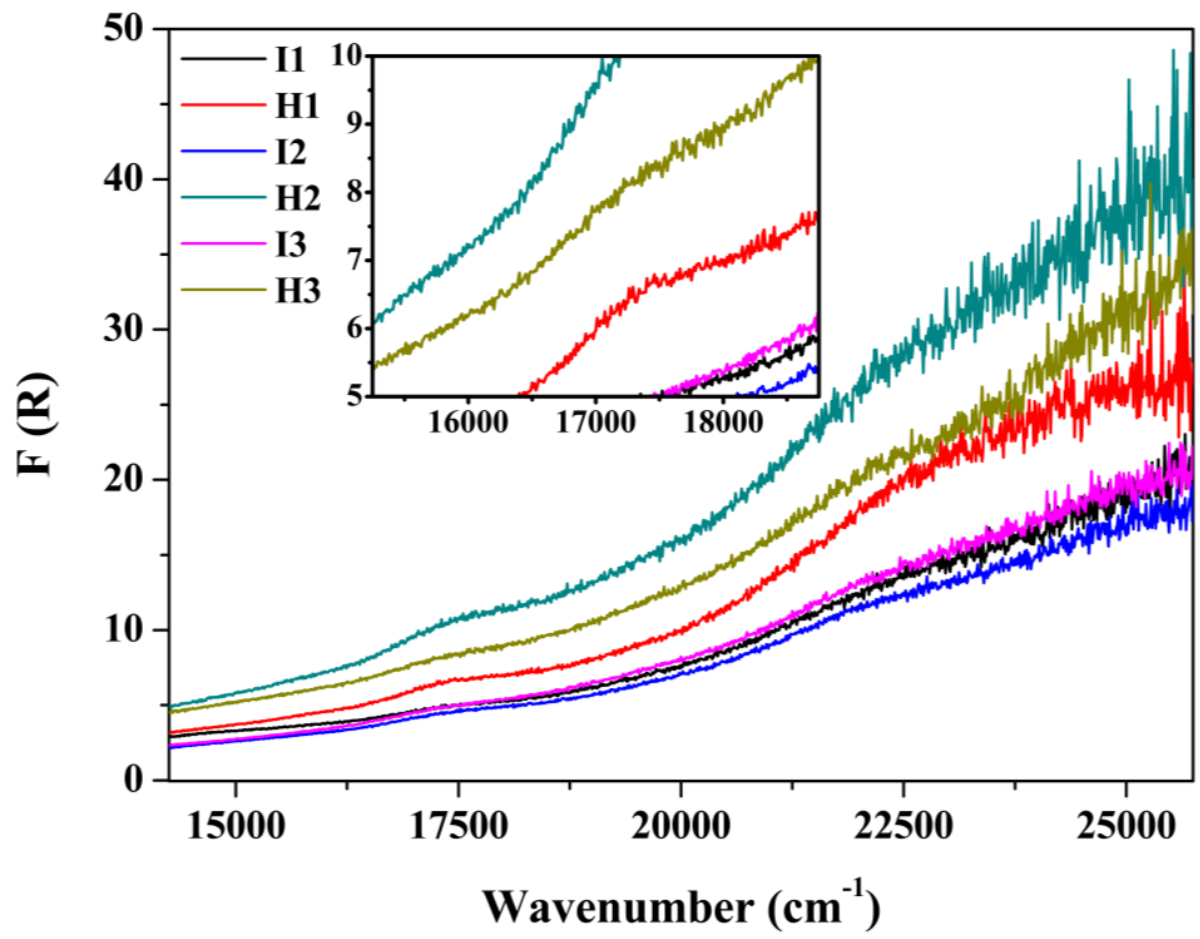

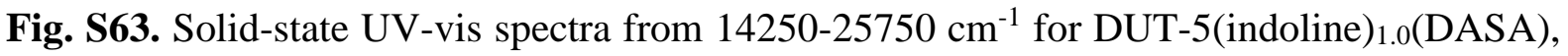
over three cycles.

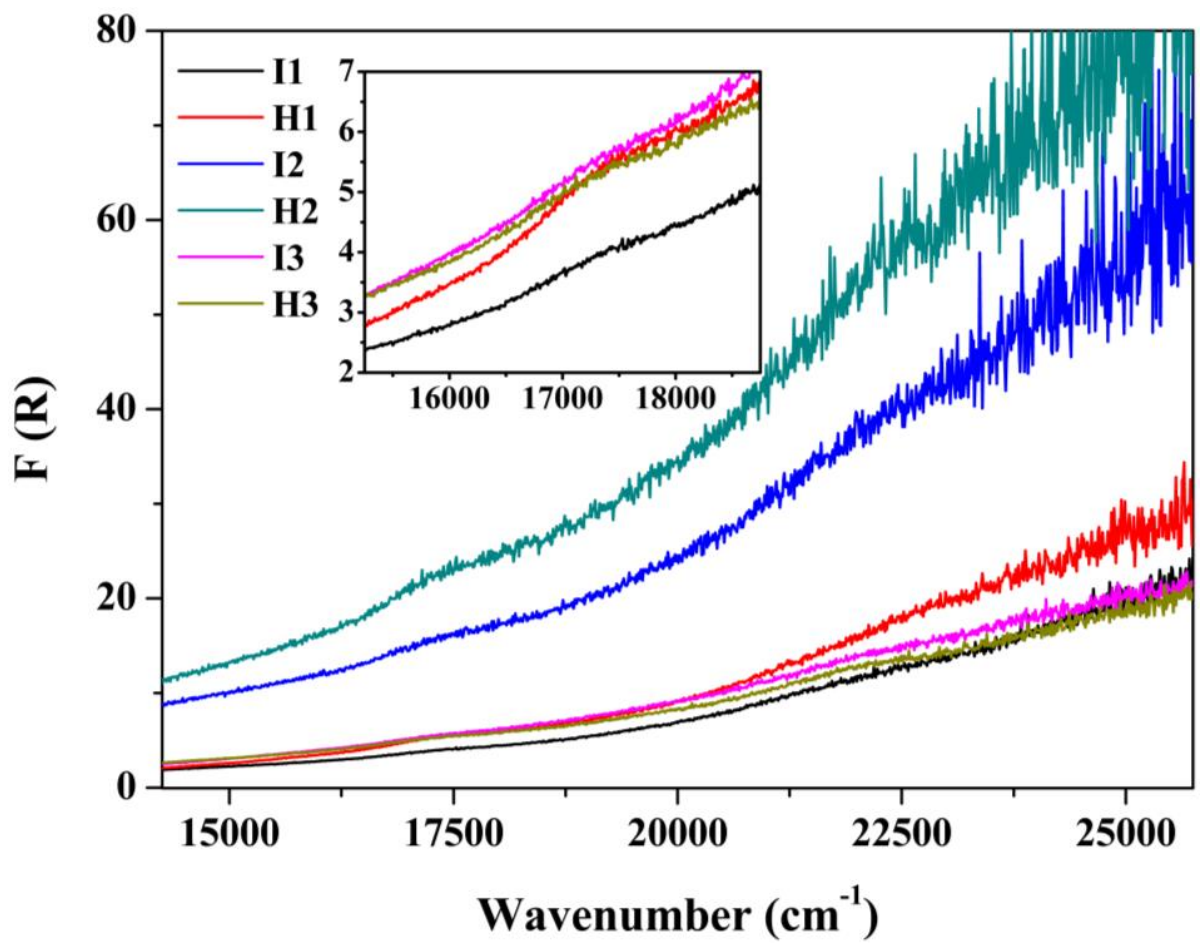

Fig. S64. Solid-state UV-vis spectra from $14250-25750 \mathrm{~cm}^{-1}$ for DUT-5(indoline) $)_{1.5}$ (DASA), over three cycles. 


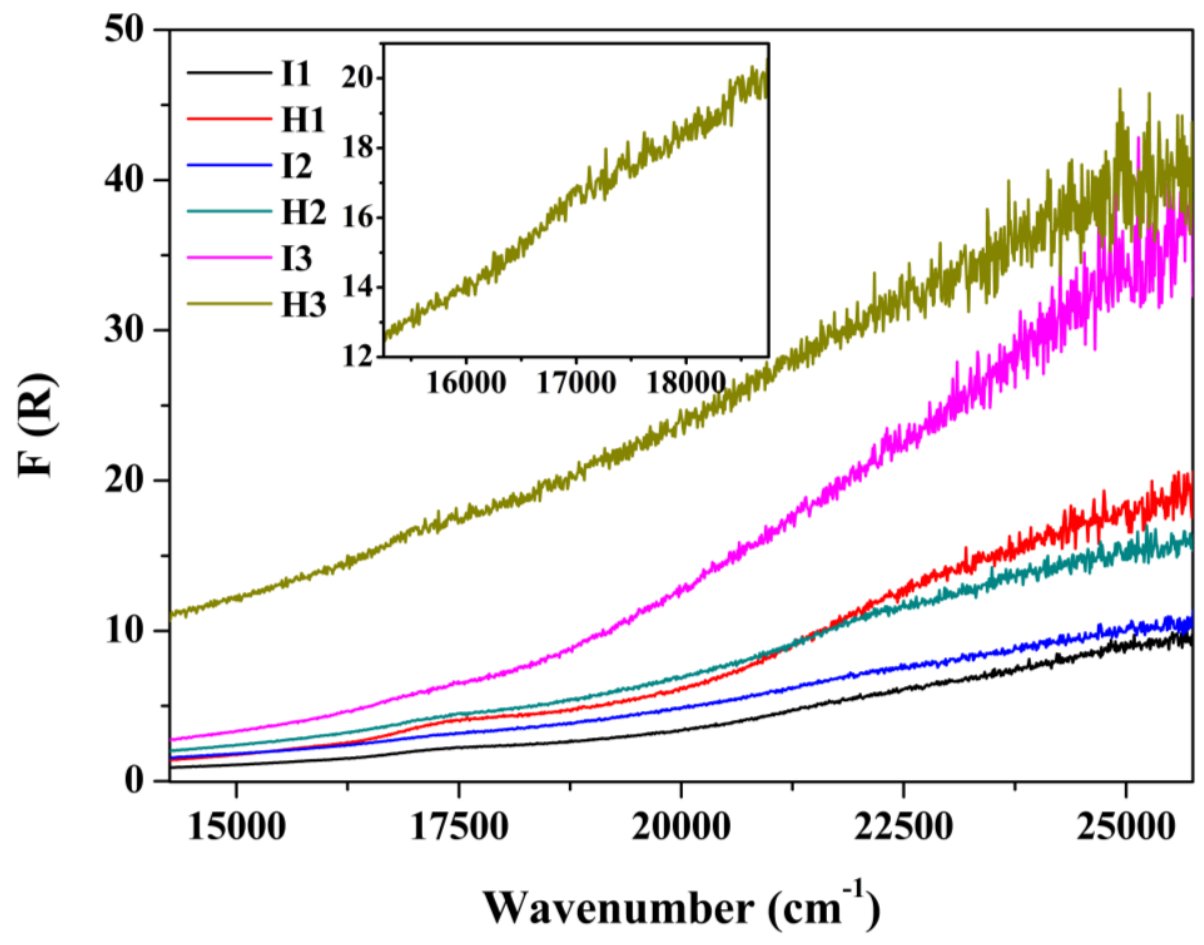

Fig. S65. Solid-state UV-vis spectra from $14250-25750 \mathrm{~cm}^{-1}$ for DUT-5(indoline) 2.0 (DASA), over three cycles.

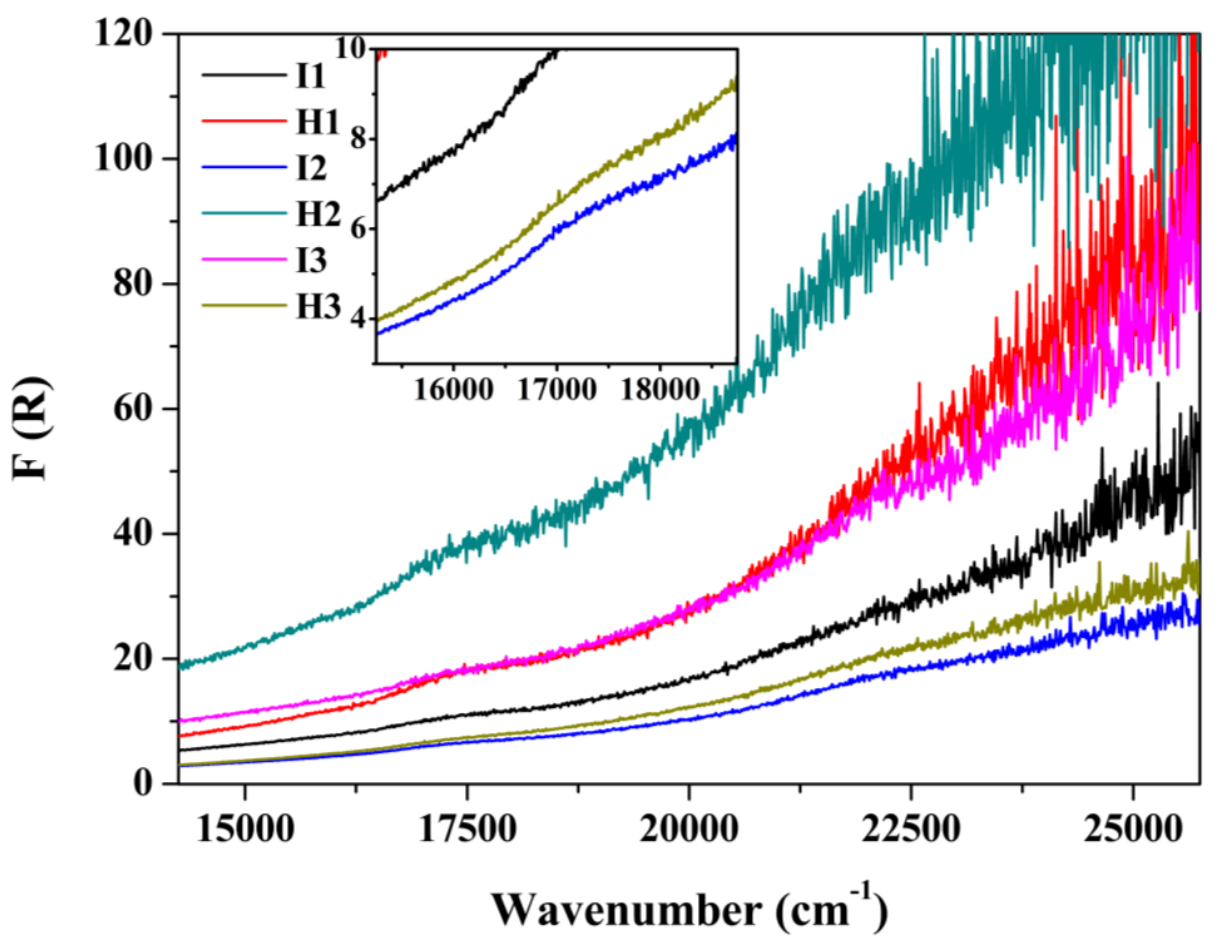

Fig. S66. Solid-state UV-vis spectra from $14250-25750 \mathrm{~cm}^{-1}$ for DUT-5(indoline) $)_{2.5}(\mathrm{DASA})$, over three cycles. 


\section{S7. Thermogravimetric Analysis (TGA)}

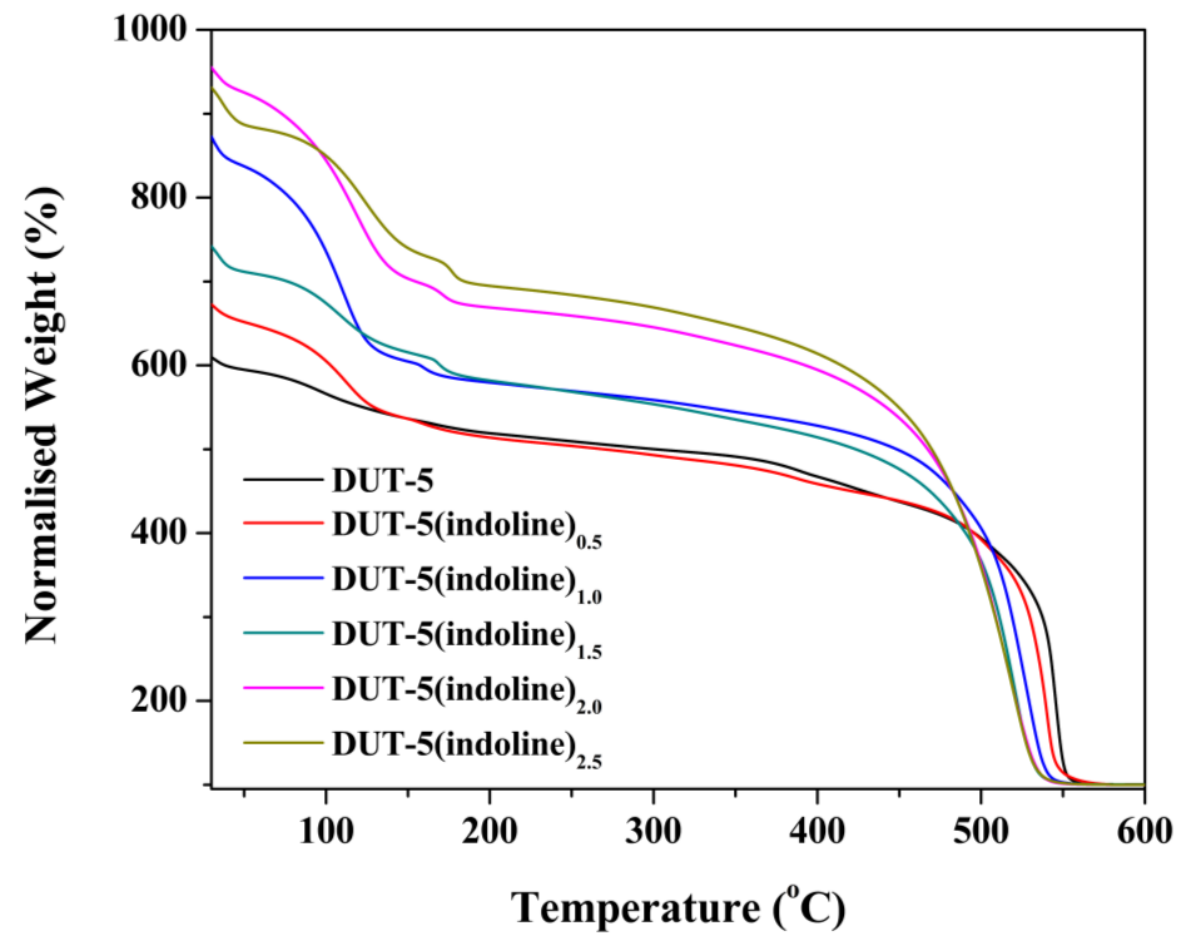

Fig. S67. Normalized TGA for DUT-5 and DUT-5(indoline)0.5-2.5. Normalization was conducted assuming $100 \% \mathrm{Al}_{2} \mathrm{O}_{3}$ yield.

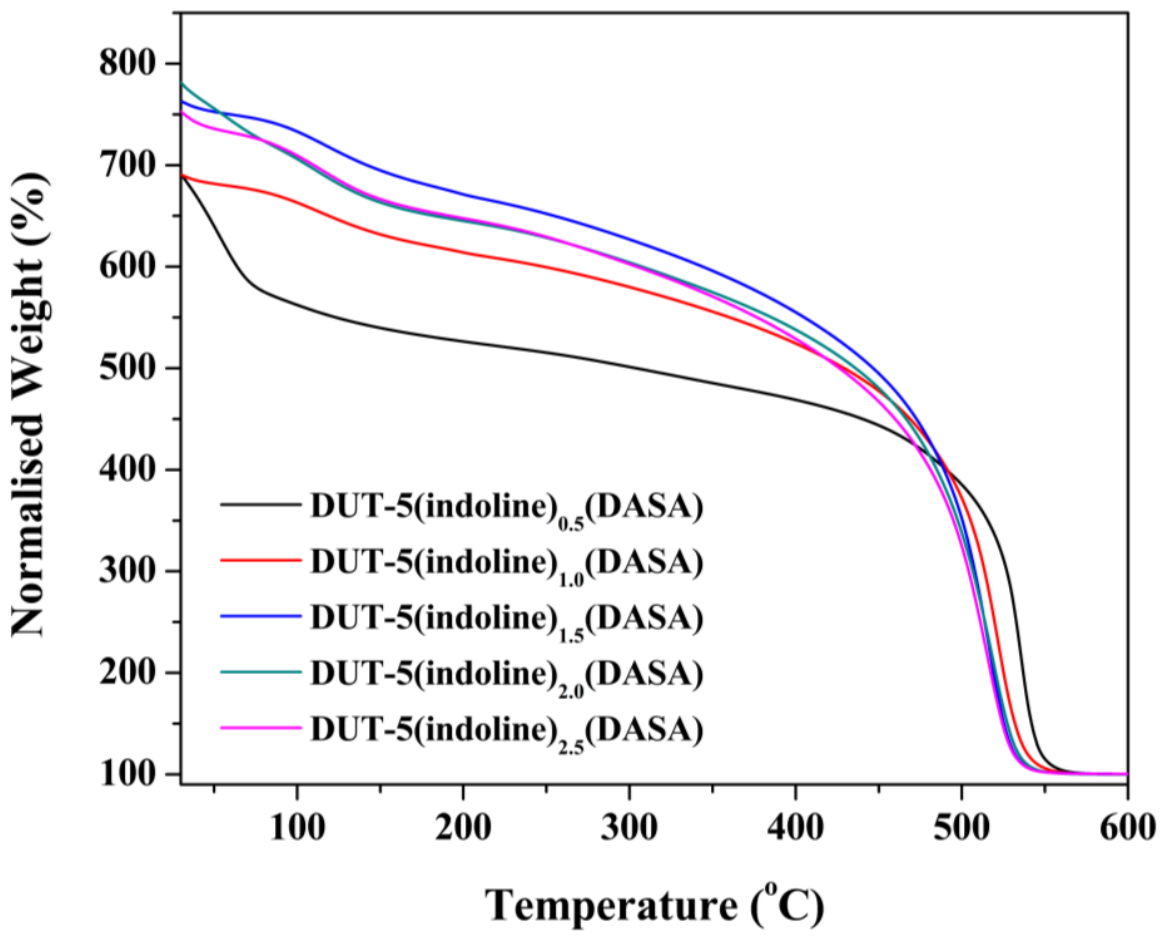

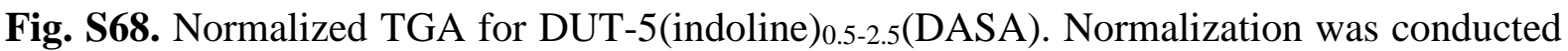
assuming $100 \% \mathrm{Al}_{2} \mathrm{O}_{3}$ yield. 
TGA results (Fig. S67 and S68) were inconsistent with trends commonly associated with defect series in the literature. ${ }^{8-11}$ For DUT-5(indoline) $)_{0.5-2.5}$ an increase in organic matter with respect to $\mathrm{Al}_{2} \mathrm{O}_{3}$ yield was observed. This was proposed to be the result of the molecular mass of the indoline modulator which is considerably greater than that of other species exploited in a similar manner for defect generation. ${ }^{8-11}$ PSM of the defect series to generate DUT5 (indoline) $)_{0.5-2.5}$ (DASA) resulted in an increase in the organic content by comparison to the relevant precursor framework. In addition, the same overall trend of increasing organic matter from least to most defective MOFs was also observed for the DUT-5(indoline) $0.5-2.5$ (DASA). 


\section{S8. Molecular Formulae Determination}

Table S4. Molecular formulae for the DUT-5(indoline) 0.5-2.5 series (data used for calculation included).

\begin{tabular}{cccc}
\hline Framework & bpdc $^{2-}: \mathrm{C}_{12} \mathrm{H}_{12} \mathrm{NO}_{2}$ & TGA Plateau $\left(450^{\circ} \mathrm{C}\right)$ & Formula \\
\hline DUT-5(indoline) $)_{0.5}$ & $1.29: 1.00$ & 49.63 & {$\left[\mathrm{Al}(\mathrm{bpdc})_{0.51}\left(\mathrm{C}_{12} \mathrm{H}_{12} \mathrm{NO}_{2}\right)_{0.40}\right]^{+1.58}$} \\
DUT-5(indoline) $)_{1.0}$ & $2.22: 1.00$ & 55.01 & {$\left[\mathrm{Al}(\mathrm{bpdc})_{0.60}\left(\mathrm{C}_{12} \mathrm{H}_{12} \mathrm{NO}_{2}\right)_{0.27}\right]^{+1.53}$} \\
DUT-5(indoline) $)_{1.5}$ & $3.14: 1.00$ & 60.78 & {$\left[\mathrm{Al}(\mathrm{bpdc})_{0.69}\left(\mathrm{C}_{12} \mathrm{H}_{12} \mathrm{NO}_{2}\right)_{0.22}^{+1.40}\right.$} \\
DUT-5(indoline) $)_{2.0}$ & $5.03: 1.00$ & 69.52 & {$\left[\mathrm{Al}(\mathrm{bpdc})_{0.77}\left(\mathrm{C}_{12} \mathrm{H}_{12} \mathrm{NO}_{2}\right)_{0.15}\right]^{+1.31}$} \\
DUT-5(indoline) $)_{2.5}$ & $7.03: 1.00$ & 64.84 & {$\left[\mathrm{Al}(\mathrm{bpdc})_{0.78}\left(\mathrm{C}_{12} \mathrm{H}_{12} \mathrm{NO}_{2}\right)_{0.11}^{+1.33}\right.$} \\
\hline
\end{tabular}

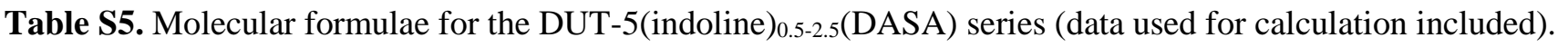

\begin{tabular}{cccc}
\hline Framework & bpdc $^{2-}: \mathrm{C}_{12} \mathrm{H}_{12} \mathrm{NO}_{2}: \mathrm{C}_{23} \mathrm{H}_{22} \mathrm{~N}_{3} \mathrm{O}_{6}$ & TGA Plateau $\left(450^{\circ} \mathrm{C}\right)$ & Formula \\
\hline DUT-5(indoline) $)_{0.5}($ DASA $)$ & $1.67: 1.00: 1.23$ & 62.90 & {$\left[\mathrm{Al}(\mathrm{bpdc})_{0.26}\left(\mathrm{C}_{12} \mathrm{H}_{12} \mathrm{NO}_{2}\right)_{0.15}\left(\mathrm{C}_{23} \mathrm{H}_{22} \mathrm{~N}_{3} \mathrm{O}_{6}\right)_{0.19}\right]^{+2.14}$} \\
DUT-5(indoline) $)_{1.0}$ (DASA) & $1.67: 1.00: 1.04$ & 68.88 & {$\left[\mathrm{Al}(\mathrm{bpdc})_{0.30}\left(\mathrm{C}_{12} \mathrm{H}_{12} \mathrm{NO}_{2}\right)_{0.18}\left(\mathrm{C}_{23} \mathrm{H}_{22} \mathrm{~N}_{3} \mathrm{O}_{6}\right)_{0.19}\right]^{+2.03}$} \\
DUT-5(indoline) $)_{1.5}$ (DASA) & $1.08: 1.00: 1.71$ & 64.58 & {$\left[\mathrm{Al}(\mathrm{bpdc})_{0.18}\left(\mathrm{C}_{12} \mathrm{H}_{12} \mathrm{NO}_{2}\right)_{0.17}\left(\mathrm{C}_{23} \mathrm{H}_{22} \mathrm{~N}_{3} \mathrm{O}_{6}\right)_{0.28}\right]^{+2.19}$} \\
DUT-5(indoline) $)_{2.0}$ (DASA) & $1.59: 0.00: 1.00$ & 60.53 & {$\left[\mathrm{Al}(\mathrm{bpdc})_{0.38}\left(\mathrm{C}_{23} \mathrm{H}_{22} \mathrm{~N}_{3} \mathrm{O}_{6}\right)_{0.24}\right]^{+2.00}$} \\
DUT-5(indoline) $)_{2.5}$ (DASA) & $1.35: 0.00: 1.00$ & 61.67 & {$\left[\mathrm{Al}(\mathrm{bpdc})_{0.33}\left(\mathrm{C}_{23} \mathrm{H}_{22} \mathrm{~N}_{3} \mathrm{O}_{6}\right)_{0.25}\right]^{+2.09}$} \\
\hline
\end{tabular}


S9. $\mathbf{N}_{2}$ Physisorption (77 K)

a)
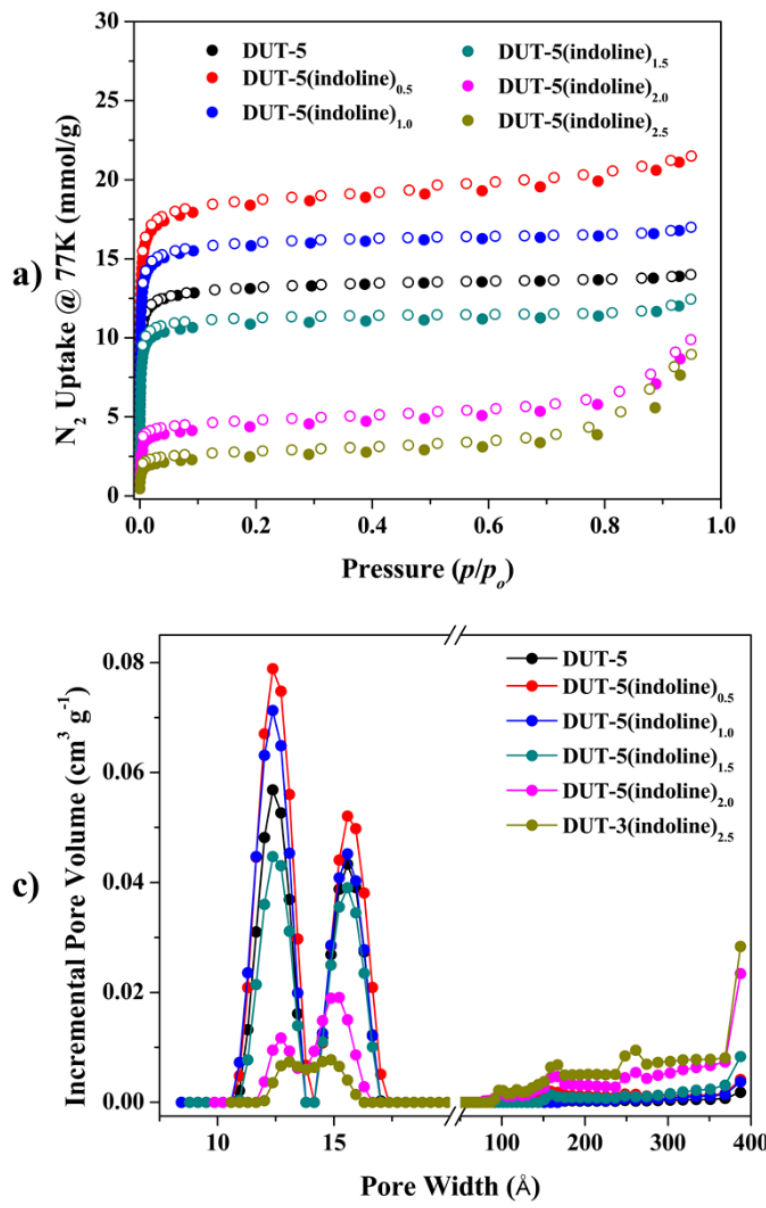
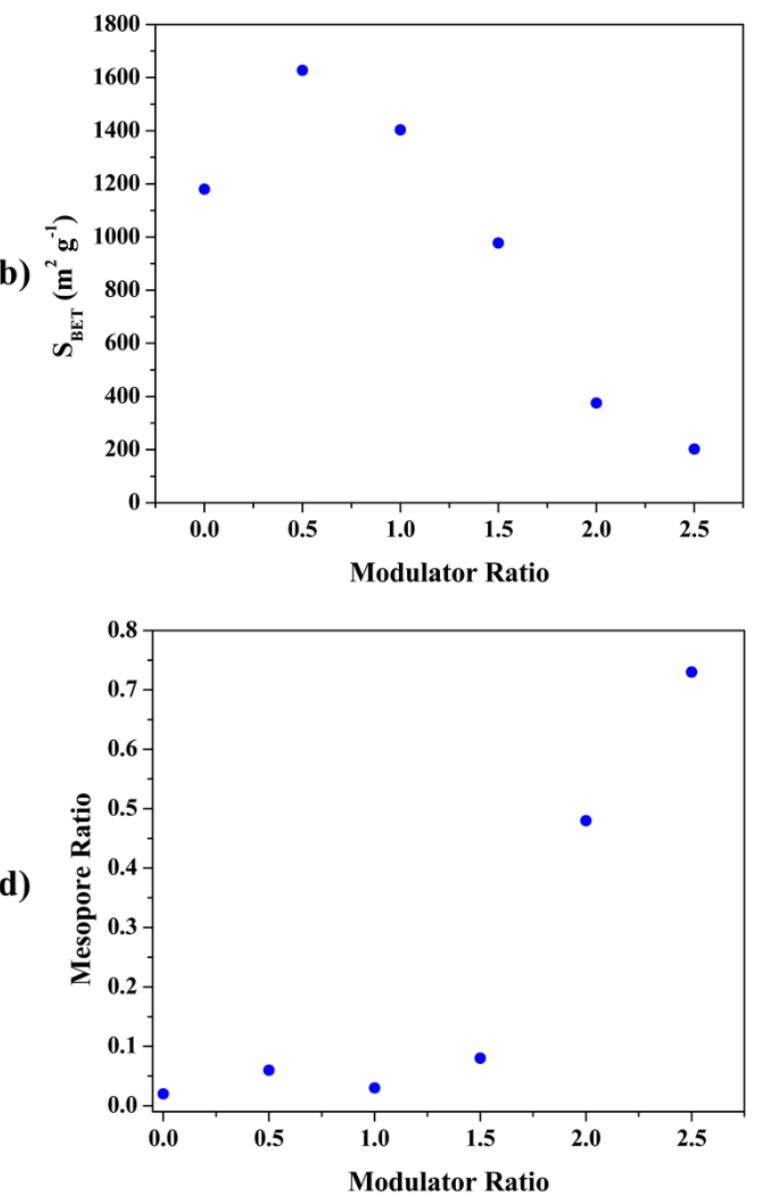

Fig. S69. $\mathrm{N}_{2}$ physisorption isotherms $(77 \mathrm{~K} \text { ) for DUT-5 and DUT-5(indoline) })_{0.5-2.5}$ (a) with $\mathrm{S}_{\mathrm{BET}}(\mathrm{b})$, NLDFT pore size distributions (c) and mesopore ratios (d) included. 

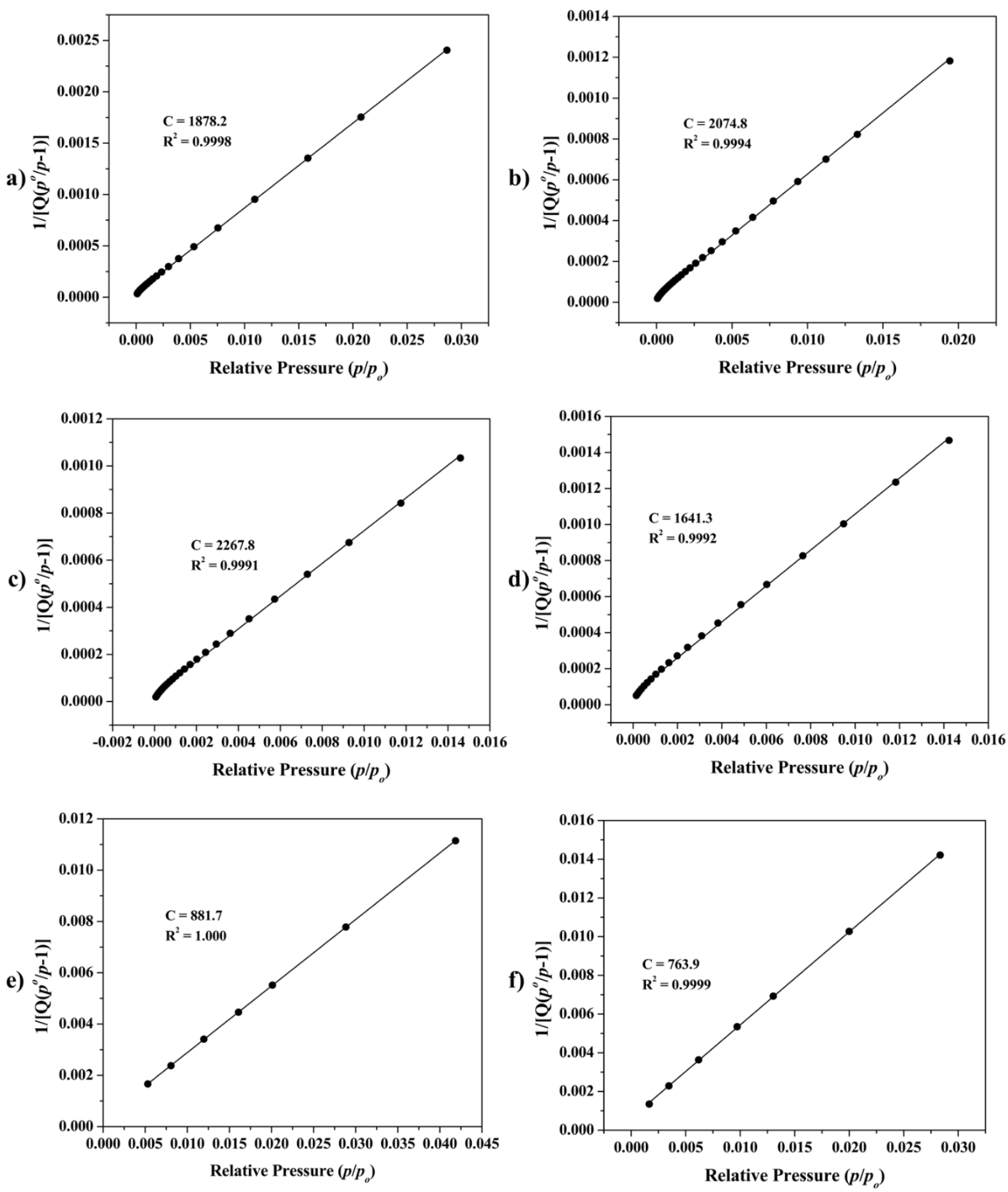

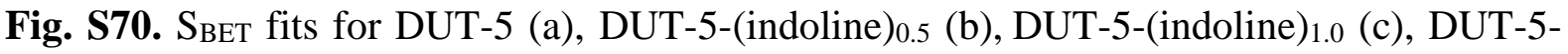
(indoline) $)_{1.5}$ (d), DUT-5-(indoline) 2.0 (e) and DUT-5-(indoline) 2.5 (f). 

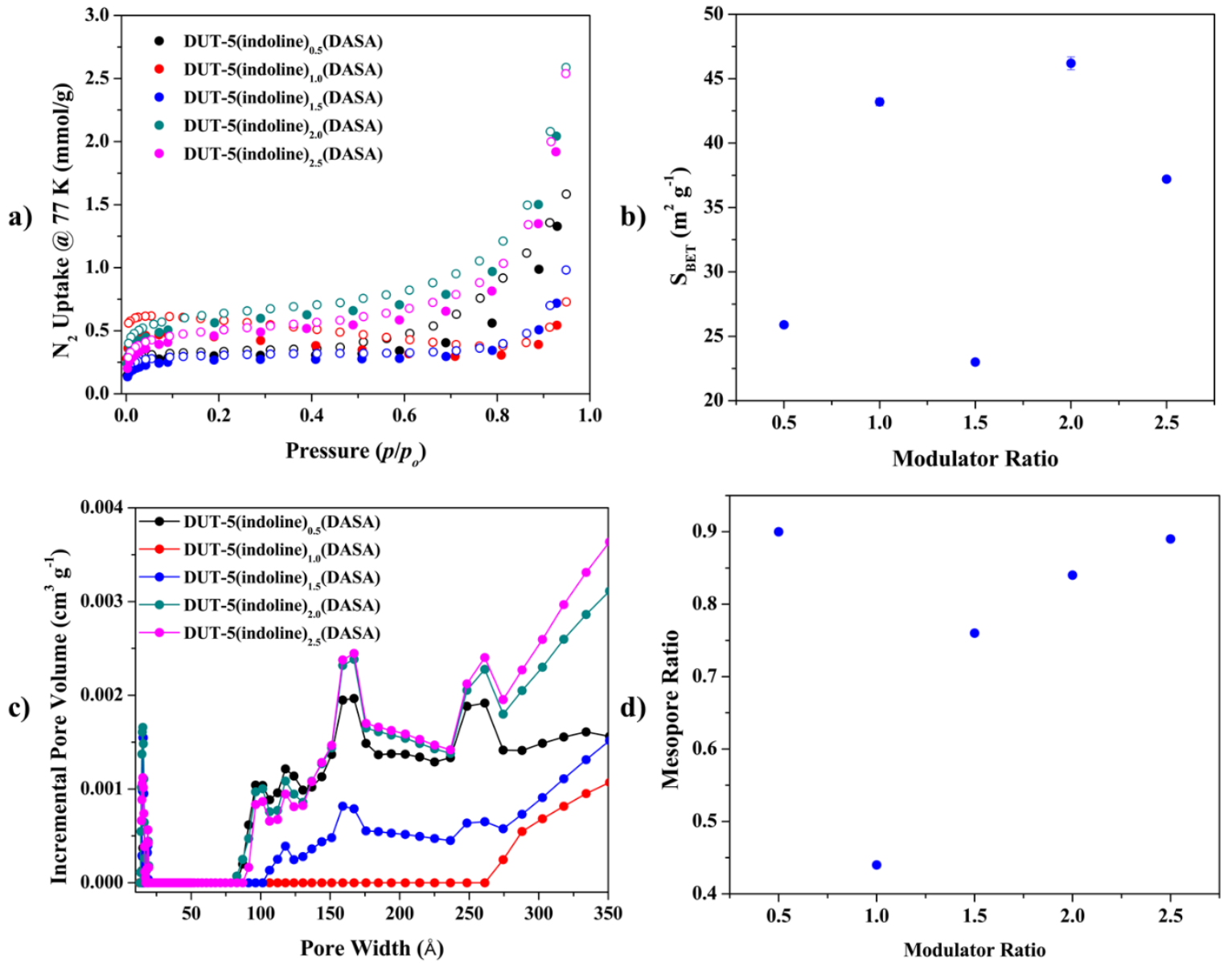

Fig. S71. $\mathrm{N}_{2}$ physisorption isotherms $\left(77 \mathrm{~K}\right.$ ) for DUT-5(indoline) 0.5-2.5 (DASA) (a) with $\mathrm{S}_{\mathrm{BET}}$ (b), NLDFT pore size distributions (c) and mesopore ratios (d) included. 

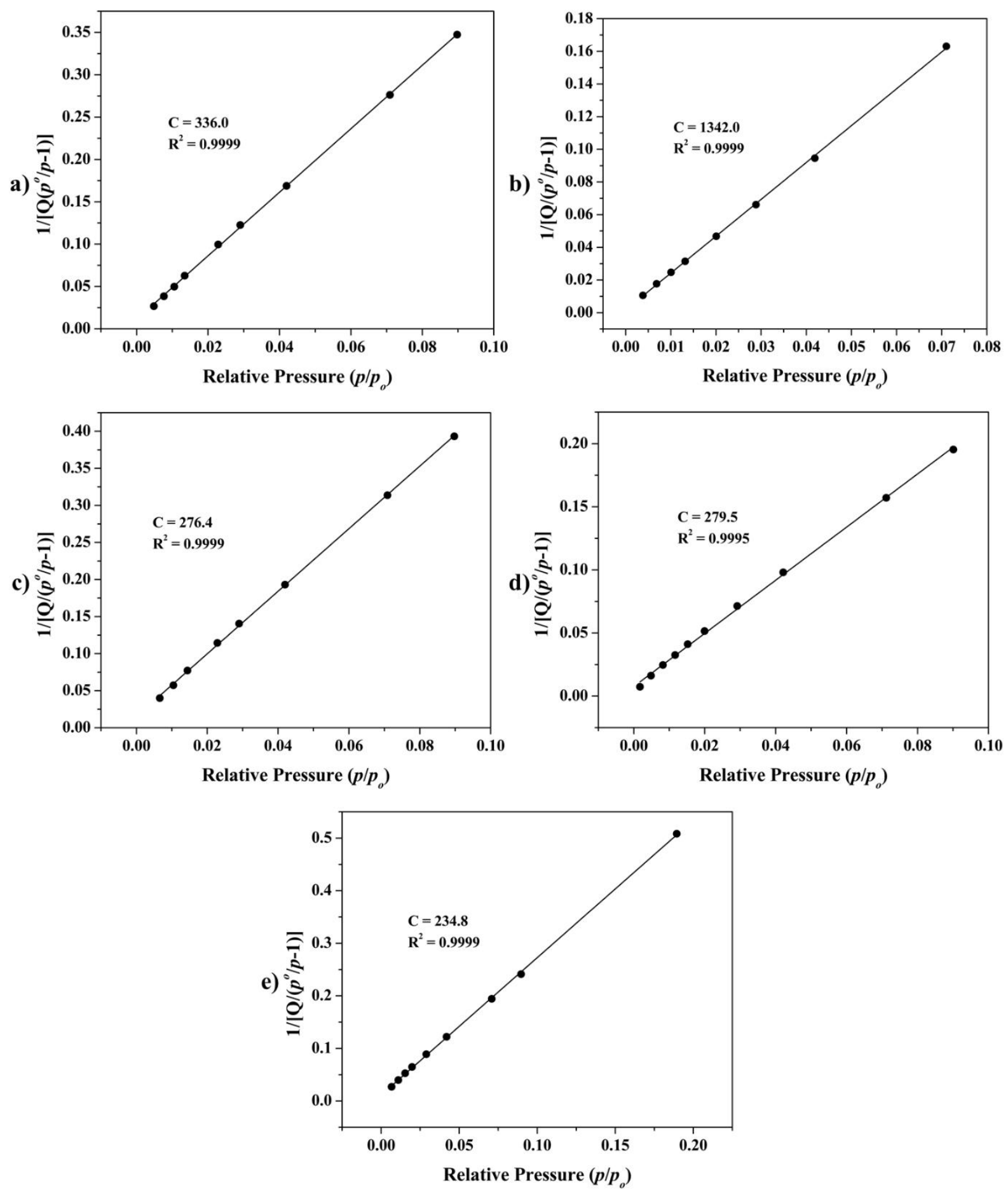

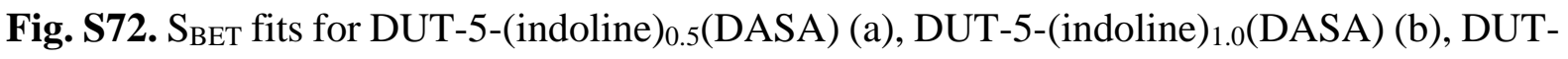

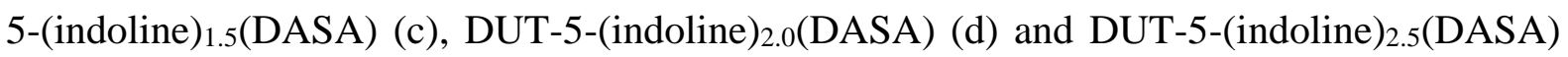
(e). 
Table S6. Pore space dimensions for DUT-5 and modified materials.

\begin{tabular}{|c|c|c|c|}
\hline & $\mathrm{S}_{\mathrm{BET}}\left(\mathrm{m}^{2} / \mathrm{g}\right)$ & DFT pore size $(\AA)$ & $\mathrm{V}_{\text {meso }} / \mathrm{V}_{\text {total }}$ \\
\hline DUT-5 & $1180.2 \pm 3.3$ & $12.0 \& 16.0$ & 0.02 \\
\hline DUT-5(indoline) $)_{0.5}$ & $1627.6 \pm 7.3$ & $12.4 \& 15.6$ & 0.06 \\
\hline DUT-5(indoline) $)_{1.0}$ & $1403.4 \pm 8.5$ & $12.0 \& 15.0$ & 0.03 \\
\hline DUT-5(indoline) $)_{1.5}$ & $977.5 \pm 6.5$ & $13.0 \& 16.0$ & 0.08 \\
\hline DUT-5(indoline) $)_{2.0}$ & $375.6 \pm 0.4$ & $13.0 \& 15.0$ & 0.48 \\
\hline DUT-5(indoline) $)_{2.5}$ & $202.6 \pm 0.9$ & $13.0 \& 15.0$ & 0.73 \\
\hline DUT-5(indoline) $)_{0.5}($ DASA $)$ & $25.9 \pm 0.2$ & 15.2 & 0.90 \\
\hline DUT-5(indoline) $)_{1.0}($ DASA $)$ & $43.2 \pm 0.3$ & - & 0.44 \\
\hline DUT-5(indoline) ${ }_{1.5}($ DASA $)$ & $23.0 \pm 0.2$ & $15.6 \& 18.8$ & 0.76 \\
\hline DUT-5(indoline) $)_{2.0}($ DASA $)$ & $46.2 \pm 0.5$ & $15.2 \& 18.8$ & 0.84 \\
\hline DUT-5(indoline) ${ }_{2.5}($ DASA $)$ & $37.2 \pm 0.2$ & $15.2 \& 18.8$ & 0.89 \\
\hline
\end{tabular}

$\mathrm{N}_{2}$ physisorption isotherms at $77 \mathrm{~K}$ (Fig. S69) provided evidence of incremental defect generation in the DUT-5(indoline) $0.5-2.5$ series based on the appearance and increase in the mesoporosity. The Type I isotherms suggested that DUT-5(indoline) $0.5-1.5$ had negligible mesoporosity. ${ }^{12}$ A change in the isothermal distribution to Type II for DUT-5(indoline) 2.0-2.5 suggested increased mesoporosity which was correlated to a higher indoline stoichiometry. Non-local density functional theory (NLDFT) pore size distributions confirmed the presence of 12 and $15 \AA$ pores with higher stoichiometries of indoline also resulting in mesopores $>100$ $\AA$ (Fig. S69). The micropores detected in DUT-5(indoline) $)_{0.5-2.5}$ are not only consistent with experimental data for the parent MOF, but also with past measurements in the literature. $6,7,13$ Trends derived from isothermal data were also evident in the $\mathrm{S}_{\mathrm{BET}}$ values and mesopore ratios (Table S6). The $\mathrm{S}_{\mathrm{BET}}$ values were consistent with the literature, with an initial increase in surface area compared to the parent MOF, up to $1627.6 \mathrm{~m}^{2} / \mathrm{g}$ (DUT-5(indoline) 1.0 ), before a continuous decrease to $202.6 \mathrm{~m}^{2} / \mathrm{g}$ (DUT-5(indoline) 2.5 ). ${ }^{8,9}$ Mesopore ratios were negligible initially for the parent MOF up to DUT-5(indoline) 1.5 , compared to DUT-5(indoline) $2.0-2.5$ which had ratios of 0.48 and 0.73 , respectively.

The Type II behavior in the $\mathrm{N}_{2}$ adsorption isotherms for DUT-5(indoline) 2.0-2.5 was more prominent in the DUT-5(indoline) 0.5-2.5(DASA) MOFs as substantiated by H4 hysteresis (Fig. S71). ${ }^{12}$ Furthermore, $S_{\text {BETS }}$ were drastically reduced following PSM $\left(25.9-46.2 \mathrm{~m}^{2} / \mathrm{g}\right)$. 
substantially low surface areas are not unheard of for photo-switching MOFs. The $\left[\mathrm{Zn}_{4}(\text { tbazip })_{3}(\mathrm{bpe})_{2}(\mathrm{OH})_{2}\right]$ bpe $\mathrm{MOF}$ is an example of this where the azobenzene containing framework had a surface area of approximately $30 \mathrm{~m}^{2} / \mathrm{g} .{ }^{14}$ Additionally, calculated pore size distributions indicated that mesopore volumes (for pores $>100 \AA$ ) were increased for all members of the series compared to their respective 15 and $18 \AA$ pore volumes (Table S6).

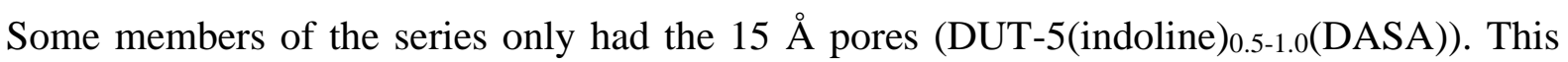

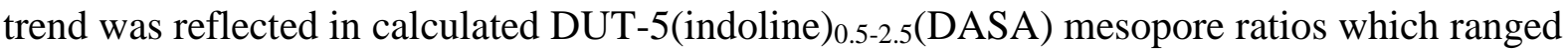
from $0.44-0.90$. The increased mesopore ratios compared to DUT-5(indoline) $0.5-2.5$ are likely due, in part, to the reduction in the crystallinity associated with PSM. These factors effectively resulted in an overall reduction and increase in micropore and mesopore volumes in the DUT5(indoline) 0.5-2.5(DASA) series, respectively. 
S10. $\mathrm{CO}_{2}$ Physisorption ( $\left.298 \mathrm{~K}\right)$

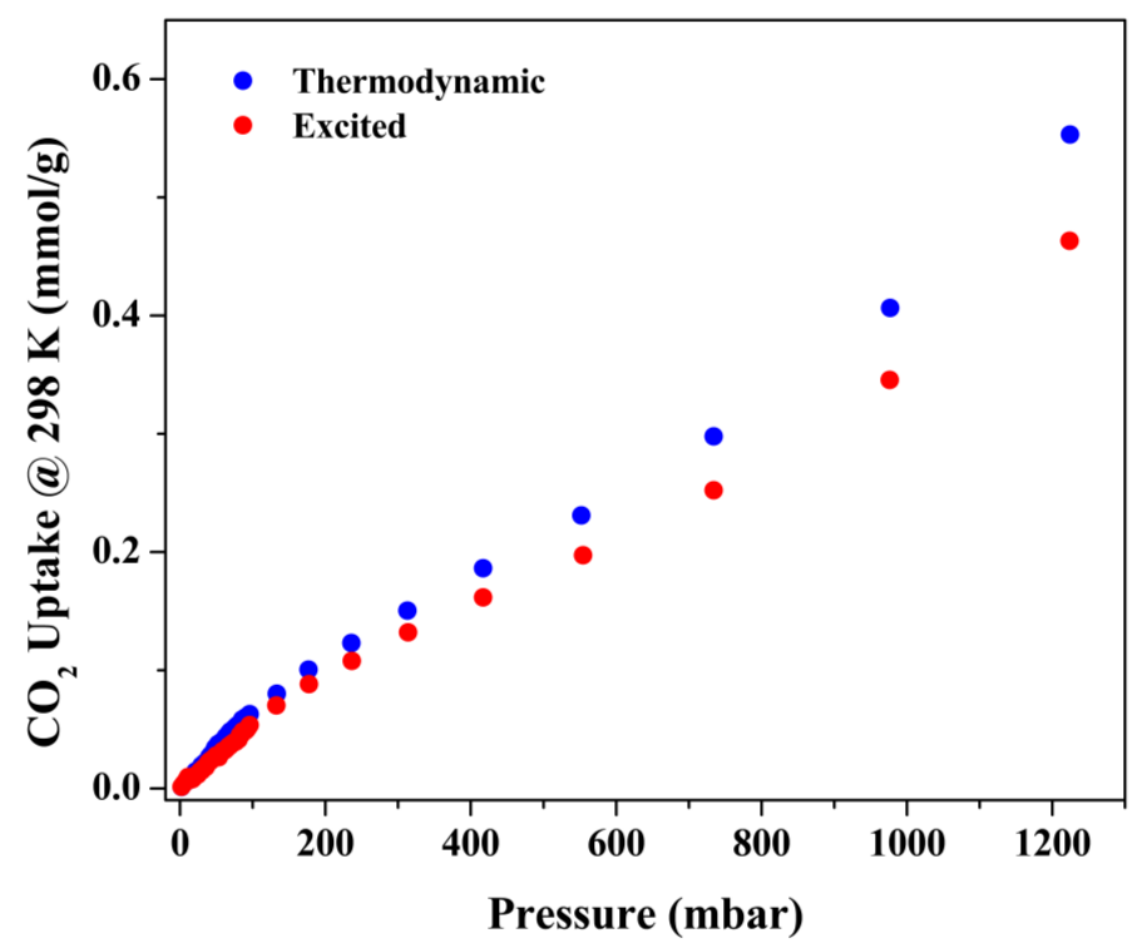

Fig. S73. $\mathrm{CO}_{2}$ physisorption isotherms $\left(298 \mathrm{~K}\right.$ ) for DUT-5(indoline) ${ }_{0.5}(\mathrm{DASA})$, where uptake decreased following irradiation.

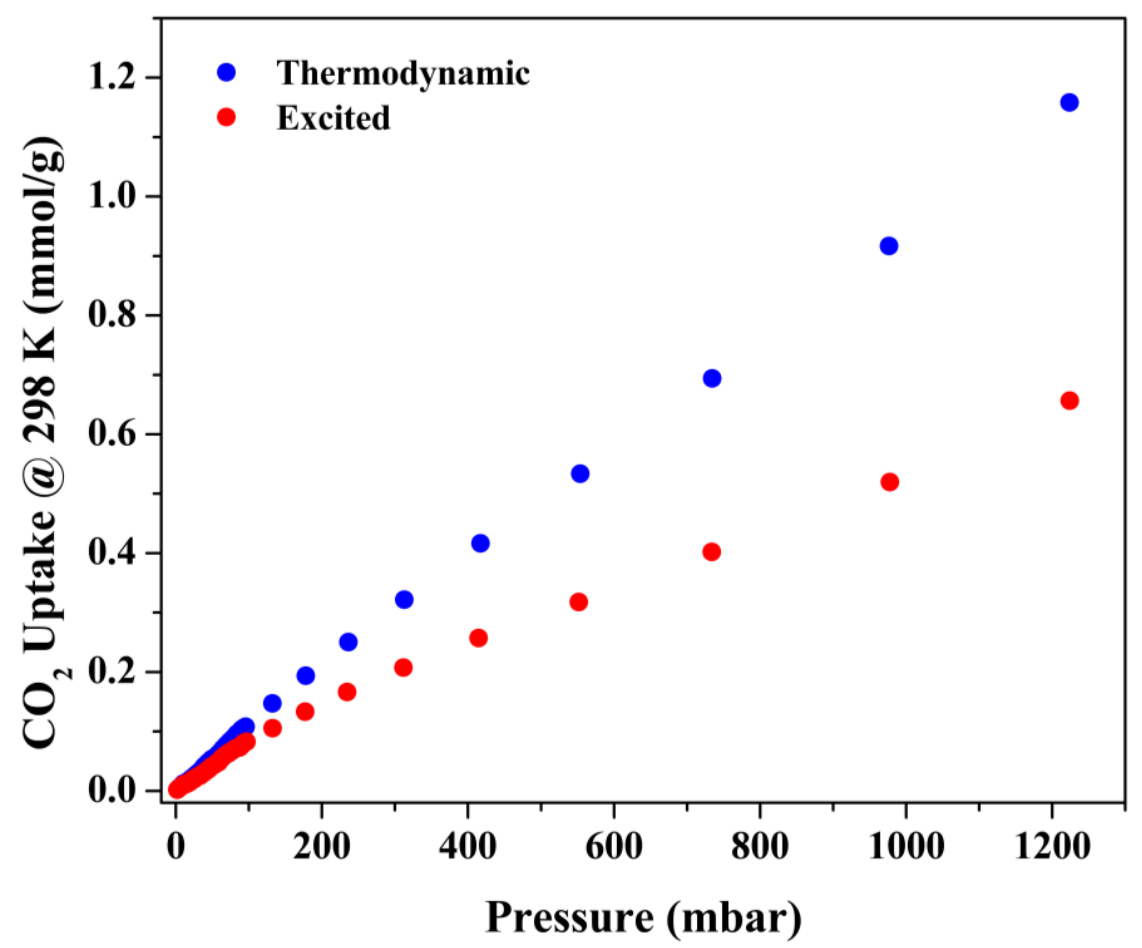

Fig. S74. $\mathrm{CO}_{2}$ physisorption isotherms $(298 \mathrm{~K} \text { ) for DUT-5(indoline })_{1.0}(\mathrm{DASA})$, where uptake decreased following irradiation. 


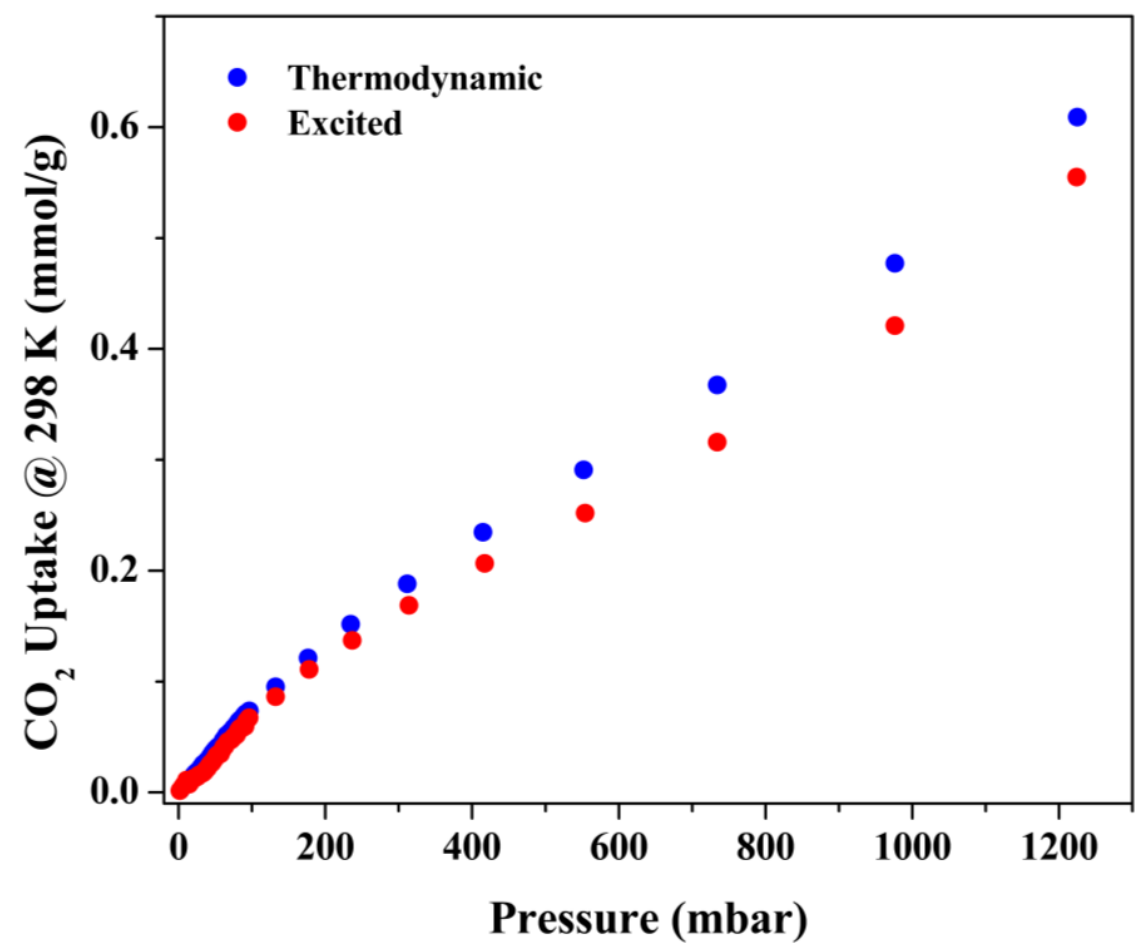

Fig. S75. $\mathrm{CO}_{2}$ physisorption isotherms $(298 \mathrm{~K})$ for DUT-5(indoline) $)_{1.5}(\mathrm{DASA})$, where uptake decreased following irradiation.

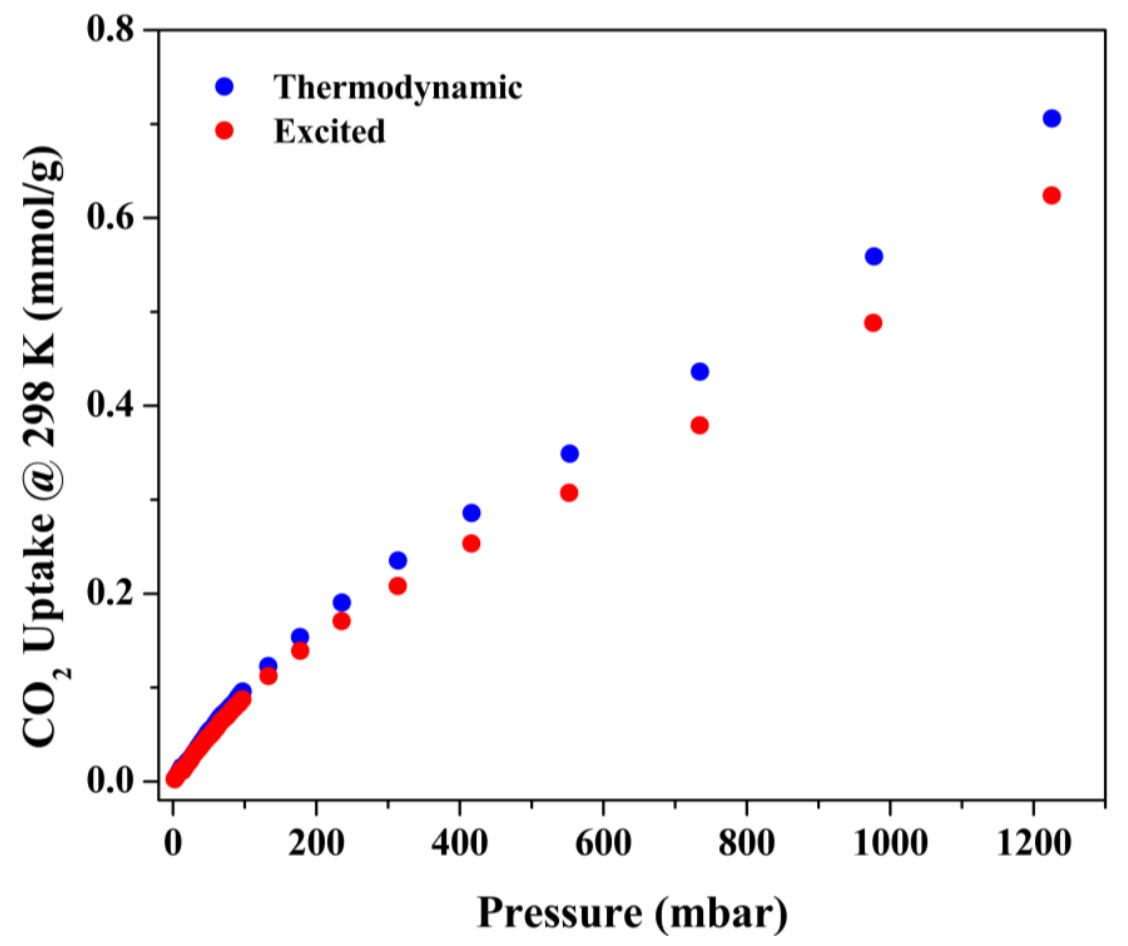

Fig. S76. $\mathrm{CO}_{2}$ physisorption isotherms $(298 \mathrm{~K})$ for DUT-5(indoline $)_{2.0}(\mathrm{DASA})$, where uptake decreased following irradiation. 


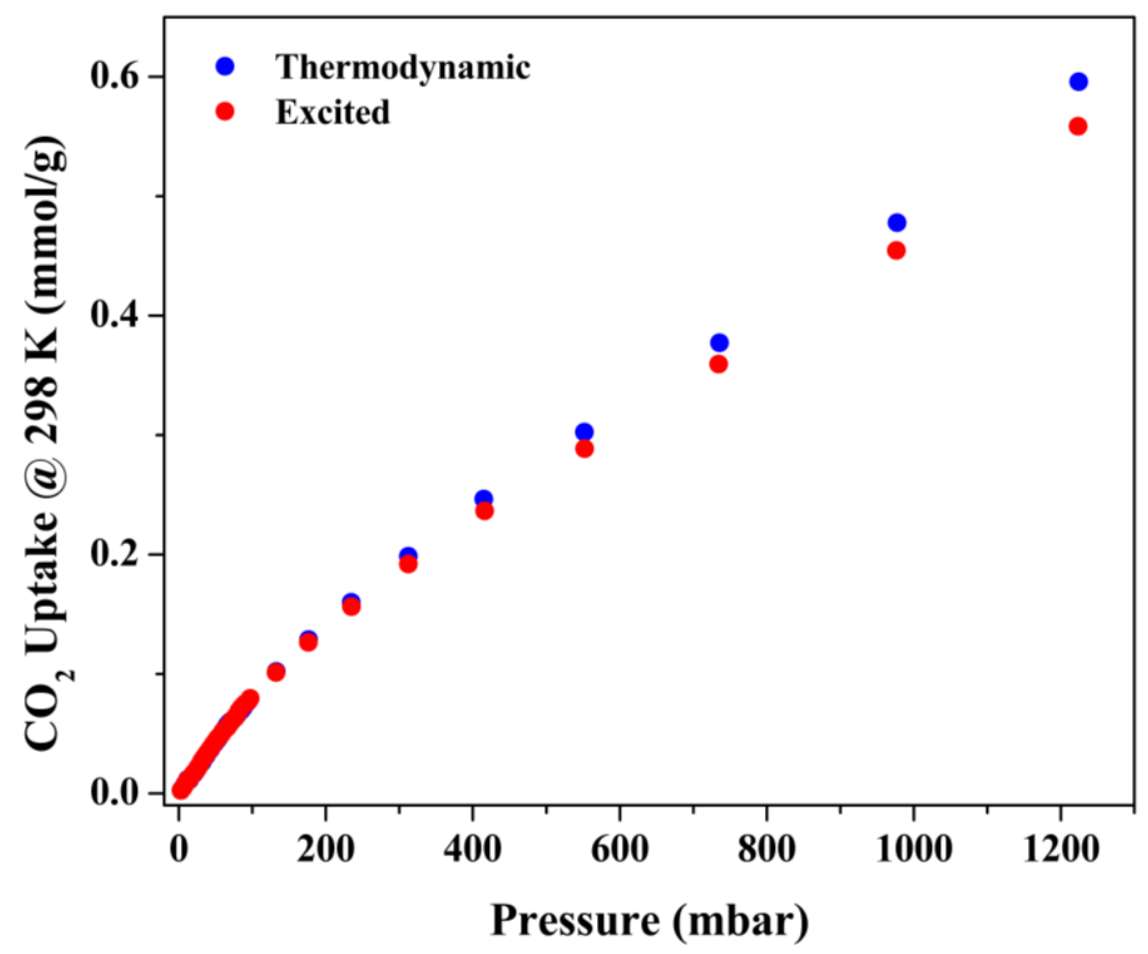

Fig. S77. $\mathrm{CO}_{2}$ physisorption isotherms $(298 \mathrm{~K})$ for DUT-5(indoline) ${ }_{2.5}(\mathrm{DASA})$, where uptake decreased following irradiation.

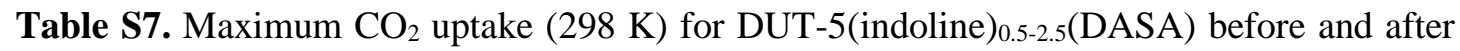
white light irradiation.

\begin{tabular}{lcc}
\hline & Thermodynamic Uptake $(\mathrm{mmol} / \mathrm{g})$ & Excited Uptake $(\mathrm{mmol} / \mathrm{g})$ \\
\hline DUT-5(indoline) $)_{0.5}(\mathrm{DASA})$ & 0.553 & 0.463 \\
DUT-5(indoline) $)_{1.0}(\mathrm{DASA})$ & 1.16 & 0.657 \\
DUT-5(indoline) $)_{1.5}(\mathrm{DASA})$ & 0.609 & 0.555 \\
DUT-5(indoline) $)_{2.0}(\mathrm{DASA})$ & 0.706 & 0.624 \\
DUT-5(indoline) & 0.596 & 0.559 \\
\hline
\end{tabular}




\section{S11. Other Photo-Switching Systems}

Table S8. Relaxation behaviors for photo-switching MOFs and DASA-modified systems.

\begin{tabular}{|c|c|c|}
\hline Compound & Reversion Behavior & Reference \\
\hline$\left[\mathrm{Co}_{2}(\mathrm{NDC})_{2}(\mathrm{AzBIPY})\right]$ & Relaxation over 15 minutes. & 15 \\
\hline $\begin{array}{c}{\left[\mathrm{Cu}_{2}(\mathrm{AzoBPDC})_{2}(\mathrm{AzoBiPyB})\right]} \\
\text { SURMOF }\end{array}$ & Relaxation in < five minutes under visible light. & 16 \\
\hline$\left[\mathrm{Cu}_{2}(\mathrm{BDC})_{2}(\mathrm{AzoBiPyB})\right]$ & Relaxation over 90 minutes. & 17 \\
\hline$\left[\mathrm{Cu}_{2}(\mathrm{DMTPDC})_{2}(\mathrm{AzoBiPyB})\right]$ & Relaxation over 90 minutes. & 17 \\
\hline$\left[\mathrm{Cu}_{2}\left(\mathrm{~F}_{2} \mathrm{AzoBDC}\right)_{2}(\right.$ dabco $\left.)\right]$ SURMOF & Relaxation over three days. & 18 \\
\hline$\left[\mathrm{Cu}_{2}(\mathrm{NDC})_{2}(\mathrm{AzBIPY})\right]$ & Relaxation over 15 minutes. & 15 \\
\hline$\left[\mathrm{Cu}_{2}(\mathrm{SP}-\mathrm{BPDC})_{2}(\right.$ dabco $\left.)\right]$ SURMOF & Relaxation over $c a$. one hour. & 19 \\
\hline$[\mathrm{Zn}(\operatorname{Im})(\mathrm{aIm})]$ & Relaxation over one hour. & 20 \\
\hline$\left[\mathrm{Zn}_{2}(\mathrm{BPDC})_{2}(\mathrm{BPMTC})\right]$ & Relaxation in $<$ three minutes under visible light. & 21 \\
\hline$\left[\mathrm{Zn}_{2}(\mathrm{DBTD})(\mathrm{TNDS})\right]$ & Relaxation in $<$ three minutes under visible light. & 21 \\
\hline$\left[\mathrm{Zn}_{2}(\mathrm{SDC})_{2}(\mathrm{BPMTC})\right]$ & Relaxation in $<$ three minutes under visible light. & 21 \\
\hline$\left[\mathrm{Zn}_{4}(\text { tbazip })_{3}(\text { bpe })_{2}(\mathrm{OH})_{2}\right] \cdot$ bpe & Relaxation over two hours. & 14 \\
\hline CAU-5 & Relaxation over 15 minutes. & 22 \\
\hline Cr-MIL-101_amide & Relaxation over 15 minutes. & 23 \\
\hline Cr-MIL-101_urea & Relaxation over 15 minutes. & 23 \\
\hline DASA polymer & Relaxation in PhMe over 90 minutes in the dark. & 24 \\
\hline DASA/SP & $\begin{array}{l}\text { Relaxation in DCM over } 20 \text { minutes in the dark. } \\
\text { Spin coated films required heating at } 60^{\circ} \mathrm{C} \text {. }\end{array}$ & 25 \\
\hline DASA-modified $\mathrm{Fe}_{3} \mathrm{O}_{4}$ nanoparticles & Irreversible. & 26 \\
\hline DASA/HPCC18NP mixtures & Excited state stable over several months. & 27 \\
\hline $\begin{array}{l}\text { DASA-modified poly(glycidyl } \\
\text { methacrylate-co-dimethylacrylamide) }\end{array}$ & Partial relaxation in $\mathrm{CHCl}_{3}$. & 28 \\
\hline DASA-modified polymer dots & Reversion with acid. & 29 \\
\hline DMOF & Relaxation in $<$ one minute. & 30 \\
\hline F-azo-MIL-53(Al) & Relaxation over 20 hours. & 31 \\
\hline
\end{tabular}




\begin{tabular}{|c|c|c|}
\hline Compound & Reversion Behavior & Reference \\
\hline F-azo-UiO-66-(Zr) & Relaxation over 20 hours. & 31 \\
\hline $\begin{array}{l}\text { HKUST-1 films with azobenzene } \\
\text { guests }\end{array}$ & Relaxation over 20 minutes. & 32 \\
\hline $\begin{array}{l}\text { Hydroxyl-terminated polybutadiene } \\
\text { with embedded DASA }\end{array}$ & Relaxation over 30 minutes at $120^{\circ} \mathrm{C}$. & 33 \\
\hline JUC-120 (MOF) & Thermal relaxation over seven days. & 34 \\
\hline MELD polymersome & Relaxation over two hours. & 35 \\
\hline PYRA polymersome & Relaxation over 30 minutes. & 35 \\
\hline SN-DASA-POM-DASA & Irreversible (DASA switching only). & 36 \\
\hline SP-1 $@$ @MIL-53(Al) & Required heating. & 37 \\
\hline SP-1x@MIL-68(Ga) & Required heating. & 37 \\
\hline SP-1, @MIL-68(In) & Required heating. & 37 \\
\hline SP-1 @ @MOF-5 & Required heating. & 37 \\
\hline SP-O@MIL-53(Al) & Relaxation in $<$ one minute. & 38 \\
\hline SP-O@MIL-68(Ga) & Relaxation in $<$ one minute. & 38 \\
\hline SP-O@MIL-68(In) & Relaxation in $<$ one minute. & 38 \\
\hline SP-O@MOF-5 & Relaxation in $<$ one minute. & 38 \\
\hline SSP@ZIF-8 & Relaxation in $<$ five minutes. & 39 \\
\hline TBA-DASA-POM-DASA & Irreversible. & 36 \\
\hline UBMOF-1 & $\begin{array}{l}\text { Partial relaxation over two hours under visible } \\
\text { light. }\end{array}$ & 40 \\
\hline
\end{tabular}

NB. This is not an exhaustive list of compounds.

A short summary of the reversion stimuli for select photo-switching MOFs and DASAmodified systems are detailed in Table S8. Photo-switching MOFs detailed are limited to those with structural responsive organic functional groups (specifically, azobenzenes, diarylethenes, spiropyrans and spirooxazines). Select MOFs, such as the frameworks with embedded spiropyrans described by Ruschewitz and co-workers, ${ }^{37}$ require heating for reversion to the thermodynamic state. Other MOFs with contrasting behaviour respond to either an alternative light source or under dark conditions. F-azo-MIL-53(Al), ${ }^{31} \quad$ F-azo-UiO-66-(Zr), ${ }^{31}$ $\left[\mathrm{Cu}_{2}\left(\mathrm{~F}_{2} \mathrm{AzoBDC}\right)_{2}(\mathrm{dabco})\right]^{18}$ and JUC-120 ${ }^{34}$ demonstrate substantial stability in their excited 
states with reversion ranging from 20 hours to seven days, significantly less than that observed for the DUT-5(indoline) $0.5-2.5$ (DASA) series. The bistability exhibited by DUT-5(indoline) 0.5 2.5(DASA) is most closely consistent with the DASA/HPCC18NP mixtures which were also stable in their excited state for several months. ${ }^{27}$ 


\section{References}

(1) Toby, B. H.; Von Dreele, R. B. GSAS-II : The Genesis of a Modern Open-Source All Purpose Crystallography Software Package . J. Appl. Crystallogr. 2013, 46 (2), 544549.

(2) Fulmer, G. R.; Miller, A. J. M.; Sherden, N. H.; Gottlieb, H. E.; Nudelman, A.; Stoltz, B. M.; Bercaw, J. E.; Goldberg, K. I. NMR Chemical Shifts of Trace Impurities: Common Laboratory Solvents, Organics, and Gases in Deuterated Solvents Relevant to the Organometallic Chemist. Organometallics 2010, 29 (9), 2176-2179.

(3) Brunauer, S.; Emmett, P. H.; Teller, E. Adsorption of Gases in Multimolecular Layers. J. Am. Chem. Soc. 1938, 60 (2), 309-319.

(4) Helmy, S.; Oh, S.; Leibfarth, F. A.; Hawker, C. J.; Read De Alaniz, J. Design and Synthesis of Donor-Acceptor Stenhouse Adducts: A Visible Light Photoswitch Derived from Furfural. J. Org. Chem. 2014, 79 (23), 11316-11329.

(5) Helmy, S.; Leibfarth, F.; Oh, S.; Poelma, J.; Hawker, C. J.; Read De Alaniz, J. Photoswitching Using Visible Light: A New Class of Organic Photochromic Molecules. J. Am. Chem. Soc. 2014, 136 (23), 8169-8172.

(6) Senkovska, I.; Hoffmann, F.; Fröba, M.; Getzschmann, J.; Böhlmann, W.; Kaskel, S. New Highly Porous Aluminium Based Metal-Organic Frameworks: $\mathrm{Al}(\mathrm{OH})(\mathrm{Ndc})(\mathrm{Ndc}$ $=$ 2,6-Naphthalene Dicarboxylate) and $\mathrm{Al}(\mathrm{OH})(\mathrm{Bpdc}) \quad\left(\mathrm{Bpdc}=4,4^{\prime}\right.$-Biphenyl Dicarboxylate). Microporous Mesoporous Mater. 2009, 122 (1-3), 93-98.

(7) Couck, S.; Liu, Y. Y.; Leus, K.; Baron, G. V.; Van Der Voort, P.; Denayer, J. F. M. Gas Phase Adsorption of Alkanes, Alkenes and Aromatics on the Sulfone-DUT-5 Metal Organic Framework. Microporous Mesoporous Mater. 2015, 206 (C), 217-225.

(8) Liang, W.; Li, L.; Hou, J.; Shepherd, N. D.; Bennett, T. D.; D’Alessandro, D. M.; Chen, V. Linking Defects, Hierarchical Porosity Generation and Desalination Performance in Metal-Organic Frameworks. Chem. Sci. 2018, 9 (14), 3508-3516.

(9) Liang, W.; Coghlan, C. J.; Ragon, F.; Rubio-Martinez, M.; D’Alessandro, D. M.; Babarao, R. Defect Engineering of UiO-66 for CO2and H2O Uptake - A Combined Experimental and Simulation Study. Dalt. Trans. 2016, 45 (11), 4496-4500. 
(10) Shearer, G. C.; Chavan, S.; Bordiga, S.; Svelle, S.; Olsbye, U.; Lillerud, K. P. Defect Engineering: Tuning the Porosity and Composition of the Metal-Organic Framework UiO-66 via Modulated Synthesis. Chem. Mater. 2016, 28 (11), 3749-3761.

(11) Ren, J.; Ledwaba, M.; Musyoka, N. M.; Langmi, H. W.; Mathe, M.; Liao, S.; Pang, W. Structural Defects in Metal-Organic Frameworks (MOFs): Formation, Detection and Control towards Practices of Interests. Coord. Chem. Rev. 2017, 349, 169-197.

(12) Thommes, M.; Kaneko, K.; Neimark, A. V.; Olivier, J. P.; Rodriguez-Reinoso, F.; Rouquerol, J.; Sing, K. S. W. Physisorption of Gases, with Special Reference to the Evaluation of Surface Area and Pore Size Distribution (IUPAC Technical Report). Pure Appl. Chem. 2015, 87 (9-10), 1051-1069.

(13) Gotthardt, M. A.; Grosjean, S.; Brunner, T. S.; Kotzel, J.; Gänzler, A. M.; Wolf, S.; Bräse, S.; Kleist, W. Synthesis and Post-Synthetic Modification of Amine-, Alkyne-, Azide- and Nitro-Functionalized Metal-Organic Frameworks Based on DUT-5. Dalt. Trans. 2015, 44 (38), 16802-16809.

(14) Caddy, J. S.; Faust, T. B.; Walton, I. M.; Cox, J. M.; Benedict, J. B.; Solomon, M. B.; Southon, P. D.; Kepert, C. J.; D’Alessandro, D. M. Photoactive and Physical Properties of an Azobenzene-Containing Coordination Framework. Aust. J. Chem. 2017, 70 (11), $1171-1179$.

(15) Modrow, A.; Feyand, M.; Zargarani, D.; Herges, R.; Stock, N. Systematic Investigation of Porous Inorganic-Organic Hybrid Compounds with Photo-Switchable Properties. Zeitschrift fur Anorg. und Allg. Chemie 2012, 638 (12-13), 2138-2143.

(16) Wang, Z.; Knebel, A.; Grosjean, S.; Wagner, D.; Bräse, S.; Wöll, C.; Caro, J.; Heinke, L. Tunable Molecular Separation by Nanoporous Membranes. Nat. Commun. 2016, 7, 13872 .

(17) Yu, X.; Wang, Z.; Buchholz, M.; Füllgrabe, N.; Grosjean, S.; Bebensee, F.; Bräse, S.; Wöll, C.; Heinke, L. Cis-to-Trans Isomerization of Azobenzene Investigated by Using Thin Films of Metal-Organic Frameworks. Phys. Chem. Chem. Phys. 2015, 17 (35), $22721-22725$.

(18) Müller, K.; Knebel, A.; Zhao, F.; Bléger, D.; Caro, J.; Heinke, L. Switching Thin Films of Azobenzene-Containing Metal-Organic Frameworks with Visible Light. Chem. - A 
Eur. J. 2017, 23 (23), 5434-5438.

(19) Kanj, A. B.; Chandresh, A.; Gerwien, A.; Grosjean, S.; Bräse, S.; Wang, Y.; Dube, H.; Heinke, L. Proton-Conduction Photomodulation in Spiropyran-Functionalized MOFs with Large on-off Ratio. Chem. Sci. 2020, 11 (5), 1404-1410.

(20) Bernt, S.; Feyand, M.; Modrow, A.; Wack, J.; Senker, J.; Stock, N. A Mixed-Linker ZIF Containing a Photoswitchable Phenylazo Group. Eur. J. Inorg. Chem. 2011, No. 35, $5378-5383$.

(21) Dolgopolova, E. A.; Galitskiy, V. A.; Martin, C. R.; Gregory, H. N.; Yarbrough, B. J.; Rice, A. M.; Berseneva, A. A.; Ejegbavwo, O. A.; Stephenson, K. S.; Kittikhunnatham, P.; et al. Connecting Wires: Photoinduced Electronic Structure Modulation in MetalOrganic Frameworks. J. Am. Chem. Soc. 2019, 141 (13), 5350-5358.

(22) Modrow, A.; Zargarani, D.; Herges, R.; Stock, N. The First Porous MOF with Photoswitchable Linker Molecules. Dalt. Trans. 2011, 40 (16), 4217-4222.

(23) Modrow, A.; Zargarani, D.; Herges, R.; Stock, N. Introducing a Photo-Switchable AzoFunctionality inside Cr-MIL-101-NH2by Covalent Post-Synthetic Modification. Dalt. Trans. 2012, 41 (28), 8690-8696.

(24) Sinawang, G.; Wu, B.; Wang, J.; Li, S.; He, Y. Polystyrene Based Visible Light Responsive Polymer with Donor-Acceptor Stenhouse Adduct Pendants. Macromol. Chem. Phys. 2016, 217 (21), 2409-2414.

(25) Mostafavi, S. H.; Li, W.; Clark, K. D.; Stricker, F.; Alaniz, J. R. De; Bardeen, C. J. Photoinduced Deadhesion of a Polymer Film Using a Photochromic Donor-Acceptor Stenhouse Adduct. Macromolecules 2019, 52, 6311-6317.

(26) Ahrens, J.; Bian, T.; Vexler, T.; Klajn, R. Irreversible Bleaching of Donor-Acceptor Stenhouse Adducts on the Surfaces of Magnetite Nanoparticles. ChemPhotoChem 2017, 1 (5), 230-236.

(27) Nau, M.; Seelinger, D.; Biesalski, M. Independent Two Way Switching of the Wetting Behavior of Cellulose-Derived Nanoparticle Surface Coatings by Light and by Temperature. Adv. Mater. Interfaces 2019, 6 (17), 1900378.

(28) Balamurugan, A.; Lee, H. A Visible Light Responsive On-Off Polymeric Photoswitch 
for the Colorimetric Detection of Nerve Agent Mimics in Solution and in the Vapor Phase. Macromolecules 2016, 49 (7), 2568-2574.

(29) Zhong, D.; Cao, Z.; Wu, B.; Zhang, Q.; Wang, G. Polymer Dots of DASAFunctionalized Polyethyleneimine: Synthesis, Visible Light/PH Responsiveness, and Their Applications as Chemosensors. Sensors Actuators, B Chem. 2018, 254, 385-392.

(30) Luo, F.; Fan, C. Bin; Luo, M. B.; Wu, X. L.; Zhu, Y.; Pu, S. Z.; Xu, W. Y.; Guo, G. C. Photoswitching CO2capture and Release in a Photochromic Diarylethene MetalOrganic Framework. Angew. Chemie - Int. Ed. 2014, 53 (35), 9298-9301.

(31) Castellanos, S.; Goulet-Hanssens, A.; Zhao, F.; Dikhtiarenko, A.; Pustovarenko, A.; Hecht, S.; Gascon, J.; Kapteijn, F.; Bléger, D. Structural Effects in Visible-LightResponsive Metal-Organic Frameworks Incorporating Ortho -Fluoroazobenzenes. Chem. - A Eur. J. 2016, 22 (2), 746-752. https://doi.org/10.1002/chem.201503503.

(32) Fu, W. Q.; Liu, M.; Gu, Z. G.; Chen, S. M.; Zhang, J. Liquid Phase Epitaxial Growth and Optical Properties of Photochromic Guest-Encapsulated MOF Thin Film. Cryst. Growth Des. 2016, 16 (9), 5487-5492.

(33) Mason, B. P.; Whittaker, M.; Hemmer, J.; Arora, S.; Harper, A.; Alnemrat, S.; McEachen, A.; Helmy, S.; Read De Alaniz, J.; Hooper, J. P. A Temperature-Mapping Molecular Sensor for Polyurethane-Based Elastomers. Appl. Phys. Lett. 2016, 108 (4), 041906/1-041906/4.

(34) Zhang, F.; Zou, X.; Feng, W.; Zhao, X.; Jing, X.; Sun, F.; Ren, H.; Zhu, G. MicrowaveAssisted Crystallization Inclusion of Spiropyran Molecules in Indium Trimesate Films with Antidromic Reversible Photochromism. J. Mater. Chem. 2012, 22 (48), 2501925026.

(35) Rifaie-Graham, O.; Ulrich, S.; Galensowske, N. F. B.; Balog, S.; Chami, M.; Rentsch, D.; Hemmer, J. R.; Read De Alaniz, J.; Boesel, L. F.; Bruns, N. Wavelength-Selective Light-Responsive DASA-Functionalized Polymersome Nanoreactors. J. Am. Chem. Soc. 2018, 140 (25), 8027-8036.

(36) Boulmier, A.; Haouas, M.; Tomane, S.; Michely, L.; Dolbecq, A.; Vallée, A.; Brezová, V.; Versace, D. L.; Mialane, P.; Oms, O. Photoactive Polyoxometalate/DASA Covalent Hybrids for Photopolymerization in the Visible Range. Chem. - A Eur. J. 2019, 25 (63), 
$14349-14357$.

(37) Schwartz, H. A.; Olthof, S.; Schaniel, D.; Meerholz, K.; Ruschewitz, U. Solution-Like Behavior of Photoswitchable Spiropyrans Embedded in Metal-Organic Frameworks. Inorg. Chem. 2017, 56 (21), 13100-13110.

(38) Schwartz, H. A.; Werker, M.; Tobeck, C.; Christoffels, R.; Schaniel, D.; Olthof, S.; Meerholz, K.; Kopacka, H.; Huppertz, H.; Ruschewitz, U. Novel Photoactive Spirooxazine Based Switch@MOF Composite Materials. ChemPhotoChem 2020, 195206.

(39) Liang, H. Q.; Guo, Y.; Shi, Y.; Peng, X.; Liang, B.; Chen, B. A Light-Responsive MetalOrganic Framework Hybrid Membrane with High On/Off Photoswitchable Proton Conductivity. Angew. Chemie - Int. Ed. 2020, 1-7.

(40) Patel, D. G.; Walton, I. M.; Cox, J. M.; Gleason, C. J.; Butzer, D. R.; Benedict, J. B. Photoresponsive Porous Materials: The Design and Synthesis of Photochromic Diarylethene-Based Linkers and a Metal-Organic Framework. Chem. Commun. 2014, 50 (20), 2653-2656. 